\title{
The Asymptotic Properties of GMM and Indirect Inference under Second-order Identification
}

PROSPER DOVONON ALASTAIR R. HALL 
2018s-37

\title{
The Asymptotic Properties of GMM and Indirect Inference under Second-order Identification
}

\author{
Prosper Dovonon, Alastair R. Hall
}

\section{Série Scientifique \\ Scientific Series}

\author{
Montréal \\ Décembre/Decembre 2018
}

(C) 2018 Prosper Dovonon, Alastair R. Hall. Tous droits réservés. All rights reserved. Reproduction partielle permise avec citation du document source, incluant la notice C. Short sections may be quoted without explicit permission, if full credit, including (C) notice, is given to the source.

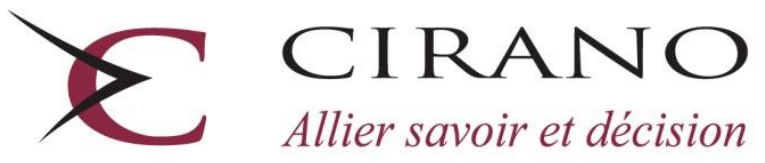

Centre interuniversitaire de recherche en analyse des organisations 


\section{CIRANO}

Le CIRANO est un organisme sans but lucratif constitué en vertu de la Loi des compagnies du Québec. Le financement de son infrastructure et de ses activités de recherche provient des cotisations de ses organisations-membres, d'une subvention d'infrastructure du gouvernement du Québec, de même que des subventions et mandats obtenus par ses équipes de recherche.

CIRANO is a private non-profit organization incorporated under the Quebec Companies Act. Its infrastructure and research activities are funded through fees paid by member organizations, an infrastructure grant from the government of Quebec, and grants and research mandates obtained by its research teams.

\section{Les partenaires du CIRANO}

\section{Partenaires corporatifs}

Autorité des marchés financiers

Banque de développement du Canada

Banque du Canada

Banque Laurentienne du Canada

Banque Nationale du Canada

Bell Canada

BMO Groupe financier

Caisse de dépôt et placement du Québec

Énergir

Hydro-Québec

Innovation, Sciences et Développement économique Canada

Intact Corporation Financière

Investissements PSP

Ministère de l'Économie, de la Science et de l'Innovation

Ministère des Finances du Québec

Mouvement Desjardins

Power Corporation du Canada

Rio Tinto

Ville de Montréal

\section{Partenaires universitaires}

École de technologie supérieure

HEC Montréal

Institut national de la recherche scientifique

Polytechnique Montréal

Université Concordia

Université de Montréal

Université de Sherbrooke

Université du Québec

Université du Québec à Montréal

Université Laval

Université McGill

Le CIRANO collabore avec de nombreux centres et chaires de recherche universitaires dont on peut consulter la liste sur son site web.

Les cahiers de la série scientifique (CS) visent à rendre accessibles des résultats de recherche effectuée au CIRANO afin de susciter échanges et commentaires. Ces cahiers sont écrits dans le style des publications scientifiques. Les idées et les opinions émises sont sous l'unique responsabilité des auteurs et ne représentent pas nécessairement les positions du CIRANO ou de ses partenaires.

This paper presents research carried out at CIRANO and aims at encouraging discussion and comment. The observations and viewpoints expressed are the sole responsibility of the authors. They do not necessarily represent positions of CIRANO or its partners.

ISSN 2292-0838 (en ligne) 


\title{
The Asymptotic Properties of GMM and Indirect Inference under Second-order Identification*
}

\author{
Prosper Dovonon ${ }^{\dagger}$, Alastair R. Hall ${ }^{\ddagger}$
}

\begin{abstract}
This paper presents a limiting distribution theory for GMM and Indirect Inference estimators when local identification conditions fail at first-order but hold at second-order. These limit distributions are shown to be non-standard, but we show that they can be easily simulated, making it possible to perform inference about the parameters in this setting. We illustrate our results in the context of a dynamic panel data model in which the parameter of interest is identified locally at second order by non-linear moment restrictions but not at first order at a particular point in the parameter space. Our simulation results indicate that our theory leads to reliable inferences in moderate to large samples in the neighbourhood of this point of first-order identification failure. In contrast, inferences based on standard asymptotic theory (derived under the assumption of first-order local identification) are very misleading in the neighbourhood of the point of firstorder local identification failure.
\end{abstract}

Keywords: Moment-based Estimation, First-order Identification failure, Minimum-chi Squared Estimation, Simulation-based Estimation

\footnotetext{
* The paper has benefited from the comments of the Guest Editors, two referees, and participants at the Conference in honour of Jean-Marie Dufour at Cireq in Montreal (May 2016), a seminar at the University of Nottingham and the Canadian Econometric Study Group meeting in London, Ontario, Canada (October 2016).

${ }^{\dagger}$ Department of Economics, Concordia University, 1455 de Maisonneuve Blvd. West, Montreal, Quebec, H3G 1M8 Canada. E-mail: prosper.dovonon@concordia.ca.

¥ Corresponding author. Economics, School of Social Sciences, University of Manchester, Manchester, M13

9PL, UK. E-mail: alastair.hall@manchester.ac.uk.
} 


\section{Introduction}

Generalized Method of Moments (GMM) was introduced by Lars Hansen in a paper published in Econometrica in 1982. Since then this article has come to be recognized as one of the most influential papers in econometrics. ${ }^{1}$ One aspect of this influence is that applications of GMM have demonstrated the power of thinking in terms of moment conditions in econometric estimation. This, in turn, can be said to have inspired the development of other moment-based approaches in econometrics, a leading example of which is Indirect Inference (II). GMM can be applied in wide variety of situations including those where the distribution of the data is unknown and those where it is known but the likelihood is intractable. In the latter scenario, it was realized in the late 1980's and early 1990's that simulation-based methods provide an alternative - and often more efficient way - to estimate the model parameters than GMM. A number of methods were proposed: Method of Simulated Moments (McFadden, 1989), Simulated Method of Moments (SMM, Duffie and Singleton, 1993), Indirect Inference (II, Gourieroux, Monfort, and Renault, 1993, Smith, 1990, 1993) ${ }^{2}$ and Efficient Method of Moments (EMM, Gallant and Tauchen, 1996). While SMM and EMM have their distinctive elements, both can be viewed as examples of II as they have the "indirect" feature of estimating parameters of the model of interest by matching moments from a different - and potentially misspecified - model.

The standard first-order inference frameworks for Generalized Method of Moments (GMM) and Indirect Inference (II) rest crucially on the assumption of first-order local identification that is, a certain derivative matrix has full rank when evaluated at the true parameter value. However, it has been realized that in a number of situations first-order identification either fails or is close to failing with the result that inferences based on the standard framework are misleading. To date, this concern and its consequences have largely been explored in the context of GMM, but recently concerns about identification have been raised in dynamic stochastic general equilibrium (DSGE) models to which GMM and II have been applied. ${ }^{3}$

Within the GMM framework, these concerns about the consequences of identification have mostly arisen in the special case of Generalized Instrumental Variables (GIV) estimation (Hansen and Singleton, 1982) in which the moment condition derives from the orthogonality of a function $u_{t}(\theta)$, involving the parameter vector $\theta$, to a vector of instruments, $z_{t}$. In this case, the condition for first-order local identification is that $\partial u_{t}(\theta) / \partial \theta$ (evaluated at the true parameter value, $\theta_{0}$ ) has a sufficiently strong relationship to $z_{t}$ in the population. However, if this threshold is only marginally satisfied then the standard first-order asymptotic theory can provide a very poor approximation to the finite sample behaviour of various GMM-based statistics. To help derive more accurate approximations, Staiger and Stock (1997) introduced the concept of weak identification. Statistical analyses demonstrated that key statistics behave very differently under weak identification than under the standard first-order asymptotic framework with its assumption of first-order local identification. ${ }^{4}$ For example, Dufour (1997) demonstrated that the potential presence of weak identification renders the conventional "estimator plus/minus a multiple of the standard error"

\footnotetext{
${ }^{1}$ For example, see The Royal Swedish Academy of Sciences (2013), p.24.

${ }^{2}$ Smith (1993) refers to the method as "simulated quasi-maximum likelihood" and his analysis covers a more restrictive setting than that of Gourieroux, Monfort, and Renault (1993).

${ }^{3}$ Applications of GMM/II to DSGE include Christiano, Eichenbaum, and Evans (2005), Coenen, Levin, and Christoffel (2007), Dupaigne, Fève, and Matheron (2007), Ruge-Murcia (2007), Le, Meenagh, Minford, and Wickens (2011).

${ }^{4}$ For example, Staiger and Stock (1997) and Stock and Wright (2000) derive the properties of various estimators such as GMM in linear and nonlinear models respectively.
} 
confidence interval invalid. In response, the focus shifted to developing inference techniques that are valid irrespective of the quality of the identification, such as Kleibergen's (2005) K-statistic. For our purposes here, it is not necessary to summarize subsequent developments within the weak identification framework; it suffices to note that weak identification involves a situation in which both first-order local identification and global identification fail (in the limit). ${ }^{5}$

Canova and Sala (2009) argue that, at their time of writing, the quality of the identification in DSGE models was often neglected, and also that there are grounds for suspecting identification may fail in certain cases of interest. Iskrev (2010), Komunjer and Ng (2011) and Qu and Tkachenko (2012) derive conditions for first-order local identification using alternative representations of the model. In this context, the responses to potential identification failure have been twofold. The first approach is the same as in the GMM literature and is based on developing inference techniques that are robust to weak identification, for example see Dufour, Khalaf, and Kichian (2013) and $\mathrm{Qu}$ (2014). The second approach views the source of identification failure as deriving from the method used to solve the DSGE for the path of the variables. DSGE models are typically highly nonlinear, and so as a result practitioners have resorted to using approximations in solving the models. For the most part, first-order approximations have been used but Mutschler (2015) has recently demonstrated that these may be the source of identification failures, finding that the use of second-order approximations restores first-order local identification in some cases.

As is evident from the above discussion, the focus of the above analyses is on first-order local identification - understandably, as this condition is crucial for the standard first-order asymptotic framework. In linear models, first-order local and global identification are the same, but in nonlinear models, they are not: local identification can fail at first order but hold at a higher order. Furthermore, in such cases, it is possible to develop a framework for inference based on large sample arguments. For the case where local identification holds at second but not first order, Sargan (1983) and Rotnitzky, Cox, Bottai, and Robins (2000) develop a limiting distribution theory for estimators obtained respectively by IV in a nonlinear in parameters model and Maximum Likelihood (ML). Dovonon and Renault (2009, 2013) derive the limiting distribution of the GMM overidentifying restrictions test statistic. This pattern of identification has been shown to arise in a number of situations in statistics and econometrics such as: ML for skew-normal distributions, e.g. Azzalini (2005); ML for binary response models based on skew-normal distributions, Stingo, Stanghellini, and Capobianco (2011); ML for missing not at random (MNAR) models, e.g. Jansen, Hens, Molenberghs, Aerts, Verbeke, and Kenward (2006); GMM estimation of conditionally heteroscedastic factor models, Dovonon and Renault $(2009,2013)$; GMM estimation of panel data models using second moments, Madsen (2009); ML estimation of panel data models, Kruiniger (2014).

In this paper, we consider the case where local identification fails at first order but holds at second order. Although this situation has been recognized to arise in models of interest, there are no general results available on either GMM or II estimators in this case. In this paper, we fill this gap and make the following four main contributions. First, we present the large sample distribution of the GMM estimator when the parameter vector is globally identified but only identified locally at second order. Second, we consider the large sample properties of an II estimator when local identification is only at second order. Here we focus on the II estimator defined by the following set-up: the auxiliary model consists of a set of population moment conditions indexed by a vector of auxiliary parameters and the target function for the II estimation is a GMM estimator of the auxiliary parameter vector. Within this framework, there are two types of identification conditions: one set

\footnotetext{
${ }^{5}$ Subsequent developments include the introduction of asymptotics based on either nearly-weak identification or many moments; for a recent review of this literature see Hall (2015).
} 
involving the binding function, and the other involving the auxiliary parameters. The standard firstorder asymptotic theory is premised on the assumption that the binding function satisfies global and first-order local identification conditions and the auxiliary parameters are globally and first-order locally identified within the auxiliary model. In this paper, we present the limiting distribution of the II estimator under the following two scenarios: (i) the binding function satisfies the global and first-order local identification conditions and the auxiliary parameters are globally identified but only locally identified at second order; (ii) the binding function satisfies the global identification condition but only satisfies the local identification condition at second order, and the auxiliary parameters are globally and first-order locally identified. The limit distributions of both GMM and II estimators under our conditions are shown to be non-standard but easily simulated, making it possible to perform inference about the parameters in this setting. Our third contribution is to use our results on II to analyze the large sample behaviour under second-order local identification of a version of II that has recently been shown by Frazier and Renault (2016) to be optimal within the standard first-order asymptotic framework. We find that the large sample properties of this optimal estimator are affected by the failure of the first-order local identification condition on either the binding function or the auxiliary parameters. However, interestingly, we find that use of this optimal estimator means inferences are robust to one possible source of failure of the firstorder local identification condition involving the binding function. Our fourth contribution is to examine the accuracy of our distribution theory as an approximation to finite sample behaviour in a small simulation study involving a dynamic panel data model in which the parameter of interest is identified locally at second order by a set of non-linear moment restrictions but not at first order at a particular point in the parameter space. Our simulation results indicate that the limiting distribution theory derived in our paper leads to reliable GMM/II-based inferences in moderate to large samples in the neighbourhood of this point of first-order identification failure. In contrast, inferences based on standard asymptotic theory (derived under the assumption of first-order local identification) are very misleading in this neighbourhood. Comparing GMM and II, we find our limiting distribution theory provides a reasonable approximation to the behaviour of the GMM at smaller sample sizes than it does for the II estimator, but that II exhibits smaller bias at the point of first-order local identification failure.

Our results for GMM cover all the cases cited in the paragraph before last, and our results for II cover cases in which any of the models cited in the previous paragraph are used as auxiliary model or (for those involving a distributional assumption) as simulator. ${ }^{6}$ We conjecture our results may also be relevant to estimation of certain DSGE models by GMM or II, an issue to which we return at the end of the paper.

An outline of the paper is as follows. Section 2 briefly reviews GMM and II estimation and their inference frameworks under first-order local identification. Section 3 defines second-order identification and provides two examples. Sections 4 and 5 present the limiting distribution for GMM and II estimators respectively. Section 6 reports the results from the simulation study, and Section 7 offers some concluding remarks. All proofs are relegated to an Appendix.

\footnotetext{
${ }^{6}$ Gourieroux, Phillips, and Yu (2010) suggest using II to bias correct ML. In this case, the auxiliary model is the ML estimator from the sample and is based on the same distributional assumption as the the simulator.
} 


\section{Identification and the first-order asymptotics of GMM and II}

In this section, we briefly review the basic GMM and II inference frameworks based on first-order asymptotics, paying especial attention to the role of first-order local identification. Since both methods can be viewed as special cases of "minimum chi-squared", we use the latter to unify our presentation. Therefore, we begin by defining the GMM and II estimators, and then present the minimum chi-squared framework. To this end, we introduce the following notation. In each case the model involves random vector $X$ which is assumed strictly stationary with distribution $P\left(\theta_{0}\right)$ that is indexed by a parameter vector $\theta_{0} \in \Theta \subset \mathbb{R}^{p}$. For some of the discussion only a subset of the parameters may be of primary interest, and so we write $\theta=\left(\phi^{\prime}, \psi^{\prime}\right)^{\prime}$ where $\phi \in \Phi \subset \mathbb{R}^{p_{\phi}}$ and $\psi \in \Psi \subset \mathbb{R}^{p_{\psi}}$. Throughout, $W_{T}$ denotes a positive semi-definite matrix with the dimension defined implicitly by the context.

GMM:

GMM is a partial information method in the sense that its implementation does not require knowledge of $P(\cdot)$ but only a population moment condition implied by this underlying distribution. In view of this, we suppose that $\phi_{0}$ is of primary interest and the model implies: ${ }^{7}$

$$
E\left[g\left(X, \phi_{0}\right)\right]=0,
$$

where $g(\cdot)$ is a $q \times 1$ vector of continuous functions. The GMM estimator of $\phi_{0}$ based on (1) is defined as:

$$
\hat{\phi}_{G M M}=\operatorname{argmin}_{\phi \in \Phi} Q_{T}^{G M M}(\phi),
$$

where

$$
Q_{T}^{G M M}(\phi)=T^{-1} \sum_{t=1}^{T} g\left(x_{t}, \phi\right)^{\prime} W_{T} T^{-1} \sum_{t=1}^{T} g\left(x_{t}, \phi\right),
$$

and $\left\{x_{t}\right\}_{t=1}^{T}$ represents the sample observations on $X$.

As evident from the above, GMM estimation is based on the information that the population moment $E[g(X, \phi)]$ is zero when evaluated at $\phi=\phi_{0}$. The form of this moment condition depends on the application: in economic models that fit within the framework of discrete dynamic programming models then the moment condition often takes the form of Euler equation times a vector of instruments ${ }^{8}$ in models estimated via quasi-maximum likelihood then the moment condition is the quasi-score. ${ }^{9}$

$I I$ :

II is essentially a full information method in the sense it provides a method of estimation of $\theta_{0}$ given knowledge of $P(\cdot)$. Within II, there are two models: the "simulator" which represents the model of interest - $X \sim P(\theta)$ in our notation - and an "auxiliary model" that is introduced solely as the basis for estimation of the parameters of the simulator. Although $\theta_{0}$ is unknown, data can be simulated from the simulator for any given $\theta$. To implement II, this simulation needs to be performed a

\footnotetext{
${ }^{7}$ If $p_{\psi}=0$ then $\phi=\theta$ and our presentation covers the case when the entire parameter vector is being estimated.

${ }^{8}$ For example, the consumption based asset pricing model in the seminal article by Hansen and Singleton (1982).

${ }^{9}$ For example, see Hamilton (1994) [p.428-9].
} 
number of times, $s$ say, and we denote these simulated series by $\left\{x_{t}^{(i)}(\theta)\right\}_{t=1}^{T}$ for $i=1,2, \ldots s$. The auxiliary model is estimated from the data; let $h_{T}=h\left(\left\{x_{t}\right\}_{t=1}^{T}\right)$ be some feature of this model that we refer to as the "target function", and $h_{T}^{(i)}(\theta)=h\left(\left\{x_{t}^{(i)}(\theta)\right\}_{t=1}^{T}\right)$. Assume $\operatorname{dim}\left(h_{T}\right)=\ell \geq p$. The II estimator of $\theta_{0}$ is: ${ }^{10}$

$$
\hat{\theta}_{I I}=\operatorname{argmin} Q_{T}^{I I}(\theta),
$$

where

$$
Q_{T}^{I I}(\theta)=\left[h_{T}-\frac{1}{s} \sum_{i=1}^{s} h_{T}^{(i)}(\theta)\right]^{\prime} W_{T}\left[h_{T}-\frac{1}{s} \sum_{i=1}^{s} h_{T}^{(i)}(\theta)\right] .
$$

To characterize the population analog of the information being exploited here, we assume that $h_{T} \stackrel{p}{\rightarrow} h_{0}$, for some constant $h_{0}$. Noting that there exists a mapping from $\theta_{0}$ to $h(\cdot)$ through $x_{t}\left(\theta_{0}\right)$, we can write $h_{0}=b\left(\theta_{0}\right)$ for some $b(\cdot)$, known as the binding function. Then, as Gourieroux, Monfort, and Renault (1993) observe, II exploits the information that $k\left(h_{0}, \theta_{0}\right)=h_{0}-b\left(\theta_{0}\right)=0$ in essence that, at the true parameter value, the simulator encompasses the auxiliary model.

The choice of $h(\cdot)$ varies, in practice, and depends on the setting. Examples include: raw data moments, such as the first two moments of macroeconomic or asset series, e.g. see Heaton (1995); the estimator or score vector from an auxiliary model that is in some way closely related to the simulator, ${ }^{11}$ e.g. Gallant and Tauchen (1996), Garcia, Renault, and Veredas (2011); estimated moments or parameters from the auxiliary model, such as in DSGE models, e.g. see the references in footnote 3 .

\section{Minimum chi-squared:}

As is apparent from the above definitions, both GMM and II estimation involve minimizing a quadratic form in the sample analogs to the population information about $\phi_{0}$ or $\theta_{0}$ on which they are based namely, $E\left[g\left(X, \phi_{0}\right)\right]=0$ for GMM and $k\left(h_{0}, \theta_{0}\right)=0$ for II. As such they can both be viewed as fitting within the class of minimum chi-squared. This common structure explains many of the parallels in their first-order asymptotic structure, and is also useful for highlighting the role of various identification conditions in the analyses.

Minimum chi-squared estimation is first introduced by Neyman and Pearson (1928) in the context of a specific model, but their insight is applied in more general models by Neyman (1949), Barankin and Gurland (1951) and Ferguson (1958). Suppose again that $\phi_{0}$ is of primary interest, recalling that $p_{\psi}=0$ implies $\phi=\theta$, and let $m_{T}(\phi)$ be a $n \times 1$ vector, where $n \geq p_{\phi}$, satisfying

Assumption 1. (i) $m_{T}(\phi)=O_{p}(1)$ for all $\phi \in \Phi ;$ (ii) $T^{1 / 2} m_{T}\left(\phi_{0}\right) \stackrel{d}{\rightarrow} N\left(0, V_{m}\right)$, where $V_{m}$ is a positive definite matrix of finite constants.

As a result, $T m_{T}\left(\phi_{0}\right)^{\prime} V_{m}^{-1} m_{T}\left(\phi_{0}\right) \stackrel{d}{\rightarrow} \chi_{n}^{2}$, and this structure explains the designation of the following estimator as a minimum chi-squared:

$$
\operatorname{argmin}_{\phi \in \Phi} \operatorname{Tm}_{T}(\phi)^{\prime} \hat{V}_{m}^{-1} m_{T}(\phi),
$$

\footnotetext{
${ }^{10}$ We note that II as defined in (4)-(5) is one version of the estimator. An alternative version involves simulating a single series of length $S T$. For scenarios involving optimization in the auxiliary model, this second approach has the advantage of requiring only one optimization. The first-order asymptotic properties of the II estimator are the same either way; see Gourieroux, Monfort, and Renault (1993).

${ }^{11}$ For the first-order asymptotic equivalence of these two approaches, see Gourieroux, Monfort, and Renault (1993).
} 
where $\hat{V}_{m} \stackrel{p}{\rightarrow} V_{m}$. However, for our purposes here, it is convenient to begin with the more general definition of minimum chi-squared estimator: ${ }^{12}$

$$
\hat{\phi}_{M C}=\operatorname{argmin}_{\phi \in \Phi} Q_{T}(\phi),
$$

where

$$
Q_{T}(\phi)=m_{T}(\phi)^{\prime} W_{T} m_{T}(\phi) .
$$

To consider the first-order asymptotic properties of minimum chi-squared estimators, we introduce a number of high level assumptions.

Assumption 2. (i) $W_{T} \stackrel{p}{\rightarrow} W$, a positive definite matrix of constants; (ii) $\Phi$ is a compact set; (iii) $Q_{T}(\phi) \stackrel{p}{\rightarrow} Q(\phi)=m(\phi)^{\prime} W m(\phi)$ uniformly in $\phi ;(i v) Q(\phi)$ is continuous on $\Phi ;(v) Q\left(\phi_{0}\right)<Q(\phi)$ $\forall \phi \neq \phi_{0}, \phi \in \Phi$.

Assumption 2(v) serves as a global identification condition. These conditions are sufficient to establish consistency; for example see Newey and McFadden (1994).

Proposition 1. If Assumption 2 holds then $\hat{\phi}_{M C} \stackrel{p}{\rightarrow} \phi_{0}$.

The first-order conditions of the minimization in (7) are:

$$
M_{T}\left(\hat{\phi}_{M C}\right)^{\prime} W_{T} m_{T}\left(\hat{\phi}_{M C}\right)=0,
$$

where $M_{T}(\bar{\phi})=\partial m_{T}(\phi) /\left.\partial \phi^{\prime}\right|_{\phi=\bar{\phi}}$, a matrix commonly referred to as the Jacobian in this context. These conditions are the source for the standard first-order asymptotic distribution theory of the estimator, but the latter requires the Jacobian to satisfy certain restrictions. To present these conditions, define $N_{\phi, \epsilon}$ to be an $\epsilon$-neighbourhood of $\phi_{0}$ that is, $N_{\phi, \epsilon}=\left\{\phi ;\left\|\phi-\phi_{0}\right\|<\epsilon\right\}$.

Assumption 3. (i) $\phi_{0}$ is an interior point of $\Phi$; (ii) $M_{T}(\phi) \stackrel{p}{\rightarrow} M(\phi)$ uniformly on $N_{\phi, \epsilon}$; (iii) $M(\phi)$ is continuous on $N_{\phi, \epsilon}$; (iv) $M\left(\phi_{0}\right)$ is rank $p_{\phi}$.

Assumption $3(\mathrm{iv})$ is the condition for first-order local identification. It is sufficient but not necessary for local identification of $\phi_{0}$ on $N_{\phi, \epsilon}$, but it is necessary for the development of the standard first-order asymptotic theory. Under Assumptions 1-3, the Mean Value Theorem applied to (9) yields:

$$
T^{1 / 2}\left(\hat{\phi}_{M C}-\phi_{0}\right) \simeq-\left\{M\left(\phi_{0}\right)^{\prime} W M\left(\phi_{0}\right)\right\}^{-1} M\left(\phi_{0}\right)^{\prime} W \tilde{m}_{T}\left(\phi_{0}\right),
$$

where $\simeq$ denotes equality up to terms of $o_{p}(1)$, from which the first-order asymptotic distribution follows.

Proposition 2. If Assumptions 1-3 hold then:

$$
T^{1 / 2}\left(\hat{\phi}_{M C}-\phi_{0}\right) \stackrel{d}{\rightarrow} N\left(0, V_{\phi}\right)
$$

where

$$
V_{\phi}=\left[M\left(\phi_{0}\right)^{\prime} W M\left(\phi_{0}\right)\right]^{-1} M\left(\phi_{0}\right)^{\prime} W V_{m} W M\left(\phi_{0}\right)\left[M\left(\phi_{0}\right)^{\prime} W M\left(\phi_{0}\right)\right]^{-1} .
$$

\footnotetext{
${ }^{12}$ See Ferguson (1958).
} 
As apparent, $V_{\phi}$ depends on $W$. Hansen (1982)[Theorem 3.2] shows that the choice of $W$ that minimizes $V_{\phi}$ is $W=V_{m}^{-1}$ which yields: $V_{\phi}=\left\{M\left(\phi_{0}\right)^{\prime} V_{m}^{-1} M\left(\phi_{0}\right)\right\}^{-1}$. This efficiency bound can be achieved by setting $W_{T}=\hat{V}_{m}^{-1}$ where $\hat{V}_{m} \stackrel{p}{\rightarrow} V_{m}$ to produce the version of the estimator in (6).

\section{Identification:}

Hansen (1982) provides general conditions under which the first-order asymptotic framework above goes through for GMM with

$$
m_{T}(\phi)=T^{-1} \sum_{t=1}^{T} g\left(x_{t}, \phi\right) .
$$

Gourieroux, Monfort, and Renault (1993) prove the same results for II with ${ }^{13}$

$$
m_{T}(\theta)=h_{T}-\frac{1}{s} \sum_{i=1}^{s} h_{T}^{(i)}(\theta) .
$$

We now turn to the nature and role of the identification conditions in the above analysis. ${ }^{14}$ Global identification is crucial for consistency; global and first-order local identification are needed for the asymptotic distribution theory. We now consider what these conditions imply for GMM and II estimators in turn.

For GMM, given Assumption 2(i), the global identification condition for GMM can be equivalently stated as $E[g(X, \phi)]=0$ has a unique solution at $\phi=\phi_{0}$. The first-order local identification can be stated as $E\left[\partial g(X, \phi) /\left.\partial \phi^{\prime}\right|_{\phi=\phi_{0}}\right]$ is full column rank.

For II, the situation is more subtle as the identification conditions involve the binding function and may also involve the auxiliary model depending on the nature of the target function. The binding function needs to satisfy global and first-order local identification conditions. The global identification condition is that $h_{0}-b(\theta)=0$ has a unique solution at $\theta=\theta_{0}$, and the first-order local identification condition is that $\partial b(\theta) /\left.\partial \theta^{\prime}\right|_{\theta=\theta_{0}}$ has full column rank. If the target function $h_{T}$ depends on a vector of parameters in the auxiliary model then these parameters need to be globally and first-order locally identified in order for $h_{T}$ to exhibit the large sample properties assumed above. The latter properties are: (a) $h_{T} \stackrel{p}{\rightarrow} h_{0}$; (b) $m_{T}\left(\theta_{0}\right)$ in (11) converges to a mean zero normal distribution. To show the link between these properties and the identification of the auxiliary parameter vector, we consider the case where the auxiliary model consists of a set of moment conditions indexed by a vector of auxiliary parameters, and $h_{T}$ is a sample-based GMM estimator of the vector of auxiliary parameters. In this case, condition (a) represents the GMM global identification condition of the auxiliary parameter vector in the auxiliary model. Condition (b) is established by showing that $T^{1 / 2}\left(h_{T}-h_{0}\right)$ converges to a normal distribution which in turn requires the auxiliary model to satisfy the standard GMM condition for first-order local identification of $h_{0}$ in the auxiliary model; see Gourieroux, Monfort, and Renault (1993)[p.S109]. Therefore, when $h_{T}$ depends on estimated parameters of the auxiliary model, there are two sets of identification conditions that must hold for the standard first-order asymptotic framework of II to apply:

\footnotetext{
${ }^{13}$ In spite of the similarities of the two methods, the asymptotic properties of II cannot be deduced directly from the corresponding GMM analysis because the simulation-based implementation takes II outside the GMM framework; see inter alia Duffie and Singleton (1993) or Ghysels and Guay (2003, 2004).

${ }^{14}$ The minimum chi-squared structure can also be used to explain other common features of GMM and II, see Dovonon and Hall (2015).
} 
conditions on the binding function and conditions involving the identification of the parameters in the auxiliary model. ${ }^{15}$

In linear models, global and first-order local identification are equivalent. However in nonlinear models, global identification is possible without first-order local identification because local identification can be ensured by higher order derivatives of $m(\phi)$. Under such a scenario, the parameters can be consistently estimated but the standard first-order asymptotic framework described above is not valid. For the rest of this paper, we focus on situations in which parameters are globally identified but local identification is only second-order: Section 3 defines second-order local identification and provides two examples of econometric models in which it occurs; Section 4 develops a limiting distribution theory of the GMM estimator; Section 5 characterizes the limiting behaviour of the II estimator when the target function $h_{T}$ is the GMM estimator of a set of auxiliary parameters.

\section{Second-order local identification}

For our analysis of GMM and II, we adopt the definition of second-order local identification originally introduced by Dovonon and Renault (2009). To present this definition, we introduce the following notations. Let $m(\phi)=E[g(X, \phi)]$ and

$$
M_{k}^{(2)}\left(\phi_{0}\right)=E\left[\left.\frac{\partial^{2} g_{k}(X, \phi)}{\partial \phi \partial \phi^{\prime}}\right|_{\phi=\phi_{0}}\right], \quad k=1,2 \ldots, q,
$$

where $g_{k}(X, \phi)$ is the $k^{t h}$ element of $g(X, \phi)$ and $g(\cdot)$ is defined in (1). Second-order local identification is defined as follows.

Definition 1. The moment condition $m(\phi)=0$ locally identifies $\phi_{0} \in \Phi$ up to the second order if:

(a) $m\left(\phi_{0}\right)=0$.

(b) For all $u$ in the range of $M\left(\phi_{0}\right)^{\prime}$ and all $v$ in the null space of $M\left(\phi_{0}\right)$, we have:

$$
\left(M\left(\phi_{0}\right) u+\left(v^{\prime} M_{k}^{(2)}\left(\phi_{0}\right) v\right)_{1 \leq k \leq q}=0\right) \Rightarrow(u=v=0)
$$

This condition is derived using a second order expansion of $m(\phi)$ around $m\left(\phi_{0}\right)$ and can be motivated as follows. ${ }^{16}$ For any non-zero $\phi-\phi_{0}$ with $\phi \in N_{\phi, \epsilon}$, we have $\phi-\phi_{0}=c_{1} u+c_{2} v$ where $c_{1}, c_{2}$ are constants such that $c_{1} \neq 0$ and/or $c_{2} \neq 0 .{ }^{17}$ For those directions for which $c_{1}$ is non-zero then the first order term is non-zero and dominates, and for those directions in which $c_{1}=0$, then the second-order term is non-zero. Thus, without requiring that the Jacobian matrix $M\left(\phi_{0}\right)$ to have full rank, conditions (a) and (b) in Definition 1 guarantee local identification in the sense that there is no sequence of points $\left\{\phi_{n}\right\}$ different from $\phi_{0}$ but converging to $\phi_{0}$ such that $m\left(\phi_{n}\right)=0$ for all $n$. The difference between first-order local identification and second-order local identification (with

\footnotetext{
${ }^{15}$ If $h_{T}$ involves purely raw data moments then conditions (a) and (b) hold if $T^{1 / 2}\left(h_{T}-h_{0}\right)$ satisfies the conditions for the Central Limit Theorem and so the only identification conditions are those relating to the binding function.

${ }^{16}$ We implicitly assume throughout the paper that the local dominance conditions under which expectation and derivatives can be interchanged hold whenever useful.

${ }^{17}$ Recall the range of $M\left(\phi_{0}\right)^{\prime}$ is the orthogonal complement of the null space of $M\left(\phi_{0}\right)$.
} 
$M\left(\phi_{0}\right)$ rank deficient) is how sharply $m(\phi)$ moves away from 0 in the neighborhood of $\phi_{0}$. We now consider two examples in which local identification fails at first order but holds at second order.

\section{Example 1. Nonstationary panel $A R(1)$ model with individual fixed effects.}

Consider the standard AR(1) panel data model with individual specific effects,

$$
y_{i t}=\rho y_{i, t-1}+\eta_{i}+\varepsilon_{i t}, \quad i=1, \ldots, N, \quad t=1,2 .
$$

Assume that the vector $\left(y_{i 0}, \eta_{i}, \varepsilon_{i 1}, \varepsilon_{i 2}\right)$ is i.i.d. across $i$ with mean 0 and that $E\left(\varepsilon_{i t}^{2}\right)=\sigma_{\varepsilon}^{2}$, $E\left(\varepsilon_{i s} \varepsilon_{i t}\right)=0$ for $s \neq t, s, t=1,2, E\left(\eta_{i}^{2}\right)=\sigma_{\eta}^{2}, E\left(y_{i 0}^{2}\right)=\sigma_{0}^{2}, E\left(\varepsilon_{i t} \eta_{i}\right)=0, E\left(\varepsilon_{i t} y_{i 0}\right)=0$, $t=1,2$, and $E\left(y_{i 0} \eta_{i}\right)=\sigma_{0 \eta}$. For this example, $\theta=\left(\rho, \sigma_{0}^{2}, \sigma_{\eta}^{2}, \sigma_{0 \eta}, \sigma_{\varepsilon}^{2}\right)^{\prime}$. Our primary focus here is on estimation of $\rho$ and so we partition the parameter vector as follows: $\theta=\left(\rho, \theta_{2}\right)^{\prime}$.

For $|\rho|<1$, this model can be estimated via GMM using the moment conditions in Arellano and Bond (1991). However as pointed out by Blundell and Bond (1998), the Arellano-Bond (AB) moments only provide weak identification of $\theta$ as $\rho$ tends to one. Blundell and Bond (1998) propose augmenting the $A B$ moments with an additional set of moments to produce the so-called "System GMM estimator": this approach solves the weak identification problems for $\rho$ less than one but is not valid for $\rho=1$ because the approach exploits properties of the series that only hold for $|\rho|<1$. Quasi Maximum Likelihood estimation of the model has been studied by Kruiniger (2013) for $-1<\rho<1$.

An alternative solution to the identification problems with the AB moments is to base estimation on higher moments. Expressing the variance of $y_{i}=\left(y_{i 0}, y_{i 1}, y_{i 2}\right)^{\prime}$ as a function of the model parameters, $\theta$ can be identified by the moment condition restriction:

$$
E\left[g\left(y_{i}\right)-H(\rho) \theta_{2}\right]=0,
$$

where $g(\cdot)=\left[g_{1}(\cdot)^{\prime}, g_{2}(\cdot)^{\prime}\right]^{\prime}, H(\cdot)=\left[H_{1}(\cdot)^{\prime}, H_{2}(\cdot)^{\prime}\right]^{\prime}$,

$$
\begin{gathered}
g_{1}\left(y_{i}\right)=\left(\begin{array}{l}
y_{i 0} y_{i 1} \\
y_{i 0} y_{i 2}
\end{array}\right), \quad g_{2}\left(y_{i}\right)=\left(\begin{array}{c}
y_{i 0}^{2} \\
y_{i 1}^{2} \\
y_{i 1} y_{i 2} \\
y_{i 2}^{2}
\end{array}\right), \\
H_{1}(\rho)=\left(\begin{array}{cccc}
\rho & 0 & 1 & 0 \\
\rho^{2} & 0 & 1+\rho & 0
\end{array}\right), \quad H_{2}(\rho)=\left(\begin{array}{cccc}
1 & 0 & 0 & 0 \\
\rho^{2} & 1 & 2 \rho & 1 \\
\rho^{3} & 1+\rho & \rho(1+2 \rho) & \rho \\
\rho^{4} & (1+\rho)^{2} & 2 \rho^{2}(1+\rho) & 1+\rho^{2}
\end{array}\right) .
\end{gathered}
$$

Note that $\mathrm{H}_{2}(\rho)$ is nonsingular for all $\rho \neq 0$ and we have:

$$
\theta_{2}(\rho)=H_{2}^{-1}(\rho) E\left[g_{2}\left(y_{i}\right)\right] .
$$

Using (13) involving $g_{1}(\cdot)$, we can therefore consider the moment condition:

$$
E\left[g_{1}\left(y_{i}\right)-H_{1}(\rho)\left(H_{2}(\rho)\right)^{-1} g_{2}\left(y_{i}\right)\right]=0
$$

for inference about $\rho$, our main parameter of interest. The true parameter value that we consider for the data generating process is $\theta^{*}=\left(1, \theta_{2}^{*}\right)$, with $\theta_{2}^{*}=\left(\sigma_{0}^{*^{2}}, 0,0, \sigma_{\varepsilon}^{*^{2}}\right)^{\prime}$. In the appendix, it is shown that (14) globally identifies $\rho$ but local identification fails at first order and holds at second order. 
While the above discussion has concentrated on GMM, we note that II methods have also been proposed for dynamic panel data models. Gourieroux, Phillips, and Yu (2010) propose an II estimator in which the function $h_{T}$ is the MLE under normality. They note that the II approach can be based on nonlinear moments and, following their suggestion, II can be applied using the moments in (14) as the auxiliary model. In Section 6, we report simulation results that compare GMM based on (14) with II using (14) as the auxiliary model.

\section{Example 2. A conditionally heteroscedastic factor model}

Consider the conditionally heteroskedastic factor ( $\mathrm{CHF}$ ) model of two asset returns:

$$
\left(\begin{array}{l}
y_{1 t} \\
y_{2 t}
\end{array}\right)=\left(\begin{array}{l}
\gamma_{1} \\
\gamma_{2}
\end{array}\right) f_{t}+\left(\begin{array}{l}
u_{1 t} \\
u_{2 t}
\end{array}\right)
$$

with

$$
\begin{gathered}
E\left[\left(f_{t}, u_{t}^{\prime}\right)^{\prime} \mid \mathfrak{F}_{t-1}\right]=0, \operatorname{Var}\left[f_{t} \mid \mathfrak{F}_{t-1}\right]=\sigma_{t-1}^{2}, \\
\operatorname{Var}\left[\left(u_{1 t}, u_{2 t}\right)^{\prime} \mid \mathfrak{F}_{t-1}\right]=\operatorname{Diag}\left(\Omega_{1}, \Omega_{2}\right), \operatorname{Cov}\left[f_{t}, u_{t} \mid \mathfrak{F}_{t-1}\right]=0 .
\end{gathered}
$$

In this model, $f_{t}$ is the latent common GARCH factor, $u_{t}$ is the vector of idiosyncratic shocks and $\sigma_{t-1}^{2}$ is the time varying conditional variance of $f_{t}$ where the conditioning set $\mathfrak{F}_{t}$ is an increasing filtration containing current and past values of $f_{t}$ and $y_{t}$. In addition to this specification, it is assumed that $\gamma_{1} \neq 0$ and $\gamma_{2} \neq 0$, meaning that the two asset return processes are conditionally heteroskedastic. Conditions for the identification of the factor structure ${ }^{18}(15)-(16)$ can be found in Doz and Renault (2004). The parameter vector of interest is $\theta \equiv\left(\gamma_{1}, \gamma_{2}, \Omega_{1}, \Omega_{2}\right)^{\prime}$.

This model has been introduced by Diebold and Nerlove (1989) and further studied by Fiorentini, Sentana, and Shephard (2004) and Doz and Renault (2006). Fiorentini, Sentana, and Shephard (2004) impose additional structure on the model and propose a Markov chain Monte Carlo approach to estimation. Doz and Renault (2006) propose a GMM approach based on moment conditions that identify the parameters up to one $\left(\right.$ say, $\left.\gamma_{1}\right)$ that is given a 'reasonable' value. This partial identification is the cost of allowing $\operatorname{Var}\left[\left(u_{1 t}, u_{2 t}\right)^{\prime} \mid \mathfrak{F}_{t-1}\right]$ to be non-diagonal. Here, we consider an II estimator for the model. The simulator is (15)-(16) and the assumption that $\left(f_{t}, u_{t}^{\prime}\right)^{\prime}$ is conditionally normally distributed.

The auxiliary model is defined as:

$$
\begin{aligned}
E\left[\left(\begin{array}{c}
1 \\
z_{t-1}
\end{array}\right)\left\{\left(y_{1 t}-\delta y_{2 t}\right\}^{2}-c\right]\right] & =0 \\
E\left[y_{1 t}^{2}\right] & =b_{1} \\
E\left[y_{2 t}^{2}\right] & =b_{2} \\
E\left[y_{1 t} y_{2 t}\right] & =b_{3} .
\end{aligned}
$$

where $z_{t-1} \in \mathfrak{F}_{t-1}$, (e.g lagged square returns), $\delta=\gamma_{1} / \gamma_{2}, b_{1}=\gamma_{1}^{2}+\Omega_{1}, b_{2}=\gamma_{2}^{2}+\Omega_{2}, b_{3}=$ $\gamma_{1} \gamma_{2}, c=\Omega_{1}+\delta^{2} \Omega_{2}$, and $c=b_{1}+\delta^{2} b_{2}-2 \delta b_{3}$. The parameter vector in the auxiliary model,

\footnotetext{
${ }^{18}$ The conditionally heteroskedastic factor representation (15)-(16) for $y_{t}$ is uniquely determined if we restrict to decompositions such that the factor has unit variance and non degenerate conditional variance with no positive lower bound, that is: $E\left(\sigma_{t}^{2}\right)=1, \operatorname{Var}\left(\sigma_{t}^{2}\right)>0$ and $P\left(\sigma_{t}^{2}>\sigma^{2}\right)<1$ for all $\sigma^{2}>0$. In this case, $\Omega$ is uniquely determined and $\gamma=\left(\gamma_{1}, \gamma_{2}\right)^{\prime}$ is identified up to the sign. Restricting $\gamma_{1}>0$ completes the identification of the factor structure. We refer to Doz and Renault (2004) for a more detailed discussion on the identification of CHF models.
} 
$h=\left(b_{1}, b_{2}, b_{3}, \delta, c\right)^{\prime}$, is globally identified. In addition, the parameter $\theta$ of the structural model can be determined from $h$ (enforcing $\gamma_{1}>0$ ) as follows:

$$
\theta_{1} \equiv \gamma_{1}=\sqrt{\delta b_{3}}, \quad \theta_{2} \equiv \gamma_{2}=\sqrt{\frac{b_{3}}{\delta}}, \quad \theta_{3} \equiv \Omega_{1}=b_{1}-\delta b_{3}, \quad \theta_{4} \equiv \Omega_{2}=b_{2}-\frac{b_{3}}{\delta} .
$$

However, as shown in the appendix, $h$ is not locally identified at first order but is at second order.

\section{The limiting distribution of the GMM estimator}

In this section, we consider the moment condition model (1) and study the asymptotic behaviour of the GMM estimator when $\phi_{0}$ is second-order locally identified because the moment condition exhibits the properties in Definition 1 but the standard local identification condition (Assumption 3(iv)) fails. ${ }^{19}$

We study the asymptotic behaviour of the GMM estimator by restricting ourselves to the case of one-dimension rank deficiency, i.e. the rank of $M\left(\phi_{0}\right)$ is equal to $p_{\phi}-1$, since this seems to be the only case that is analytically tractable. We start by considering the case where the rank deficiency is due to the last column of the Jacobian being null. To this end, we partition $\phi$ into $\left(\phi_{1: p_{\phi}-1}^{\prime}, \phi_{p_{\phi}}\right)^{\prime}$ where $\phi_{1: p_{\phi}-1}$ is the vector consisting of the first $p_{\phi}-1$ elements of $\phi$ and $\phi_{p_{\phi}}$ is the $p_{\phi}{ }^{t h}$ element of $\phi$. For ease of presentation below, we shorten the subscript and write $\phi_{1}$ for $\phi_{1: p_{\phi}-1}$. Thus $\phi_{0}=\left(\phi_{0,1}^{\prime}, \phi_{0, p_{\phi}}\right)^{\prime}$ where $\phi_{0,1}$ is a $\left(p_{\phi}-1\right) \times 1$ vector containing the true values of $\phi_{1: p_{\phi}-1}$ and $\phi_{0, p_{\phi}}$ is the true value of $\phi_{p_{\phi}}$. If $M\left(\phi_{0}\right)$ has rank $p_{\phi}-1$ with $\frac{\partial m}{\partial \phi_{p_{\phi}}}\left(\phi_{0}\right)=0$, second-order local identification is equivalent to:

$$
\operatorname{Rank}\left(\frac{\partial m}{\partial \phi_{1}^{\prime}}\left(\phi_{0}\right) \quad \frac{\partial^{2} m}{\partial \phi_{p_{\phi}}^{2}}\left(\phi_{0}\right)\right)=p_{\phi}
$$

This is the setting studied by Sargan (1983) for the instrumental variables estimator in nonlinear in parameters model.

We now present the regularity conditions under which we derive the asymptotic distribution of the GMM estimator. Define $D=\frac{\partial m}{\partial \phi_{1}^{\prime}}\left(\phi_{0}\right)$ and $G=\frac{\partial^{2} m}{\partial \phi_{p_{\phi}}^{2}}\left(\phi_{0}\right)$. The following condition states formally the identification pattern described above.

Assumption 4. (i) $m(\phi)=0 \Leftrightarrow \phi=\phi_{0}$; (ii) $\partial m\left(\phi_{0}\right) / \partial \phi_{p_{\phi}}=0$; (iii) $\operatorname{Rank}(D G)=p_{\phi}$.

We also require the following regularity conditions to hold.

Assumption 5. (i) $m_{T}(\phi)$ has partial derivatives up to order 3 in a neighborhood $N_{\phi, \epsilon}$ of $\phi_{0}$ and the derivatives of $m_{T}(\phi)$ converge in probability uniformly over $N_{\phi, \epsilon}$ to those of $m(\phi)$.

(ii) $\sqrt{T}\left(\begin{array}{c}m_{T}\left(\phi_{0}\right) \\ \frac{\partial m_{T}}{\partial \phi_{p_{\phi}}}\left(\phi_{0}\right)\end{array}\right) \stackrel{d}{\rightarrow}\left(\begin{array}{c}\mathbb{Z}_{0} \\ \mathbb{Z}_{1}\end{array}\right)$.

\footnotetext{
${ }^{19}$ Lee and Liao (2016) and Sentana (2015) propose an alternative approach using GMM estimation based on an augmented set of moment conditions that involve both the original moments and restrictions on their derivatives. While this approach allows the application of standard first-order asymptotic theory, the augmentation substantially increases the number of moment conditions used in the estimation which tends to lead to a deterioration in the quality of the first-order asymptotic theory as an approximation to finite sample behaviour; for example see Newey and Smith (2004).
} 
(iii) $W_{T}-W=o_{P}\left(T^{-1 / 4}\right), \frac{\partial m_{T}}{\partial \phi_{1}^{\prime}}\left(\phi_{0}\right)-D=O_{P}\left(T^{-1 / 2}\right)$, $\frac{\partial^{2} m_{T}}{\partial \phi_{p_{\phi}}^{2}}\left(\phi_{0}\right)-G=O_{P}\left(T^{-1 / 2}\right), \quad \frac{\partial^{2} m_{T}}{\partial \phi_{1}^{\prime} \partial \phi_{p_{\phi}}}\left(\phi_{0}\right)-G_{1 p_{\phi}}=o_{P}(1), \quad$ and $\quad \frac{\partial^{3} m_{T}}{\partial \phi_{p_{\phi}}^{3}}\left(\phi_{0}\right)-L=o_{P}(1)$, with $\quad G_{1 p_{\phi}}=\frac{\partial^{2} m}{\partial \phi_{1}^{\prime} \partial \phi_{p_{\phi}}}\left(\phi_{0}\right) \quad$ and $\quad L=\frac{\partial^{3} m}{\partial \phi_{p_{\phi}}^{3}}\left(\phi_{0}\right)$.

These conditions are slightly stronger than those imposed in the standard first-order asymptotic analysis. The derivation of the asymptotic distribution of the GMM estimator requires a mean value expansion of $m_{T}(\phi)$ up to the third order and the uniform convergence guaranteed by Assumption 5(i) is, in particular, useful to control the remainder of our expansions. Assumption 5(ii) gives the joint asymptotic distribution of $m_{T}\left(\phi_{0}\right)$ and $\frac{\partial m_{T}}{\partial \phi_{p_{\phi}}}\left(\phi_{0}\right)$. Under Assumption 4 and additional mild conditions on $g\left(X, \phi_{0}\right)$ and $\frac{\partial g}{\partial \phi_{p_{\phi}}}\left(X, \phi_{0}\right)$, the central limit theorem guarantees that $\left(\mathbb{Z}_{0}^{\prime}, \mathbb{Z}_{1}^{\prime}\right)^{\prime} \sim$ $N(0, v)$, with $v=\lim _{T \rightarrow \infty} \operatorname{Var}\left[\sqrt{T}\left(m_{T}\left(\phi_{0}\right)^{\prime}, \partial m_{T}\left(\phi_{0}\right)^{\prime} / \partial \phi_{p_{\phi}}\right)^{\prime}\right]$. Assumption 5 (iii) imposes the asymptotic order of magnitude of the difference between some sample dependent quantities and their probability limits. These orders of magnitude are enough to make these differences negligible in the expansions. Assumption 5(iii) is not particularly restrictive since most of the orders of magnitude imposed are guaranteed by the central limit theorem.

To facilitate the presentation of our main result in this section, we introduce the following definitions. Let $M_{d}$ be the matrix of the orthogonal projection on the orthogonal complement of $W^{1 / 2} D$ :

$$
M_{d}=I_{q}-W^{1 / 2} D\left(D^{\prime} W D\right)^{-1} D^{\prime} W^{1 / 2},
$$

where $I_{q}$ is the identity matrix of size $q$, let $P_{g}$ be the matrix of the orthogonal projection on $M_{d} W^{1 / 2} G$ :

$$
P_{g}=M_{d} W^{1 / 2} G\left(G^{\prime} W^{1 / 2} M_{d} W^{1 / 2} G\right)^{-1} G^{\prime} W^{1 / 2} M_{d},
$$

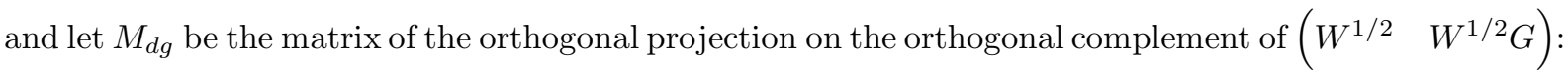

$$
M_{d g}=M_{d}-P_{g} .
$$

Let

$$
\begin{aligned}
\mathbb{R}_{1}= & \left(\mathbb{Z}_{0}^{\prime} W^{1 / 2} P_{g} W^{1 / 2} \mathbb{Z}_{0} G^{\prime}-G^{\prime} W^{1 / 2} M_{d} W^{1 / 2} \mathbb{Z}_{0} \mathbb{Z}_{0}^{\prime}\right) W^{1 / 2} M_{d} W^{1 / 2}\left(\frac{1}{3} L+G_{1 p_{\phi}} H G\right) / \sigma_{G} \\
& +\mathbb{Z}_{0}^{\prime} W^{1 / 2} M_{d g} W^{1 / 2}\left(\mathbb{Z}_{1}+G_{1 p_{\phi}} H \mathbb{Z}_{0}\right),
\end{aligned}
$$

with $\sigma_{G}=G^{\prime} W^{1 / 2} M_{d} W^{1 / 2} G$, and $H=-\left(D^{\prime} W D\right)^{-1} D^{\prime} W$.

The following result gives the asymptotic distribution of the GMM estimator $\hat{\phi}$ as defined by (3).

Theorem 1. Under Assumptions 2(i)-(iv), 4, and 5, we have:

(a) $\hat{\phi}_{1}-\phi_{0,1}=O_{P}\left(T^{-1 / 2}\right)$ and $\hat{\phi}_{p_{\phi}}-\phi_{0, p_{\phi}}=O_{P}\left(T^{-1 / 4}\right)$.

(b) If in addition, $\phi_{0}$ is interior to $\Phi$, then

$$
\sqrt{T}\left(\begin{array}{c}
\hat{\phi}_{1}-\phi_{0,1} \\
\left(\hat{\phi}_{p_{\phi}}-\phi_{0, p_{\phi}}\right)^{2}
\end{array}\right) \stackrel{d}{\rightarrow}\left(\begin{array}{c}
H \mathbb{Z}_{0}+H G \mathbb{V} / 2 \\
\mathbb{V}
\end{array}\right)
$$


with $\mathbb{V}=-2 \frac{\mathbb{Z} \mathbb{I}(\mathbb{Z}<0)}{G^{\prime} W^{1 / 2} M_{d} W^{1 / 2} G}$ and $\mathbb{Z}=G^{\prime} W^{1 / 2} M_{d} W^{1 / 2} \mathbb{Z}_{0} . \mathbb{I}(\cdot)$ is the usual indicator function.

(c) If in addition, $\mathbb{R}_{1}$ does not have an atom of probability at 0 , then:

$$
\left(\begin{array}{c}
\sqrt{T}\left(\hat{\phi}_{1}-\phi_{0,1}\right) \\
T^{1 / 4}\left(\hat{\phi}_{p_{\phi}}-\phi_{0, p_{\phi}}\right)
\end{array}\right) \stackrel{d}{\rightarrow} \mathbb{X} \equiv\left(\begin{array}{c}
H \mathbb{Z}_{0}+H G \mathbb{V} / 2 \\
(-1)^{\mathbb{B}} \sqrt{\mathbb{V}}
\end{array}\right)
$$

with $\mathbb{B}=\mathbb{I}\left(\mathbb{R}_{1} \geq 0\right)$.

The proof of this theorem is provided in Appendix. Part (a) is due to Dovonon and Renault (2009), however we provide a proof since our conditions are slightly different from theirs. Part (b) gives the asymptotic distribution of $\left[\left(\hat{\phi}_{1}-\phi_{0,1}\right)^{\prime},\left(\hat{\phi}_{p_{\phi}}-\phi_{0, p_{\phi}}\right)^{2}\right]$. This result is obtained by eliciting the $O_{P}\left(T^{-1}\right)$ terms of $m_{T}^{\prime}(\hat{\phi}) W_{T} m_{T}(\hat{\phi})$ which are collected into $K_{T}(\phi)$ as given by $(58)$ in the Appendix. The fact that $K_{T}\left(\phi_{p_{\phi}}\right)$ is a quadratic function of $\left(\phi_{p_{\phi}}-\phi_{0, p_{\phi}}\right)^{2}$ gives an intuition of the fact that only the asymptotic distribution of $\left(\hat{\phi}_{p_{\phi}}-\phi_{0, p_{\phi}}\right)^{2}$ can be obtained from this leading term of the expansion of the GMM objective function. The distribution of $\left(\hat{\phi}_{p_{\phi}}-\phi_{0, p_{\phi}}\right)$ can be obtained from Part (b) up to the sign which cannot be deduced from this leading term but rather is obtainable from the higher order, $O_{P}\left(T^{-5 / 4}\right)$, term of the objective function's expansion. We actually obtain:

$$
m_{T}^{\prime}(\hat{\phi}) W_{T} m_{T}(\hat{\phi})=K_{T}\left(\hat{\phi}_{p_{\phi}}\right)+\left(\hat{\phi}_{p_{\phi}}-\phi_{0, p_{\phi}}\right) R_{1 T}+o_{P}\left(T^{-5 / 4}\right),
$$

showing that the minimum is reached when $\left(\hat{\phi}_{p_{\phi}}-\phi_{0, p_{\phi}}\right)$ has the opposite sign to $R_{1 T}$. See (59) in Appendix for the expression of $R_{1 T}$. So long as $T R_{1 T}$, with limit distribution $\mathbb{R}_{1}$, does not vanish asymptotically, the sign of $\left(\hat{\phi}_{p_{\phi}}-\phi_{0, p_{\phi}}\right)$ can be identified by this higher order term in the expression leading to Part (c) of the theorem.

Remark 1. The continuity condition for $\mathbb{R}_{1}$ at 0 is not expected to be restrictive in general since $\mathbb{R}_{1}$ is a quadratic function of the Gaussian vector $\left(\mathbb{Z}_{0}^{\prime}, \mathbb{Z}_{1}^{\prime}\right)^{\prime}$. However, when $q=p=p_{\phi}=1$ (one moment restriction with one non first-order locally identified parameter), we can see that $\mathbb{R}_{1}=0$. In this case, the characterization of the asymptotic distribution of $T^{1 / 4}\left(\hat{\phi}-\phi_{0}\right)$ may be problematic if the estimating function is quadratic in $\phi$. Actually, $T^{1 / 4}\left(\hat{\phi}-\phi_{0}\right)$ may not have a proper asymptotic distribution in this case whereas $\sqrt{T}\left(\hat{\phi}-\phi_{0}\right)^{2}$ does have one as given by Theorem $1(b)$.

Remark 2. The asymptotic distributions in Parts (b) and (c) of Theorem 1 are both non-standard but easy to simulate. The source of randomness is $\left(\mathbb{Z}_{0}^{\prime}, \mathbb{Z}_{1}^{\prime}\right)^{\prime}$ which is typically a Gaussian vector with zero mean and asymptotic variance $v=\lim _{T \rightarrow \infty} T \operatorname{Var}\left(\begin{array}{c}m_{T}\left(\phi_{0}\right) \\ \frac{\partial m_{T}}{\partial \phi_{p_{\phi}}}\left(\phi_{0}\right)\end{array}\right)$ which can be consistently estimated by the sample variance if there are no serial correlation or by heteroskedasticity and autocorrelation consistent procedures if there are serial correlations (see Andrews, 1991). Reasonable approximations to these limiting distributions can be obtained by drawing randomly copies of $\left(\mathbb{Z}_{0}^{\prime}, \mathbb{Z}_{1}^{\prime}\right)^{\prime}$ from $N(0, \hat{v})$, where $\hat{v}$ is a consistent estimate of $v$, and using consistent estimators of $D, W, G, L$ and $G_{1 p_{\phi}}$.

Remark 3. When the moment condition model has a single parameter that is not locally identified at the first order but is at the second order, asymptotically correct $(1-\alpha)$-confidence interval, 
$1-\alpha>1 / 2$, for $\phi_{0}$ can be obtained without simulation using the following formula deduced from Theorem 1(b). The proof of asymptotic correctness is provided in Appendix.

$$
C I_{1-\alpha}\left(\phi_{0}\right)=\hat{\phi} \pm \frac{1}{T^{1 / 4}} \cdot\left(\frac{2 \sqrt{\hat{G}^{\prime} W_{T} \hat{V}_{m} W_{T} \hat{G}}}{\hat{G}^{\prime} W_{T} \hat{G}} z_{\alpha}\right)^{\frac{1}{2}}
$$

where $\hat{\phi}$ is the GMM estimator of $\phi_{0}, \hat{G}$ is a consistent estimator of $G, \hat{V}_{m}$ a consistent estimator of $V_{m}$, the long run variance of the sample moment evaluated at $\phi=\phi_{0}$, and $z_{\alpha}$ is the $(1-\alpha)$-quantile of the standard normal distribution.

Assumption 4 requires that the rank deficiency occurs in a particular way, namely because one column of the Jacobian matrix of the moment function vanishes and the other columns are linearly independent. This is only a particular form of first-order identification failure; for example it does not fit our second example in Section 3. However, as mentioned by Sargan (1983), up to a rotation of the parameter space, all rank deficient problems can be brought into this configuration as we demonstrate below.

Let $M_{0}=\frac{\partial m}{\partial \phi^{\prime}}\left(\phi_{0}\right)$ and assume that the moment condition model (1) is such that $\operatorname{Rank}\left(M_{0}\right)=$ $p_{\phi}-1$ without having a column that is equal to 0 . Let $R$ be any nonsingular $\left(p_{\phi}, p_{\phi}\right)$-matrix such that $M_{0} R_{\bullet} p_{\phi}=0$, where $R_{\bullet} p_{\phi}$ represents the last column of $R$. We can write (1) in terms of the parameter vector $\eta: \phi=R \eta$ and consider the model:

$$
m\left(R \eta_{0}\right)=E\left[g\left(X, R \eta_{0}\right)\right]=0 .
$$

By the chain rule, it follows that the population moment condition in (20) identifies $\eta_{0}=R^{-1} \phi_{0}$ with local identification properties matching Assumption 4. More precisely, setting $\eta=\left(\eta_{1}^{\prime}, \eta_{p_{\phi}}\right)^{\prime}$ where $\eta_{1}$ is $\left(p_{\phi}-1\right) \times 1$, we have:

$$
\left.\frac{\partial m(R \eta)}{\partial \eta_{p_{\phi}}}\right|_{\eta_{0}}=M_{0} R_{\bullet p_{\phi}}=0 \text { and } \operatorname{Rank}\left(\left.\frac{\partial m(R \eta)}{\partial \eta_{1}^{\prime}}\right|_{\eta_{0}}\right)=\operatorname{Rank}\left(M_{0} R_{1}\right)=p_{\phi}-1,
$$

where $R_{1}$ is the sub-matrix of the first $p_{\phi}-1$ columns of $R$. We can therefore claim that the asymptotic distribution, $\tilde{\mathbb{X}}$, of $\left(\begin{array}{c}\sqrt{T}\left(\hat{\eta}_{1}-\eta_{0,1}\right) \\ T^{1 / 4}\left(\hat{\eta}_{p_{\phi}}-\eta_{0, p_{\phi}}\right)\end{array}\right)$ is obtained by Theorem 1 with $D, G, L$, and $G_{1 p_{\phi}}$ replaced respectively by:

$$
\begin{gathered}
\tilde{D}=M_{0} R_{1}, \quad \tilde{G}=\left(R_{\bullet p_{\phi}}^{\prime} \frac{\partial^{2} m_{k}}{\partial \phi \partial \phi^{\prime}}\left(\phi_{0}\right) R_{\bullet} p_{\phi}\right)_{1 \leq k \leq q}, \quad \tilde{L}=\left(R_{\bullet p_{\phi}}^{\prime} A_{k} R_{\bullet p_{\phi}}\right)_{1 \leq k \leq q}, \\
A_{k}=\left(\frac{\partial^{3} m_{k}}{\partial \phi_{i} \partial \phi_{j} \partial \phi^{\prime}}\left(\phi_{0}\right) R_{\bullet} p_{\phi}\right)_{1 \leq i, j \leq p_{\phi}},
\end{gathered}
$$

and $\tilde{G}_{1 p_{\phi}}$, the $\left(q, p_{\phi}-1\right)$-matrix with its $k^{\text {th }}$ row equal to $R_{\bullet}^{\prime} p_{\phi} \frac{\partial^{2} m_{k}}{\partial \phi \partial \phi^{\prime}}\left(\phi_{0}\right) R_{1}$.

We use the fact that $\hat{\phi}-\phi_{0}=R\left(\hat{\eta}-\eta_{0}\right)$ to obtain the asymptotic distribution of $\hat{\phi}-\phi_{0}$. Specifically, letting $B_{T}=\left(\begin{array}{cc}\sqrt{T} I_{p_{\phi}-1} & 0 \\ 0 & T^{1 / 4}\end{array}\right)$, we obtain the asymptotic distribution of $B_{T} R^{-1}\left(\hat{\phi}-\phi_{0}\right)$ 
as that of $B_{T}\left(\hat{\eta}-\eta_{0}\right)$.

Feasible inference is possible by replacing $R$ by a consistent estimate $\hat{R}$. However, because all the components of $R^{-1}\left(\hat{\phi}-\phi_{0}\right)$ are not converging at the same rate, one needs to exercise some caution in claiming the asymptotic equivalence between $B_{T} \hat{R}^{-1}\left(\hat{\phi}-\phi_{0}\right)$ and $B_{T} R^{-1}\left(\hat{\phi}-\phi_{0}\right)$. Clearly,

$$
B_{T} \hat{R}^{-1}\left(\hat{\phi}-\phi_{0}\right)=B_{T} R^{-1}\left(\hat{\phi}-\phi_{0}\right)+\epsilon_{T}
$$

$\epsilon_{T}=-B_{T} \hat{R}^{-1}(\hat{R}-R) R^{-1}\left(\hat{\phi}-\phi_{0}\right)$. But $\epsilon_{T}$ does not always vanish asymptotically. We distinguish two cases:

Case 1: $\hat{R}-R=o_{P}\left(T^{-1 / 4}\right)$. This is the case, for example, if $R$ does not depend on $\phi_{0}$ and $\hat{R}$ is a smooth function of sample means of the data. In such a case we typically have $\hat{R}-R=O_{P}\left(T^{-1 / 2}\right)$. By the Cauchy-Schwarz inequality, we have:

$$
\left\|\epsilon_{T}\right\| \leq\left\|\hat{R}^{-1}\right\|\left\|T^{1 / 4}(\hat{R}-R)\right\|\left\|T^{1 / 4} R^{-1}\left(\hat{\phi}-\phi_{0}\right)\right\|=O_{P}(1) o_{P}(1) O_{P}(1),
$$

and this remainder is negligible so that:

$$
B_{T} \hat{R}^{-1}\left(\hat{\phi}-\phi_{0}\right) \stackrel{d}{\rightarrow} \tilde{\mathbb{X}}
$$

Case 2: $\hat{R}-R=O_{P}\left(T^{-1 / 4}\right)$. This is expected, for example, if $R$ is a function of $\phi_{0}$, i.e. $R \equiv R\left(\phi_{0}\right)$. If $R(\cdot)$ is continuously differentiable in a neighborhood of $\phi_{0}$, we can show (see Appendix) that:

$$
\epsilon_{T}=-A \sqrt{T}\left(\hat{\eta}_{p_{\phi}}-\eta_{0, p_{\phi}}\right)^{2}+o_{P}(1)
$$

with

$$
A=\left(\begin{array}{cc}
I_{p_{\phi}-1} & 0 \\
0 & 0
\end{array}\right) R^{-1} \frac{\partial R_{\bullet p_{\phi}}}{\partial \phi^{\prime}}\left(\phi_{0}\right) R_{\bullet} p_{\phi}
$$

Hence, we have:

$$
B_{T} \hat{R}^{-1}\left(\hat{\phi}-\phi_{0}\right) \stackrel{d}{\rightarrow} \tilde{\mathbb{X}}-A\left(\tilde{\mathbb{X}}_{p_{\phi}}\right)^{2}
$$

To perform inference about $\phi_{0}$ in the current local identification set up, it is essential to estimate a relevant reparameterization matrix $R$ that may depend on the model parameter $\phi$. In many cases, including Examples 1 and 2, it is possible, by examining the population Jacobian matrix at the true value, to figure out that it is of rank $p_{\phi}-1$ at $\phi_{0}$ because a non-zero vector $R_{\bullet} p_{\phi}\left(\phi_{0}\right)$ is found in its null space. At most, $R_{\bullet} p_{\phi}\left(\phi_{0}\right)$ is a moment function depending on $\phi_{0}$ that can be consistently estimated by sample moments evaluated at $\hat{\phi}$ providing, therefore, a consistent estimator $\hat{R}_{\bullet} p_{\phi}(\hat{\phi})$. This vector can be completed by the first $p_{\phi}-1$ elements of the canonical basis of $\mathbb{R}^{p_{\phi}}$ that do not linearly determine $\hat{R}_{\bullet} p_{\phi}(\hat{\phi})$ to obtain an estimate $\hat{R}(\hat{\phi})$ of the full reparameterization matrix $R\left(\phi_{0}\right)$. Examples 1 and 2 are further studied below to determine their respective reparameterization matrices.

In other cases, one may rely on testing to determine whether the rank of the Jacobian matrix at $\phi_{0}$ is $p_{\phi}-1$. The rank test of Wright (2003) can serve this purpose. In this case, the estimation of an element of the null space of the Jacobian is a bit more involved. Assuming that the first $p_{\phi}-1$ columns of $E\left(\frac{\partial g\left(X, \phi_{0}\right)}{\partial \phi^{\prime}}\right)$ are linearly independent. Then, in a similar spirit as the augmented 
regression of Arellano, Hansen, and Sentana (2012), we can find an element $R_{\bullet} p_{\phi}$ in its null space such that $R_{\bullet p_{\phi}}=\left(r^{\prime},-1\right)^{\prime} \in \mathbb{R}^{p_{\phi}-1} \times \mathbb{R}$, with $r$ defined by:

$$
E\left[\frac{\partial g\left(X, \phi_{0}\right)}{\partial \phi_{1}^{\prime}} r-\frac{\partial g\left(X, \phi_{0}\right)}{\partial \phi_{p_{\phi}}}\right]=0 .
$$

Letting $\Gamma_{1}(\phi)=E\left[\frac{\partial g(X, \phi)}{\partial \phi_{1}^{\prime}}\right]$ and $\Gamma_{p_{\phi}}(\phi)=E\left[\frac{\partial g(X, \phi)}{\partial \phi_{p_{\phi}}}\right]$, we have: $r=\mathbf{r}\left(\phi_{0}\right)$ with

$$
\mathbf{r}(\phi)=\left(\Gamma_{1}(\phi)^{\prime} \Gamma_{1}(\phi)\right)^{-1} \Gamma_{1}(\phi)^{\prime} \Gamma_{p_{\phi}}(\phi) .
$$

Hence, $r$ can be estimated by plug-in where $\phi_{0}$ is replaced by $\hat{\phi}$ and the population means are replaced by sample means:

$$
\left.\hat{r}=\hat{\mathbf{r}}(\hat{\phi})=\left(\hat{\Gamma}_{1}(\hat{\phi})^{\prime} \hat{\Gamma}_{1}(\hat{\phi})\right)^{-1} \hat{\Gamma}_{1}(\hat{\phi})^{\prime} \hat{\Gamma}_{p_{\phi}}\right)(\hat{\phi})
$$

where $\hat{\Gamma}_{1}(\hat{\phi})=T^{-1} \sum_{t=1}^{T} \partial g\left(x_{t}, \phi\right) /\left.\partial \phi_{1}^{\prime}\right|_{\phi=\hat{\phi}}$ and $\hat{\Gamma}_{p_{\phi}}(\hat{\phi})=T^{-1} \sum_{t=1}^{T} \partial g\left(x_{t}, \phi\right) /\left.\partial \phi_{p_{\phi}}\right|_{\phi=\hat{\phi}}$. This leads to an estimate $\hat{R}_{\bullet} p_{\phi}(\hat{\phi})$ that can be completed as described above to obtain an estimate $\hat{R}$ of $R$. If $\hat{R}-R=o_{P}\left(T^{-1 / 4}\right)$, then valid inference can be carried out about $\phi_{0}$ using (22). This is the case for instance when $\Gamma_{1}\left(\phi_{0}\right)$ and $\Gamma_{p_{\phi}}\left(\phi_{0}\right)$ do not depend on $\phi_{0}$. However, one would in general expect that $\hat{R}-R=O_{P}\left(T^{-1 / 4}\right)$ and inference will be based on (24). In this case, a consistent estimate of $A$ can be obtained as follows. Note that $\frac{\partial R_{\boldsymbol{p}_{\phi}}}{\partial \phi^{\prime}}\left(\phi_{0}\right)=\left(\frac{\partial \mathbf{r}^{\prime}}{\partial \phi}\left(\phi_{0}\right) 0\right)^{\prime}$. Letting $\phi_{k}$ be the $k^{\text {th }}$ component of $\phi,{ }^{20} \quad \Gamma_{j} \equiv \Gamma_{j}(\phi)$ and $\Gamma_{j, k} \equiv \frac{\partial \Gamma_{j}}{\partial \phi_{k}}(\phi)$, for $j=1, p_{\phi}$ and $k=1, \ldots, p_{\phi}$, we have:

$$
\frac{\partial \mathbf{r}}{\partial \phi_{k}}(\phi)=\left(\Gamma_{1}^{\prime} \Gamma_{1}\right)^{-1}\left\{-\left(\Gamma_{1, k}^{\prime} \Gamma_{1}+\Gamma_{1}^{\prime} \Gamma_{1, k}\right)\left(\Gamma_{1}^{\prime} \Gamma_{1}\right)^{-1} \Gamma_{1} \Gamma_{p_{\phi}}+\Gamma_{1, k}^{\prime} \Gamma_{p_{\phi}}+\Gamma_{1}^{\prime} \Gamma_{p_{\phi}, k}\right\} .
$$

The derivative $\partial \mathbf{r} / \partial \phi_{k}\left(\phi_{0}\right)$ can be estimated consistently via a similar plug-in approach as for $\mathbf{r}\left(\phi_{0}\right)$ above.

Returning to our examples we can see that in Example 1 of Section 3, the Jacobian matrix of the moment function (14) is null, hence, Theorem 1 applies to the GMM estimator of this model without a need for reparameterization. In Example 2 of the same section, the Jacobian matrix of the auxiliary model at the true parameter value is:

$$
\left(\begin{array}{ccccc}
0 & 0 & 0 & -2\left(b_{3}-\delta b_{2}\right) & -1 \\
0 & 0 & 0 & -2\left(b_{3}-\delta b_{2}\right) E\left(z_{t-1}\right) & -E\left(z_{t-1}\right) \\
-1 & 0 & 0 & 0 & 0 \\
0 & -1 & 0 & 0 & 0 \\
0 & 0 & -1 & 0 & 0
\end{array}\right)
$$

Recall that $b_{3}-\delta b_{2}=-\Omega_{2} \neq 0$. The null space of this matrix contains $R_{5}=\left(0,0,0,-1,2\left(b_{3}-\delta b_{2}\right)\right)^{\prime}$ and, by completing this vector by the elements of the canonical basis of $\mathbb{R}^{5}$, we obtain a relevant

\footnotetext{
${ }^{20}$ This involves a slight abuse of notation: in the formula for $\Gamma_{j, k} \partial \mathbf{r} / \partial \phi_{k}, \phi_{1}$ denotes the first element of $\phi$ and not $\phi_{1: p_{\phi}-1}$ as above.
} 
reparameterization matrix along with a consistent estimator as:

$$
R=\left(\begin{array}{ccccc}
1 & 0 & 0 & 0 & 0 \\
0 & 1 & 0 & 0 & 0 \\
0 & 0 & 1 & 0 & 0 \\
0 & 0 & 0 & 1 & -1 \\
0 & 0 & 0 & 0 & 2\left(b_{3}-\delta b_{2}\right)
\end{array}\right) \quad \text { and } \quad \hat{R}=\left(\begin{array}{ccccc}
1 & 0 & 0 & 0 & 0 \\
0 & 1 & 0 & 0 & 0 \\
0 & 0 & 1 & 0 & 0 \\
0 & 0 & 0 & 1 & -1 \\
0 & 0 & 0 & 0 & 2\left(\hat{b}_{3}-\hat{\delta} \hat{b}_{2}\right)
\end{array}\right)
$$

respectively. The asymptotic distribution for the auxiliary estimator is given by (24) which can be used to build inference about the indirect inference estimator along with Theorem 2 in the next section.

We now describe how the above results can be used to perform inference about the parameters. Our discussion focuses on Case 1 above but the methods can also be applied in settings covered by Case 2. Suppose first it is desired to construct a confidence set for $\phi_{0}$. Under the mild assumption that $\|\tilde{\mathbb{X}}\|^{2}$ has a continuous distribution at $c_{1-\alpha}$, it follows from (22) via the Continuous Mapping Theorem that

$$
\lim _{T \rightarrow \infty} P\left(\left\|B_{T} \hat{R}^{-1}\left(\hat{\phi}-\phi_{0}\right)\right\|^{2} \leq c_{1-\alpha}\right)=1-\alpha,
$$

where $c_{1-\alpha}$ is the $100(1-\alpha)^{t h}$ percentile of $\|\tilde{\mathbb{X}}\|^{2}$. Thus,

$$
\left\{\phi,\left\|B_{T} \hat{R}^{-1}(\hat{\phi}-\phi)\right\|^{2} \leq c_{1-\alpha}\right\}
$$

represents a $100(1-\alpha) \%$ confidence set for $\phi_{0}$. To derive a confidence interval for the $j^{\text {th }}$ element of $\phi_{0}, \phi_{0, j},{ }^{21}$ we re-write (22) as

$$
\hat{\phi}-\phi_{0} \stackrel{a}{\sim} \hat{R} B_{T}^{-1} \tilde{\mathbb{X}}
$$

where $\stackrel{a}{\sim}$ denotes "asymptotically distributed as". A confidence interval for $\phi_{0, j}$ can be built using simulation of $\tilde{\mathbb{X}}$ as follows: simulate $\tilde{\mathbb{X}}$ to obtain the simulated distribution of $\left[\hat{R} B_{T}^{-1} \tilde{\mathbb{X}}\right]_{j}$, the $j^{\text {th }}$ element of $\hat{R} B_{T}^{-1} \tilde{\mathbb{X}}$. To construct an equal-tailed confidence interval, calculate the $c_{j}(\alpha / 2)$ and $c_{j}(1-\alpha / 2)$, respectively the $100(\alpha / 2)^{t h}$ and $100(1-\alpha / 2)^{t h}$ percentiles of the simulated distribution of $\left[\hat{R} B_{T}^{-1} \tilde{\mathbb{X}}\right]_{j}$. A $100(1-\alpha) \%$ confidence interval is:

$$
\left\{\phi_{j}, c_{j}(\alpha / 2)<\phi_{j}<c_{j}(1-\alpha / 2)\right\} .
$$

The results in Theorem 1 can also be used to test hypotheses of the form $H_{0}: f\left(\phi_{0}\right)=0$ versus $H_{1}: f\left(\phi_{0}\right) \neq 0$ where $f(\cdot)$ is a $K \times 1$ vector of two times continuously differentiable functions. In the appendix, it is shown that:

$$
f(\hat{\phi})-f\left(\phi_{0}\right) \stackrel{a}{\sim} \frac{\partial f}{\partial \phi^{\prime}}(\hat{\phi}) \hat{R} B_{T}^{-1} \tilde{\mathbb{X}}-\frac{1}{2} \frac{1}{\sqrt{T}}\left(\hat{R}_{\bullet p_{\phi}}^{\prime} \frac{\partial^{2} f_{k}}{\partial \phi \partial \phi^{\prime}}(\hat{\phi}) \hat{R}_{\bullet p_{\phi}} \tilde{\mathbb{X}}_{p_{\phi}}^{2}\right)_{1 \leq k \leq K} \equiv V_{T}(\hat{\phi}, \tilde{\mathbb{X}}) .
$$

Quantiles of $V_{T}(\hat{\phi}, \tilde{\mathbb{X}})$ can be simulated from copies of $\tilde{\mathbb{X}}$. Note that if $f$ is a linear function then the second order term in (27) drops out and

$$
V_{T}(\hat{\phi}, \tilde{\mathbb{X}})=\frac{\partial f}{\partial \phi^{\prime}}(\hat{\phi}) \hat{R} B_{T}^{-1} \tilde{\mathbb{X}}
$$

\footnotetext{
${ }^{21}$ This involves a slight abuse of notation as here $\phi_{0,1}$ denotes the first element of $\phi_{0,1}$ and not the $\left(p_{\phi}-1 \times 1\right)$ sub-vector of $\phi_{0}$ as above.
} 


\section{The limiting distribution of the II estimator}

In this section, we turn attention to the II estimator defined by (4) and (5) in the case where the auxiliary model consists of a set of moment conditions indexed by the auxiliary parameter vector $h_{0}$ and the target function, $h_{T}$, is a GMM estimator of $h_{0}$. It can be recalled from Section 2 that within this scenario, the standard first order asymptotic theory for II estimators is premised on identification conditions involving both the binding function and the auxiliary model. We consider two scenarios. First, we derive the asymptotic distribution of the II estimator in the case where the auxiliary parameter vector is only identified locally at second order but the other conditions highlighted in Section 2 still hold. Second, we derive the asymptotic distribution of the II estimator in the case where the local identification condition associated with the binding function fails at first order but holds at second order, and the other conditions highlighted in Section 2 still hold. The section concludes by using our results to explore the large sample properties of the optimal version of II recently proposed by Frazier and Renault (2016).

\subsection{Auxiliary model is identified locally at second order:}

Suppose the auxiliary model consists of the following set of $q$ population moment conditions

$$
m^{(a)}\left(h_{0}\right)=E\left[g\left(X, h_{0}\right)\right]=0,
$$

where $g(\cdot)$ a $q \times 1$ vector of continuous functions. We use the superscript " $(a)$ " here, and below, to indicate quantities pertaining to the auxiliary model. Let $\mathcal{H} \subset \mathbb{R}^{\ell}$ denote the auxiliary parameter space. The target function $h_{T}$ is the GMM estimator of $h_{0}$ based on (28) that is,

$$
h_{T}=\operatorname{argmin}_{h \in \mathcal{H}} Q_{T}^{(a)}(h)
$$

where

$$
Q_{T}^{(a)}(h)=m_{T}^{(a)}(h)^{\prime} W_{T} m_{T}^{(a)}(h),
$$

$m_{T}^{(a)}(h)=T^{-1} \sum_{t=1}^{T} g\left(x_{t}, h\right)$ and $W_{T}$ is the weighting matrix.

Adapting the notation from Section 2 , let $h_{T}^{(i)}(\theta)$ denote the analogous GMM estimator to $h_{T}$ only based on $\left\{x_{t}^{(i)}(\theta)\right\}_{t=1}^{T}$ instead of $\left\{x_{t}\right\}_{t=1}^{T}$. The II estimator of $\theta_{0}$ is

$$
\hat{\theta}_{I I}=\operatorname{argmin}_{\theta \in \Theta} Q_{T}^{I I}(\theta),
$$

where

$$
Q_{T}^{I I}(\theta)=m_{I T}(\theta)^{\prime} \Omega_{T} m_{I T}(\theta),
$$

$m_{I T}(\theta)=h_{T}-\frac{1}{s} \sum_{i=1}^{s} h_{T}^{(i)}(\theta)$ and we have used $\Omega_{T}$ to denote the weighting matrix to distinguish it from for the weighting matrix used in the estimation of $h_{T}$.

To present the necessary regularity conditions, we introduce the following notation. We partition the auxiliary parameter vector as $h=\left(h_{1: \ell-1}^{\prime}, h_{\ell}\right)^{\prime}$ where $h_{\ell}$ is the $\ell^{t h}$ element of $h$ but for ease of notation in the formulae below set $h_{1}=h_{1: \ell-1}$. Define $M^{(a)}\left(h_{0}\right)=E\left[\partial g(X, h) /\left.\partial h^{\prime}\right|_{h=h_{0}}\right]$ and $G^{(a)}=E\left[\partial^{2} g(X, h) /\left.\partial h_{\ell}^{2}\right|_{h=h_{0}}\right]$.

The auxiliary model is assumed to satisfy the following conditions. 
Assumption 6. (i) $m^{(a)}(h)=0 \Leftrightarrow h=h_{0}$, where $h_{0}$ is an interior point of the compact set $\mathcal{H}$; (ii) $Q_{T}^{(a)}(h)$ converges uniformly to $Q^{(a)}(h)=m^{(a)}(h)^{\prime} W m^{(a)}(h)$ on $\mathcal{H}$ where $W$ is positive definite; (iii) $M^{(a)}\left(h_{0}\right)=\left[D^{(a)}, 0_{q \times 1}\right]$ where $D^{(a)}$ is a $q \times(\ell-1)$ matrix of constants with full column rank and $0_{q \times 1}$ is the $q \times 1$ null vector; (iv) $G^{(a)}$ is a vector of finite constants and rank $\left\{\left[D^{(a)}, G^{(a)}\right]\right\}=\ell ;(v)$ $m_{T}^{(a)}(h)$ has partial derivatives up to order 3 in an $\epsilon$-neighbourhood of $h_{0}, N_{h, \epsilon}$, and the derivatives of $m_{T}^{(a)}(h)$ converge uniformly over $N_{h, \epsilon}$ to those of $m^{(a)}(h)$;

(vi) $\sqrt{T}\left(\begin{array}{c}m_{T}^{(a)}\left(h_{0}\right) \\ \frac{\partial m_{T}^{(a)}}{\partial \phi_{h_{\ell}}}\left(h_{0}\right)\end{array}\right) \stackrel{d}{\rightarrow}\left(\begin{array}{c}\mathbb{Z}_{0} \\ \mathbb{Z}_{1}\end{array}\right)$;

(vii) $W_{T}-W=o_{P}\left(T^{-1 / 4}\right), \frac{\partial m_{T}^{(a)}}{\partial h_{1}^{\prime}}\left(h_{0}\right)-D^{(a)}=O_{P}\left(T^{-1 / 2}\right), \quad \frac{\partial^{2} m_{T}^{(a)}}{\partial h_{\ell}^{2}}\left(h_{0}\right)-G^{(a)}=O_{P}\left(T^{-1 / 2}\right)$, $\frac{\partial^{2} m_{T}^{(a)}}{\partial h_{1}^{\prime} \partial h_{\ell}}\left(h_{0}\right)-G_{1, \ell}^{(a)}=o_{P}(1), \frac{\partial^{3} m_{T}^{(a)}}{\partial h_{\ell}^{3}}\left(\phi_{0}\right)-L^{(a)}=o_{P}(1)$, where $W, G_{1, \ell}^{(a)}=\frac{\partial^{2} m^{(a)}}{\partial h_{1}^{\prime} \partial h_{\ell}}\left(h_{0}\right)$, and $L^{(a)}=$ $\frac{\partial^{3} m^{(a)}}{\partial h_{\ell}^{3}}\left(h_{0}\right)$ are all matrices of finite constants.

It can be recognized that under Assumption 6 the auxiliary model identifies $h_{0}$ globally but only at second order locally, and also satisfies the regularity conditions for Theorem 1 . Thus, in Theorem 2 below, we present the limiting distribution theory for II estimator in the case where the failure in first-order local identification of the auxiliary parameter is due to the last column of the Jacobian in the auxiliary model being null. We discuss the extension of this result to other patterns of identification failure in Remark 4 below.

Let $\mathbb{R}_{1}^{(a)}$ be the random variable obtained by replacing $G, D, G_{1 p_{\phi}}$ and $L$ on the right-hand side of (18) by $G^{(a)}, D^{(a)}, G_{1, \ell}^{(a)}$ and $L^{(a)}$ respectively.

Assumption 7. $\mathbb{R}_{1}^{(a)}$ has no atom of probability at 0 .

Under Assumptions 6 and 7, the limiting distribution of the GMM estimator of the auxiliary parameter vector is characterized by Theorem $1(\mathrm{c})$.

We now define the binding function associated with our framework. To this end, recall that the simulator takes the form $X \sim P(\theta)$ where $P(\cdot)$ is given and $\theta \in \Theta$. Let $E_{\theta}[\cdot]$ denote the expectations operator relative to $P(\theta)$. Defining,

$$
Q^{(a)}(h ; \theta)=E_{\theta}[g(X, h)]^{\prime} W E_{\theta}[g(X, h)],
$$

the binding function, denoted $b(\theta)$, is given by:

$$
b(\theta)=\operatorname{argmin}_{h \in \mathcal{H}} Q^{(a)}(h ; \theta) .
$$

Define $M_{I T}(\theta)=\partial m_{I T}(\theta) / \partial \theta^{\prime}$. Regularity conditions on the simulator, binding function and II minimand are given in the following assumption.

Assumption 8. (i) $X \sim P\left(\theta_{0}\right)$ where $\theta_{0}$ is an interior point of the compact set $\Theta \subset \mathbb{R}^{p}$, and the simulated series $\left\{x_{t}^{(i)}(\theta), t=1,2, \ldots, T\right\}_{i=1}^{s}$ are generated by drawing from $P(\theta) ;(i i) b: \theta \rightarrow b(\theta)$ is an injective mapping; (iii) $b(\theta)$ is continuous on $\Theta$ and differentiable on $N_{\theta, \epsilon}$, an $\epsilon$-neighbourhood of $\theta_{0} ;$ (iv) $\partial b(\theta) /\left.\partial \theta^{\prime}\right|_{\theta=\theta_{0}}$ is full column rank; $(v) \Omega_{T} \stackrel{p}{\rightarrow} \Omega$, a positive definite matrix of finite constants; (vi) $Q_{T}^{I I}(\theta)$ converges uniformly to $k(\theta)^{\prime} \Omega k(\theta)$ on $\Theta$ where $k(\theta)=h_{0}-b(\theta)$; (vii) $M_{I T}(\theta)$ converges uniformly to $-\partial b(\theta) / \partial \theta^{\prime}$, a matrix of finite constants (possibly dependent on $\theta$ ) on $N_{\theta, \epsilon}$. 
Assumption 8(i) states that the simulator is the true distribution for $X$. Assumption 8(i) combined with Assumption 6(i)-(ii) imply that $h_{0}=b\left(\theta_{0}\right)$. This property combined with Assumption 8 (ii) yields the global identification on the binding function discussed in Section 2. Assumption 8 (iv) is the first-order local identification condition associated with the binding function that is also discussed in Section 2. Note that these identification conditions imply $\ell \geq p$. Assumption 8(v)-(vi) specialize Assumptions 2(i) and 2(iii) to the II estimator considered here. Assumptions 8(ii) and $8(\mathrm{v})$ and $h_{0}=b\left(\theta_{0}\right)$ are equivalent to Assumption 2(v) and so it follows from Proposition 1 that $\hat{\theta}_{I I} \stackrel{p}{\rightarrow} \theta_{0}$ under Assumptions 6 and 8 .

Since $\theta_{0}$ is interior to $\Theta$, the indirect estimator solves with probability approaching 1 the firstorder condition $(9)$ :

$$
M_{I T}\left(\hat{\theta}_{I I}\right)^{\prime} \Omega_{T} m_{I T}\left(\hat{\theta}_{I I}\right)=0 .
$$

By a first-order mean value expansion of $m_{I T}$ around $\theta_{0}$, we have:

$$
M_{I T}\left(\hat{\theta}_{I I}\right) \Omega_{T}\left(m_{I T}\left(\theta_{0}\right)+M_{I T}\left(\dot{\theta}_{T}\right)\left(\hat{\theta}_{I I}-\theta_{0}\right)\right)=0
$$

with $\dot{\theta}_{T} \in\left(\hat{\theta}_{I I}, \theta_{0}\right)$ and may differ from row to row. We deduce that:

$$
\hat{\theta}_{I I}-\theta_{0}=\dot{F}_{T}\left(h_{T}-\frac{1}{s} \sum_{i=1}^{s} h_{T}^{(i)}\left(\theta_{0}\right)\right),
$$

with

$$
\dot{F}_{T}=-\left(M_{I T}\left(\hat{\theta}_{I I}\right)^{\prime} \Omega_{T} M_{I T}\left(\dot{\theta}_{T}\right)\right)^{-1} M_{I T}\left(\hat{\theta}_{I I}\right)^{\prime} \Omega_{T}
$$

The asymptotic distribution of $\hat{\theta}_{I I}-\theta_{0}$ depends on that of $h_{T}-\frac{1}{s} \sum_{i=1}^{s} h_{T}^{(i)}\left(\theta_{0}\right)$. Define $B_{T}$ to be the diagonal $\ell \times \ell$ matrix of rates of convergence with all its diagonal elements equal to $\sqrt{T}$ except for the last one which is $T^{1 / 4}$. Under Assumption 6, we can apply Theorem 1 to deduce that,

$$
B_{T}\left(h_{T}-h_{0}\right) \stackrel{d}{\rightarrow} \mathbb{X}, \quad \text { and } \quad B_{T}\left(h_{T}^{(i)}-h_{0}\right) \stackrel{d}{\rightarrow} \mathbb{X},
$$

for all $i=1, \ldots, s$, where $\mathbb{X}$ is defined as in Theorem $1(\mathrm{c})$ only with $m^{(a)}(\cdot)$, its derivatives and $h$ replacing respectively $m(\cdot)$, its analogous derivatives and $\phi$. Hence, assuming that $h_{T}^{(i)}\left(\theta_{0}\right)$ are independent across $i$ and independent of $h_{T}^{22}$, we have:

$$
B_{T}\left(h_{T}-\frac{1}{s} \sum_{i=1}^{s} h_{T}^{(i)}\left(\theta_{0}\right)\right) \stackrel{d}{\rightarrow} \mathbb{Y} \equiv \mathbb{X}_{0}-\frac{1}{s} \sum_{i=1}^{s} \mathbb{X}_{i}
$$

where $\mathbb{X}_{0}, \mathbb{X}_{1}, \ldots \mathbb{X}_{s}$ are independent with the same distribution as $\mathbb{X}$.

The fact that the rates of convergence in the diagonal of $B_{T}$ are not all equal make the determination of the rate of convergence of $\hat{\theta}_{I I}-\theta_{0}$ from that of $m_{I T}\left(\theta_{0}\right)$ more complicated than in the standard case. Pre-multiplying $(31)$ by $T^{1 / 4}$, we have:

$$
T^{1 / 4}\left(\hat{\theta}_{I I}-\theta_{0}\right)=\dot{F}_{T, \bullet \ell} T^{1 / 4} m_{I T, \ell}\left(\theta_{0}\right)+o_{P}(1)=F_{\bullet \ell} T^{1 / 4} m_{I T, \ell}\left(\theta_{0}\right)+o_{P}(1),
$$

${ }^{22}$ This is the case when there are no state variables so that the simulated samples are independent across $i=1, \ldots, s$. (See Gourieroux, Monfort, and Renault, 1993). 
where under Assumptions 6 and 8,

$$
\dot{F}_{T} \stackrel{p}{\rightarrow} F=\left\{\left(\frac{\partial b\left(\theta_{0}\right)}{\partial \theta^{\prime}}\right)^{\prime} \Omega\left(\frac{\partial b\left(\theta_{0}\right)}{\partial \theta^{\prime}}\right)\right\}^{-1}\left(\frac{\partial b\left(\theta_{0}\right)}{\partial \theta^{\prime}}\right)^{\prime} \Omega,
$$

$\partial b\left(\theta_{0}\right) / \partial \theta^{\prime}=\partial b(\theta) /\left.\partial \theta^{\prime}\right|_{\theta=\theta_{0}}$, and $\dot{F}_{T, \bullet \ell}$ and $F_{\bullet \ell}$ are the $\ell^{t h}$ column of $\dot{F}_{T}$ and $F$, respectively. Hence:

$$
T^{1 / 4}\left(\hat{\theta}_{I I}-\theta_{0}\right) \stackrel{d}{\rightarrow} F_{\bullet} \mathbb{Y}_{\ell}
$$

where $\mathbb{Y}_{\ell}$ is the $\ell^{\text {th }}$ component of $\mathbb{Y}$.

This asymptotic distribution represents a $p$-dimensional sample dependent random vector that converges in distribution to a random vector that has only one dimension of randomness. In fact, $T^{1 / 4}$ appears to be the slowest rate of convergence of $\left(\hat{\theta}_{I I}-\theta_{0}\right)$ in any direction in the space. However asymptotic inference on $\theta_{0}$ would benefit from a further characterization of the asymptotic distribution. We expect that some linear combinations of $\hat{\theta}_{I I}-\theta_{0}$ converge faster than others that converge at the rate $T^{1 / 4}$.

To derive this asymptotic distribution, we rely on a second-order expansion of $m_{I T}\left(\hat{\theta}_{I I}\right)$ around $\theta_{0}$. Such a higher order expansion is required by the fact that $\left(\hat{\theta}_{I I}-\theta_{0}\right)$ has the rate of convergence $T^{1 / 4}$ in some directions and therefore, its quadratic function is a non-negligible component of $m_{I T}\left(\hat{\theta}_{I I}\right)$. We make the following assumption.

Assumption 9. $\Delta_{I T, k}(\theta) \equiv \frac{\partial^{2} m_{I T, k}(\theta)}{\partial \theta \partial \theta^{\prime}}$ converges in probability uniformly over $N_{\theta, \epsilon}$ to $\Delta_{I, k}(\theta) \equiv$ $-\frac{\partial^{2} b_{k}(\theta)}{\partial \theta \partial \theta^{\prime}}$ for $k=1, \ldots, \ell$ and $b_{k}(\theta)$ denotes the $k^{\text {th }}$ element of $b(\theta)$.

By a second-order mean value expansion of $m_{I T}\left(\theta_{0}\right)$ around $\hat{\theta}_{I I}$, and after re-arranging, we have:

$$
m_{I T}\left(\hat{\theta}_{I I}\right)=m_{I T}\left(\theta_{0}\right)+M_{I T}\left(\hat{\theta}_{I I}\right)\left(\hat{\theta}_{I I}-\theta_{0}\right)-\frac{1}{2}\left(\left(\hat{\theta}_{I I}-\theta_{0}\right)^{\prime} \Delta_{I T, k}\left(\dot{\theta}_{T}\right)\left(\hat{\theta}_{I I}-\theta_{0}\right)\right)_{1 \leq k \leq \ell},
$$

where $\dot{\theta}_{T} \in\left(\theta_{0}, \hat{\theta}_{I I}\right)$ may differ from row to row. Solving this in $\left(\hat{\theta}_{I I}-\theta_{0}\right)$ yields:

$$
\hat{\theta}_{I I}-\theta_{0}=\hat{F}_{T}\left(m_{I T}\left(\theta_{0}\right)-\frac{1}{2}\left(\left(\hat{\theta}_{I I}-\theta_{0}\right)^{\prime} \Delta_{I T, k}\left(\dot{\theta}_{T}\right)\left(\hat{\theta}_{I I}-\theta_{0}\right)\right)_{1 \leq k \leq \ell}\right),
$$

with

$$
\hat{F}_{T}=-\left(M_{I T}\left(\hat{\theta}_{I I}\right)^{\prime} \Omega_{T} M_{I T}\left(\hat{\theta}_{I I}\right)\right)^{-1} M_{I T}\left(\hat{\theta}_{I I}\right)^{\prime} \Omega_{T} .
$$

To characterize the directions of fast convergence of $\hat{\theta}_{I I}-\theta_{0}$, let $\hat{S}$ be the $p \times p$ matrix with unit and pairwise orthogonal $p$-vectors as rows with the last row equal to the last column of $\hat{F}_{T}$ normalized and let $\hat{S}_{1}$ be the $(p-1) \times p$ sub-matrix of the first $(p-1)$ rows of $\hat{S}$. Remark 4 below gives how the matrix $\hat{S}$ can be determined as a continuous function of the last column of $\hat{F}_{T}$.

Remark 4. Let $\left(e_{1}, \ldots, e_{p}\right)$ be the canonical basis of $\mathbb{R}^{p}$, with $e_{j}$ the p-vector of 0's except for its $j^{\text {th }}$ entry equal 1. Consider Condition C below:

Condition $C: \quad u \in \mathbb{R}^{p} \backslash\{0\}$ and there exists a unique subset $\left\{e_{i_{1}}, \ldots, e_{i_{p-1}}\right\}$ of $p-1$ elements of the canonical basis of $\mathbb{R}^{p}$ such that the absolute determinant of the $(p \times p)$-matrix $\left(\begin{array}{llll}u & e_{i_{1}} & \ldots & e_{i_{p-1}}\end{array}\right)$ is maximum. 
Let $u \in \mathbb{R}^{p} \backslash\{0\}$. Consider the following three step procedure. Step 1: Find $p-1$ elements of the canonical basis of $\mathbb{R}^{p}$ such that the absolute determinant of $\left(u e_{i_{1}} \ldots e_{i_{p-1}}\right)$ is maximum, with $i_{k}$ an increasing sequence in $k$. Step 2: Determine an orthonormal basis from $\left(\begin{array}{llll}u & e_{i_{1}} & \ldots & e_{i_{p-1}}\end{array}\right)$ using the Gram-Schmidt orthonormalization process and let $\left(\tilde{u}, \tilde{e}_{1}, \tilde{e}_{2}, \ldots, \tilde{e}_{p-1}\right)$ be the resulting orthonormal basis. Step 3: Take

$$
S(u)=\left(\begin{array}{lllll}
\tilde{e}_{1} & \tilde{e}_{2} & \ldots & \tilde{e}_{p-1} & \tilde{u}
\end{array}\right)^{\prime} .
$$

We can show that if $u$ satisfies Condition $C$, then $u \mapsto S(u)$ is continuous at $u$. In particular, if $F_{\bullet \ell}$ satisfies Condition $C$, we set $\hat{S}=S\left(\hat{F}_{T, \bullet \ell}\right)$. The continuity of $S$ at $F_{\bullet \ell}$ allows the application of the continuous mapping theorem as we do in the proof of Theorem 2 below.

If $p-1$ elements $\left\{e_{i_{1}}^{f}, \ldots, e_{i_{p-1}}^{f}\right\}$ of the canonical basis of $\mathbb{R}^{p}$ are known to be linearly independent of $F_{\bullet \ell}$, then one can skip Step 1 in the definition of $S(u)$ and use $\left(e_{i_{1}}^{f}, \ldots, e_{i_{p-1}}^{f}\right)$ in Step 2. In this case, $F_{\bullet \ell}$ need not satisfy Condition $C$.

By construction, $\hat{S}_{1} \hat{F}_{T} m_{I T}\left(\theta_{0}\right)$ does not depend on the slow converging component, $m_{I T, \ell}\left(\theta_{0}\right)$, of $m_{I T}\left(\theta_{0}\right)$. We therefore have:

$$
\sqrt{T} \hat{S}_{1}\left(\hat{\theta}_{I I}-\theta_{0}\right)=\hat{S}_{1} \hat{F}_{T} B_{T}\left(m_{I T}\left(\theta_{0}\right)-\frac{1}{2}\left(\left(\hat{\theta}_{I I}-\theta_{0}\right)^{\prime} \Delta_{I T, k}\left(\dot{\theta}_{T}\right)\left(\hat{\theta}_{I I}-\theta_{0}\right)\right)_{1 \leq k \leq \ell}\right) .
$$

Combining (32) and (34) and letting $S$ be the probability limit of $\hat{S}$ and $B_{I T}=\left(\begin{array}{cc}\sqrt{T} I_{p-1} & 0 \\ 0 & T^{1 / 4}\end{array}\right)$, we obtain the following result.

Theorem 2. Let the II estimator be defined by (30) with the target function in (29), and Assumptions 6-9 hold. If the $s$ indirect inference samples are generated independently and the last column of $F$ satisfies Condition $C$ in Remark 4 then:

$$
B_{I T} \hat{S}\left(\hat{\theta}_{I I}-\theta_{0}\right) \stackrel{d}{\rightarrow}\left(\begin{array}{c}
S_{1} F\left(\mathbb{Y}-\frac{\left(\mathbb{Y}_{\ell}\right)^{2}}{2}\left(F_{\bullet \ell}^{\prime} \Delta_{I, k}\left(\theta_{0}\right) F_{\bullet \ell}\right)_{1 \leq k \leq \ell}\right) \\
S_{p \bullet} F_{\bullet \ell} \mathbb{Y}_{\ell}
\end{array}\right),
$$

where $S_{1}$ is the sub-matrix of the first $(p-1)$ rows of $S, S_{p}$ is the $p^{\text {th }}$ row of $S, \mathbb{Y}=\mathbb{X}_{0}-\frac{1}{s} \sum_{i=1}^{s} \mathbb{X}_{i}$, with $\mathbb{X}_{j}$ 's independently and identically distributed as $\mathbb{X}$, and $\mathbb{Y}_{\ell}$ is the $\ell^{\text {th }}$ element of $\mathbb{Y}$.

The proof is relegated to the Appendix. The asymptotic distribution of $B_{I T} \hat{S}\left(\hat{\theta}_{I I}-\theta_{0}\right)$ can be simulated by replacing $S, F$ and $\Delta_{I, k}\left(\theta_{0}\right), k=1, \ldots, \ell$ by their estimates, $\hat{S}, \hat{F}$ and $\Delta_{I T, k}\left(\hat{\theta}_{I I}\right)$, $k=1, \ldots, \ell$. The simulation of $\mathbb{Y}$ is based on that of $\mathbb{X}$ which is described in the previous section.

Remark 5. In the case where the rank deficiency in the auxiliary model appears in a way that no column of the Jacobian matrix is nil, we can obtain the asymptotic distribution of the indirect estimator as follows. The asymptotic distribution of $B_{T} \hat{R}^{-1}\left(h_{T}-h_{0}\right)$ is derived in the previous section. Let $\widetilde{\mathbb{X}}$ denote this asymptotic distribution in either Case 1 or Case 2. From (31), we can show that:

$$
T^{1 / 4}\left(\hat{\theta}_{I I}-\theta_{0}\right)=F R_{\bullet \ell} T^{1 / 4}\left(\hat{R}^{-1} m_{I T}\left(\theta_{0}\right)\right)_{\ell}+o_{P}(1)
$$


where $R_{\bullet} \ell$ is the $\ell^{\text {th }}$ column of $R$ and $\left(\hat{R}^{-1} m_{I T}\left(\theta_{0}\right)\right)_{\ell}$ is the $\ell^{\text {th }}$ element of $\hat{R}^{-1} m_{I T}\left(\theta_{0}\right)$. Also, from (33), we have

$$
\hat{\theta}_{I I}-\theta_{0}=\hat{F}_{T} \hat{R}\left(\hat{R}^{-1} m_{I T}\left(\theta_{0}\right)-\frac{1}{2} \hat{R}^{-1}\left(\left(\hat{\theta}_{I I}-\theta_{0}\right)^{\prime} \Delta_{I T, k}\left(\dot{\theta}_{T}\right)\left(\hat{\theta}_{I I}-\theta_{0}\right)\right)_{1 \leq k \leq \ell}\right) .
$$

Letting $\hat{S}_{R}$ be row-wise, the orthonormal basis obtained by completing the $\ell^{\text {th }}$ column of $\hat{F}_{T} \hat{R}$ according to Remark 4 , and $S_{R}$ its probability limit, we have that:

$$
B_{I T} \hat{S}_{R}\left(\hat{\theta}_{I I}-\theta_{0}\right) \stackrel{d}{\rightarrow}\left(\begin{array}{c}
S_{R, 1} F R\left(\tilde{\mathbb{Y}}-\frac{\left(\tilde{\mathbb{Y}}_{\ell}\right)^{2}}{2} R^{-1}\left(R_{\bullet \ell}^{\prime} F^{\prime} \Delta_{I, k}\left(\theta_{0}\right) F R \bullet \ell\right)_{1 \leq k \leq \ell}\right) \\
S_{R, p \bullet} F R_{\bullet \ell} \tilde{\mathbb{Y}}_{\ell}
\end{array}\right),
$$

where $\tilde{\mathbb{Y}}=\tilde{\mathbb{X}}_{0}-\frac{1}{s} \sum_{i=1}^{s} \tilde{\mathbb{X}}_{i}$, with $\tilde{\mathbb{X}}_{j}$ 's are independent and identically distributed as $\tilde{\mathbb{X}}$ and $S_{R, 1}$, $S_{R, p \bullet}$ are defined similarly to $S_{1}$ and $S_{p}$ in Theorem 2.

\subsection{The local identification condition associated with the binding func- tion holds at second order}

We now consider the large sample behaviour of the II estimator when the binding function satisfies the global identification condition but only satisfies the local identification condition at second order. For this analysis, it is assumed that the auxiliary parameter vector is globally and first-order locally identified.

Assumption 10. (i) Assumption 6(i)-(ii) hold; (ii) $m_{T}^{(a)}(h)$ is differentiable in an $\epsilon$-neighbourhood of $h_{0}, N_{h, \epsilon}$, and the derivatives of $m_{T}^{(a)}(h)$ converge uniformly over $N_{h, \epsilon}$ to those of $m^{(a)}(h)$; (iii) $\operatorname{rank}\left\{M^{(a)}\left(h_{0}\right)\right\}=\ell$.

To present the necessary regularity conditions on the binding function, we introduce the following notation. We partition the parameter vector as $\theta=\left(\theta_{1: p-1}^{\prime}, \theta_{p}\right)^{\prime}$ where $\theta_{p}$ is the $p^{t h}$ element of $\theta$ but for ease of notation in the formulae below set $\theta_{1}=\theta_{1: p-1}$. Define $M^{(b)}\left(\theta_{0}\right)=-\partial b(\theta) /\left.\partial \theta^{\prime}\right|_{\theta=\theta_{0}}$ and $G^{(b)}=-\partial^{2} b(\theta) /\left.\partial \theta_{p}^{2}\right|_{\theta=\theta_{0}}$. Here the superscript " $(b)$ " indicates quantities pertaining to the binding function.

Assumption 11. (i) Assumption 8(i)-(ii) hold; (ii) $b(\theta)$ is continuous on $\Theta$ and three times continuously differentiable on $N_{\theta, \epsilon}$, an $\epsilon$-neighbourhood of $\theta_{0}$; (iii) $M^{(b)}\left(\theta_{0}\right)=\left[D^{(b)}, 0_{\ell \times 1}\right]$ where $D^{(b)}$ is $\ell \times(p-1)$ matrix of constants with full column rank; $(i v) G^{(b)}$ is a vector of finite constants with Rank $\left\{\left[D^{(b)}, G^{(b)}\right]\right\}=p$; (v) Assumption 8(v)-(vi) hold; (vi) $m_{I T}(\theta)$ is three times continuously differentiable on $N_{\theta, \epsilon}$ and the derivatives of $m_{I T}(\theta)$ converge in probability uniformly over $N_{\theta, \epsilon}$ to those of $-b(\theta)$.

The combination of Assumptions 10 and 11 has important implications for the source of the firstorder local identification failure in the binding function as we now explain. Within our framework, the binding function is defined as

$$
b(\theta)=\operatorname{argmin}_{h} E_{\theta}[g(X(\theta), h)]^{\prime} W E_{\theta}[g(X(\theta), h)] .
$$


Using Assumption 10, we can equivalently characterize binding function through the first order conditions:

$$
b(\theta)=h \text { where } h \text { satisfies }\left\{E_{\theta}\left[\frac{\partial g(X(\theta), h)}{\partial h^{\prime}}\right]\right\}^{\prime} W E_{\theta}[g(X(\theta), h)]=0 .
$$

From the Implicit Function Theorem, Dhrymes (1984)[Proposition 94, p.109] and using (28), we have:

$$
\frac{\partial b\left(\theta_{0}\right)}{\partial \theta^{\prime}}=-\left[M^{(a)^{\prime}} W M^{(a)}\right]^{-1} M^{(a)^{\prime}} W \Gamma_{\theta} .
$$

where $M^{(a)}=M^{(a)}\left(h_{0}\right)$ and

$$
\Gamma_{\theta}=E_{\theta_{0}}\left[\left.\frac{\partial g\left(X(\theta), h_{0}\right)}{\partial \theta^{\prime}}\right|_{\theta=\theta_{0}}\right] .
$$

From Assumption 10, $\operatorname{rank}\left\{M^{(a)^{\prime}} W\right\}=\ell$ and so rank deficiency in $\partial b\left(\theta_{0}\right) / \partial \theta^{\prime}$ can occur in two ways. ${ }^{23}$ First, we have $\operatorname{rank}\left\{\Gamma_{\theta}\right\}=p$ but $\operatorname{rank}\left\{M^{(a)^{\prime}} W \Gamma_{\theta}\right\}<p$ : this situation can be interpreted as one in which the identification failure is due to the choice of target function rather than the auxiliary model. In this case, it may be possible to restore first-order identification by use of a different target function. Second, we have $\operatorname{rank}\left\{\Gamma_{\theta}\right\}<p$ : in this case the identification failure stems from the choice of auxiliary model. ${ }^{24}$ Our analysis covers both possibilities; we return to this issue in Section 5.3.

The asymptotic distribution of the II estimator is readily available by similar arguments to the proof of Theorem 1. In the scenario here, the limiting distribution is a function of the limiting distribution of $\left[m_{I T}\left(\theta_{0}\right)^{\prime}, \partial m_{I T}\left(\theta_{0}\right) / \partial \theta_{p}\right]$. Below we make an assumption about the large sample behaviour of this term, but before doing so provide a motivation for the condition in question. Within our framework here, we have:

$$
\sqrt{T} m_{I T}\left(\theta_{0}\right)=\sqrt{T}\left(h_{T}-h_{0}\right)-\frac{1}{s} \sum_{i=1}^{s} \sqrt{T}\left(h_{T}^{(i)}\left(\theta_{0}\right)-h_{0}\right)
$$

Under Assumption 10 and some additional mild conditions, ${ }^{25} \sqrt{T}\left(h_{T}-h_{0}\right)$ converges in distribution to a random vector $Z_{0 h}$ with $Z_{0 h} \sim N\left(0, V_{h}\right)$ where $V_{h}$ is defined by the right-hand side of (10) with $M^{(a)}$ replacing $M\left(\phi_{0}\right)$ and $V_{m}$ defined as $V_{m}=\lim _{T \rightarrow \infty} \operatorname{Var}\left(\sqrt{T} m_{T}^{(a)}\left(h_{0}\right)\right)$. It then follows that

$$
\sqrt{T} m_{I T}\left(\theta_{0}\right) \stackrel{d}{\rightarrow} \mathbb{Z}_{0 h}(s) \equiv Z_{0 h}^{(0)}+\frac{1}{s} \sum_{i=1}^{s} Z_{0 h}^{(i)}
$$

where $Z_{0 h}^{(k)}(k=0, \ldots, s)$ are independent copies of $Z_{0 h}$. Using Assumption 11(iii), it follows from a Central Limit Theorem that

$$
T^{1 / 2} \partial m_{I T}\left(\theta_{0}\right) / \partial \theta_{p} \stackrel{d}{\rightarrow} \mathbb{Z}_{1 h}(s) \equiv \frac{1}{s} \sum_{i=1}^{s} Z_{1 h}^{(i)}
$$

\footnotetext{
${ }^{23}$ For the following discussion, recall $M^{(a)^{\prime}} W$ is $\ell \times q$ and $\Gamma_{\theta}$ is $q \times p$ with $q \geq \ell \geq p$, and $\operatorname{rank}\{A B\} \leq$ $\min (\operatorname{rank}\{A\}, \operatorname{rank}\{B\})$ for conformable matrices $A$ and $B$.

${ }^{24}$ We note that if the target function consists of a set of raw data moments then local identification can only fail because $\operatorname{rank}\left\{\Gamma_{\theta}\right\}<p$.

${ }^{25}$ See Proposition 2 in Section 2.
} 
where $Z_{1 h}^{(k)}(k=1, \ldots, s)$ are independent copies of $Z_{1 h} \sim N\left(0, V_{1}\right)$, and using (37), we have

$$
V_{1}=\left[M^{(a)^{\prime}} W M^{(a)}\right]^{-1} M^{(a)^{\prime}} W V_{2} W M^{(a)}\left[M^{(a)^{\prime}} W M^{(a)}\right]^{-1},
$$

with

$$
V_{2}=\lim _{T \rightarrow \infty} \operatorname{Var}\left[T^{-1 / 2} \sum_{t=1}^{T} \partial g\left(X\left(\theta_{0}\right), h_{0}\right) / \partial \theta_{p}\right] .
$$

This limiting behaviour is implied by the following assumption.

Assumption 12. (i) The auxiliary model (28) is such that: $h_{0}=b\left(\theta_{0}\right), \sqrt{T}\left(h_{T}-h_{0}\right)$ converges in distribution to $Z_{0 h} \sim N\left(0, V_{h}\right)$ and

$$
\sqrt{T}\left(\begin{array}{c}
m_{I T}\left(\theta_{0}\right) \\
\frac{\partial m_{I T}\left(\theta_{0}\right)}{\partial \theta_{p}}
\end{array}\right) \stackrel{d}{\rightarrow}\left(\begin{array}{c}
\mathbb{Z}_{0 h}(s) \\
\mathbb{Z}_{1 h}(s)
\end{array}\right) .
$$

for $\mathbb{Z}_{0 h}(s)$ and $\mathbb{Z}_{1 h}(s)$ defined above. (ii) $\Omega_{T}-\Omega=o_{P}\left(T^{-1 / 4}\right)$, $\frac{\partial m_{I T}}{\partial \theta_{1}^{\prime}}\left(\theta_{0}\right)-D^{(b)}=O_{P}\left(T^{-1 / 2}\right)$, $\frac{\partial^{2} m_{I T}}{\partial \theta_{p}^{2}}\left(\theta_{0}\right)-G^{(b)}=O_{P}\left(T^{-1 / 2}\right), \quad \frac{\partial^{2} m_{I T}}{\partial \theta_{1}^{2} \partial \theta_{p}}\left(\theta_{0}\right)-G_{1 p}^{(b)}=o_{P}(1), \quad$ and $\quad \frac{\partial^{3} m_{I T}}{\partial \theta_{p}^{3}}\left(\theta_{0}\right)-L^{(b)}=o_{P}(1)$, with $G_{1 p}^{(b)}=-\frac{\partial^{2} b}{\partial \theta_{1}^{\prime} \partial \theta_{p}}\left(\theta_{0}\right) \quad$ and $\quad L^{(b)}=-\frac{\partial^{3} b}{\partial \theta_{p}^{3}}\left(\theta_{0}\right)$.

Let $M_{d}^{(b)}, P_{g}^{(b)}, M_{d}^{(b)} g, \mathbb{R}_{1}^{(b)}(s), \sigma_{G}^{(b)}$, and $H^{(b)}$ be defined as $M_{d}, P_{g}, M_{d g}, \mathbb{R}_{1}(s), \sigma_{G}$, and $H$, respectively with $I_{q}, D, G, W, \mathbb{Z}_{0}, \mathbb{Z}_{1}, L$ and $G_{1 p_{\phi}}$ replaced by $I_{\ell}, D^{(b)}, G^{(b)}, \Omega, \mathbb{Z}_{0 h}(s), \mathbb{Z}_{1 h}(s)$, $L^{(b)}$ and $G_{1 p}^{(b)}$, respectively.

Theorem 3. Under Assumptions 11 and 12, we have

(a)

$$
\sqrt{T}\left(\begin{array}{c}
\hat{\theta}_{1}-\theta_{0,1} \\
\left(\hat{\theta}_{p}-\theta_{0, p}\right)^{2}
\end{array}\right) \stackrel{d}{\rightarrow}\left(\begin{array}{c}
H^{(b)} \mathbb{Z}_{0 h}(s)+H^{(b)} G^{(b)} \mathbb{V}^{(b)}(s) / 2 \\
\mathbb{V}^{(b)}(s)
\end{array}\right),
$$

with $\mathbb{V}^{(b)}(s)=-2 \frac{\left.\mathbb{Z}^{(b)}(s) \mathbb{(} \mathbb{Z}^{(b)}(s)<0\right)}{\sigma_{G}^{(b)}}$ and $\mathbb{Z}^{(b)}(s)=G^{(b) \prime} \Omega^{1 / 2} M_{d}^{(b)} \Omega^{1 / 2} \mathbb{Z}_{0 h}(s)$.

(b) If in addition, $\mathbb{R}_{1}^{(b)}(s)$ does not have an atom of probability at 0 , then:

$$
\left(\begin{array}{c}
\sqrt{T}\left(\hat{\theta}_{1}-\theta_{0,1}\right) \\
T^{1 / 4}\left(\hat{\theta}_{p}-\theta_{0, p}\right)
\end{array}\right) \stackrel{d}{\rightarrow} \mathbb{X}(s) \equiv\left(\begin{array}{c}
H^{(b)} \mathbb{Z}_{0 h}(s)+H^{(b)} G^{(b)} \mathbb{V}^{(b)}(s) / 2 \\
(-1)^{\mathbb{B}_{s}} \sqrt{\mathbb{V}^{(b)}(s)}
\end{array}\right)
$$

with $\mathbb{B}_{s}=\mathbb{I}\left(\mathbb{R}_{1}^{(b)}(s) \geq 0\right)$.

This result follows readily from Theorem 1 . As with that earlier result, the limiting distribution is non-standard but it can easily be simulated using similar approach as that described in Remark 2 . It is worth mentioning that part (a) of the theorem does not require the whole set of Assumptions 11 and 12. Indeed, (a) holds if we maintain twice continuous differentiability in Assumptions 11(ii) and 11 (vi) in addition to Assumption 12(i) with merely $\sqrt{T} m_{I T}\left(\theta_{0}\right) \stackrel{d}{\rightarrow} \mathbb{Z}_{0 h}(s)$; without imposing the joint convergence in distribution. Assumption 12(ii) and third order differentiability are useful for the higher order expansion of the $Q_{T}^{I I}(\theta)$ from which is deduced the sign of $T^{1 / 4}\left(\hat{\theta}_{p}-\theta_{0, p}\right)$.

If the Jacobian matrix of the binding function at $\theta_{0}$ is of rank $p-1$ but does not have a column of zeros, similar transformations as those leading to (22) and (24) can be made for the characterization of the asymptotic distribution of the indirect inference estimator. 


\subsection{Optimal (based on first order asymptotics) II estimation}

In our analysis, we consider the framework where the target function is the GMM estimator of $h_{0}$ based on (28). While this choice of target function has a certain practical appeal as GMM is widely implemented in empirical economics, Frazier and Renault (2016) show that this choice of target function is not optimal for II estimation under the assumptions that permit a first-order asymptotic analysis. The latter conditions include first-order local identification involving both the auxiliary parameter vector and the binding function. Below we consider the properties of this optimal estimator within our framework in which local identification only holds at second order. To do so, we must briefly summarize Frazier and Renault's results within the standard first-order asymptotic framework.

Frazier and Renault (2016) consider the case where the auxiliary model is (28) and the target function is the Method of Moments (MM) estimator of $h_{0}$ based on the moment conditions:

$$
A E\left[g\left(X, h_{0}\right)\right]=A m^{(a)}\left(h_{0}\right)=0,
$$

where $A$ is a $\ell \times q$ matrix of given constants with full row rank and (28) is assumed to first-order locally identify $h_{0}$ that is, $M^{(a)}$ is full column rank. Let $h_{T}(A)$ denote the MM estimator of $h_{0}$ based on (38). As pointed out by Frazier and Renault (2016), this framework includes an estimator that is first-order equivalent to the two-step GMM estimator based on (28) by setting $A=M^{(a) \prime} V^{-1}$ where $V$ is $A \operatorname{var}\left[m_{T}^{(a)}\left(h_{0}\right)\right]=\lim _{T \rightarrow \infty} \operatorname{Var}\left[T^{1 / 2} m_{T}^{(a)}\left(h_{0}\right)\right] \cdot{ }^{26}$ Let $b_{A}(\theta)$ denote the binding function associated with the use of $h_{T}(A)$ as the target function. Frazier and Renault (2016) show that for a given $A$ the optimal choice of weighting matrix in the II estimation of $\theta_{0}$ is:

$$
\Omega^{*}(A)=\left\{\operatorname{Avar}\left[h_{T}(A)-h_{0}\right]\right\}^{-1}=M^{(a) \prime} A^{\prime}\left\{A V A^{\prime}\right\}^{-1} A M^{(a)} .
$$

Let $\hat{\theta}_{T}(A)$ be the II estimator defined by (30) with the binding function $b_{A}(\theta)$ and a weighting matrix that satisfies $\Omega_{T} \stackrel{p}{\rightarrow} \Omega^{*}(A)$. Frazier and Renault (2016) show that the variance of the limiting distribution of $T^{1 / 2}\left(\hat{\theta}_{T}(A)-\theta_{0}\right)$ is:

$$
\operatorname{Avar}\left[\hat{\theta}_{T}(A)-\theta_{0}\right]=\left(\left\{\frac{\partial b_{A}\left(\theta_{0}\right)}{\partial \theta^{\prime}}\right\}^{\prime}\left\{\operatorname{Avar}\left[h_{T}(A)-h_{0}\right]\right\}^{-1}\left\{\frac{\partial b_{A}\left(\theta_{0}\right)}{\partial \theta^{\prime}}\right\}\right)^{-1}
$$

where

$$
\frac{\partial b_{A}\left(\theta_{0}\right)}{\partial \theta^{\prime}}=-\left[A M^{(a)}\right]^{-1} A \Gamma_{\theta}
$$

Using the latter result, Frazier and Renault (2016) establish that the efficiency bound for estimation (in this way) of $\theta_{0}$ is $\left(\Gamma_{\theta}^{\prime} V^{-1} \Gamma_{\theta}\right)^{-1}$ and that this can be achieved by setting $A=A^{*}$ where

$$
A^{*}=\left(\begin{array}{c}
\Gamma_{\theta}^{\prime} V^{-1} \\
\cdots \\
C^{\prime}
\end{array}\right)
$$

and $C$ is an arbitrary matrix of dimension $q \times(\ell-p)$ whose columns do not belong to the space spanned by the columns of $V^{-1} \Gamma_{\theta}$. As Frazier and Renault (2016) show, the use of the two-step

\footnotetext{
${ }^{26}$ This choice of $A$ yields an estimator that is only first-order asymptotically equivalent to the GMM estimator because $A$ is treated as given whereas the first order conditions of GMM estimation acknowledge the dependence of the Jacobian matrix on $h$.
} 
GMM estimator of $h_{0}$ based on (28) as the target function only achieves this bound if $V^{-1 / 2} \Gamma_{\theta}$ lies in the column space of $V^{-1 / 2} M^{(a)}$. This leads to an important insight about Indirect Inference estimation, namely that efficient estimation of $h_{0}$ does not necessarily translate into efficient estimation of $\theta_{0}$ in this context.

We now consider the large sample properties of $\hat{\theta}_{T}(A)$ within the frameworks of Section 5.1 and 5.2 above. First, as in Section 5.1, we consider the case in which the binding function satisfies the global and first-order local identification conditions and the auxiliary model satisfies the global identification but only satisfies the local identification condition at second order. For ease of exposition, we assume the auxiliary model exhibits that pattern of identification in Assumption 6 .

If the auxiliary parameters are only identified locally at second order then the consistency of $\hat{\theta}_{T}(A)$ can no longer be established via the conventional arguments. The reason is that $\Omega^{*}(A)$ in (39) is singular for any choice of $A$ because $M^{(a)}$ is rank deficient. Therefore, even though the binding function satisfies the global identification condition, the weighting matrix, $\Omega^{*}(A)$, does not exhibit the positive definiteness required for the population analog to the II minimand to have a unique minimum at $\theta_{0}$. Thus the standard proof of consistency of the II estimator does not go through. Following some proofs of the consistency of GMM-type estimators, ${ }^{27}$ consistency can still be established if $\Omega^{*}(A)$ is positive semi-definite and $\Omega^{*}(A)\left\{h_{0}-b_{A}(\theta)\right\}=0 \Leftrightarrow \theta=\theta_{0}$. However, given (39), the latter condition requires that $h_{0}-b_{A}(\theta)$ does not lie in the null space of $M^{(a)}$ for any $\theta \neq \theta_{0}$ which is a strong assumption that - it seems to us - would be hard to justify in many circumstances. $^{28}$

Even if $\hat{\theta}_{T}(A)$ can be argued to be consistent then it does not have the first-order asymptotic distribution on which the optimality of $A^{*}$ is based because if $m^{(a)}\left(h_{0}\right)=0$ only identifies $h_{0}$ locally at second order then $A m^{(a)}\left(h_{0}\right)=0$ does not identify $h_{0}$ locally at first order. Recalling $\operatorname{rank}(A)=\ell$ and Assumption $6(\mathrm{iii})$, this can be seen by noting that

$$
\frac{\partial A m^{(a)}\left(h_{0}\right)}{\partial h^{\prime}}=A M^{(a)}=\left[A D^{(a)}, 0_{q \times 1}\right]
$$

and so $\operatorname{rank}\left\{A M^{(a)}\right\} \leq \ell-1$. If $\operatorname{rank}\left\{A M^{(a)}\right\}=\ell-1$ and the second-order local identification conditions hold ${ }^{29}$ the large sample distribution theory of the Method of Moments estimator based on $A m^{(a)}\left(h_{0}\right)=0$ is characterized by Theorem 1(c). ${ }^{30}$ Hence the large sample distribution of $\hat{\theta}_{T}(A)$ is given by Theorem 2 (with $A m_{T}^{(a)}(\cdot)$ replacing $m_{T}^{(a)}(\cdot)$ etc.).

We now consider the large sample properties of $\hat{\theta}_{T}(A)$ within the framework of Section 5.2 in which the binding function satisfies the global identification condition but only satisfies the local identification at second order, and the auxiliary model satisfies both global and first-order local identification conditions. For an arbitrary full row rank matrix $A$, it follows from (40) (and similar arguments to Section 5.2) that the first-order identification failure can arise in principle in two ways: $^{31}$ (i) $\operatorname{rank}\left\{\Gamma_{\theta}\right\}=p$ but $\operatorname{rank}\left\{A \Gamma_{\theta}\right\}<p$; (ii) $\operatorname{rank}\left\{\Gamma_{\theta}\right\}<p$. However if we restrict attention to $A=A^{*}$ then it can be seen from (41) that if $\operatorname{rank}\left\{\Gamma_{\theta}\right\}=p$ then $\operatorname{rank}\left\{A^{*} \Gamma_{\theta}\right\}=p$ by construction. Thus, by using $\hat{\theta}_{T}\left(A^{*}\right)$, one possible source of first-order local identification failure in the binding function is avoided; and if $\operatorname{rank}\left\{\Gamma_{\theta}\right\}=p$ then $\hat{\theta}_{T}\left(A^{*}\right)$ has the first-order asymptotic distribution

\footnotetext{
${ }^{27}$ For example see Newey and McFadden (1984) [p.2132].

${ }^{28}$ For example, under Assumption 6(iii), this condition would imply that $h_{0}-b_{A}(\theta) \not \subset\left(0_{1 \times(\ell-1)}^{\prime}, 1\right)^{\prime}$ for any $\theta$.

${ }^{29}$ Since $\partial^{2} A m^{(a)}\left(h_{0}\right) / \partial h_{\ell}^{2}=A G$ the condition for second-order local identification is $\operatorname{rank}\left\{A\left[D^{(a)}, G^{(a)}\right]\right\}=\ell$.

${ }^{30}$ This characterization involves replacing $m^{(a)}(\cdot)$ and its relevant derivatives by $A m^{(a)}(\cdot)$ and the its analogous derivatives.

${ }^{31}$ This statement is premised on $A M^{(a)}$ being nonsingular.
} 
described above. However, if the failure in first-order local identification is due to $\operatorname{rank}\left\{\Gamma_{\theta}\right\}<p$ then the limiting distribution of $\hat{\theta}_{T}\left(A^{*}\right)$ can be obtained from Theorem 3 subject to the model satisfying the assumptions of this result.

Following the approach of Frazier and Renault (2016), it would be interesting to try to characterize an optimal combination of $A$ and $\Omega$ that minimizes the asymptotic variance of $\hat{\theta}_{T}(A)$ within the frameworks of either Sections 5.1 or 5.2. However, the nature of the limiting distribution in Theorems 2 and 3 make the asymptotic variances of the II estimator depend non-trivially on $A$ and $\Omega$, making the determination of their optimal values a hard problem to solve analytically. Such an analysis is beyond the scope of this paper and left to future research.

\section{Monte Carlo results}

This section illustrates the finite sample performance some of the asymptotic results derived in this paper through Monte Carlo simulations. We are mainly concerned with coverage probabilities of confidence intervals (CI) based on the asymptotic distribution derived for the GMM and II estimators in Theorems 1 and 2, respectively.

Our simulations are based on the dynamic panel data model in Example 1 in Section 3. The simulated data are generated from $\varepsilon_{i t} \sim \operatorname{NID}\left(0, \sigma_{\varepsilon}^{2}\right), i=1, \ldots, n, t=1,2$ independent of $\left(\eta_{i}, y_{i 0}\right) \sim$ $N I D(0, \Sigma)$, with $\Sigma_{11}=\sigma_{\eta}^{2}, \Sigma_{22}=\sigma_{0}^{2}$, and $\Sigma_{12}=\sigma_{0 \eta}$. The autoregressive dynamic in (12) is then used to obtain samples $\left\{y_{i}=\left(y_{i 1}, y_{i 2}\right): i=1, \ldots, n\right\}$ for various values of $\rho$.

The parameter of interest $\rho$ is estimated by 2 -step GMM and II using the moment restriction in (14). The 2-step GMM estimator $\hat{\rho}$ is obtained using the identity matrix as weighting matrix at the first step and the estimated optimal weighting matrix at the second step. We obtain the indirect inference estimator as follows. We fix the indirect inference sample parameters at $\tilde{\sigma}_{0}^{2}, \tilde{\sigma}_{\eta}^{2}, \tilde{\sigma}_{0 \eta}$, and $\tilde{\sigma}_{\varepsilon}^{2}$. Then, for each $\rho, s$ samples of size $n:\left\{y_{i}^{k}(\rho): i=1, \ldots, n\right\}(k=1, \ldots, s)$ are simulated and the 2-step GMM estimator of $\rho, h_{k}(\rho)$, is obtained for each sample $k=1, \ldots, s$. The estimated binding function $b_{s}(\rho)=\frac{1}{s} \sum_{k=1}^{s} h_{k}(\rho)$ is used to determine the II estimator $\hat{\rho}^{I I}$ of $\rho$ :

$$
\hat{\rho}^{I I}=\arg \min _{\rho}\left|\hat{\rho}-b_{s}(\rho)\right|^{2} .
$$

We follow Gourieroux, Phillips, and Yu (2010) by setting $\tilde{\sigma}_{0}^{2}, \tilde{\sigma}_{\eta}^{2}, \tilde{\sigma}_{0 \eta}$, and $\tilde{\sigma}_{\varepsilon}^{2}$ to the set of values $\sigma_{0}^{2}$, $\sigma_{\eta}^{2}, \sigma_{0 \eta}$, and $\sigma_{\varepsilon}^{2}$ that govern the dynamics of the original sample. We later relax this for robustness checking. We set $s=50$ throughout.

One of the main interests in the II estimator, as established by Gourieroux, Phillips, and Yu (2010), is its ability to reduce potential finite sample bias from the original estimator. However, if interest lies with CI's, in the case where $\rho=1$ and $\sigma_{\eta}^{2}=\sigma_{0 \eta}=0$, the standard asymptotic distribution fails and inference must be based on Theorem 2 .

In each of our Monte Carlo experiments, CI's from the standard theory and that from our theory are considered. For the GMM and as already mentioned (see Remark 3), when the moment condition model has a single parameter that is not locally identified at the first order but rather at the second order, the asymptotic distribution of $n^{1 / 4}(\hat{\rho}-\rho)$ is a simple function of a Gaussian variable and CI's can be derived analytically using quantiles from the standard Gaussian distribution. However, in general, the asymptotic distribution of $n^{1 / 4}(\tilde{\rho}-\rho)$ (with $\tilde{\rho}$ being the GMM or II estimator) can be simulated and one can consider symmetric CI's based on the quantiles of the asymptotic distribution of $n^{1 / 4}(\tilde{\rho}-\rho)$ or the so-called equal-tailed CI's that use $\alpha / 2$-quantile and $(1-\alpha / 2)$-quantile of this 
asymptotic distribution. Throughout this section, only results from symmetric CI's are reported. Equal-tailed CI's have very similar performance and have not been reported. Simulated quantiles are obtained from 1,000 draws from the estimated asymptotic distribution of $n^{1 / 4}(\tilde{\rho}-\rho)$ where $G$ and $W$ are replaced by their estimates.

Table 1 gives the results related to GMM estimation and coverage rate of CI's based on GMM using the standard asymptotics (Cov- 1 ) and our results (Cov-2 and Cov- 3 using analytic and simulated quantiles, respectively). We take $\sigma_{0}^{2}=\sigma_{\varepsilon}^{2}=1$ and $\sigma_{\eta}^{2}=\sigma_{0 \eta}=0$ and consider $\rho=0.2,0.3,0.5,0.75,0.8,0.9,0.95,0.97,0.98,1.0,1.1,1.2,1.3$ and 1.5. Even though first-order local identification issues arise at $\rho=1.0$, this range of values for $\rho$ allows us to investigate the finite sample performance of the non-standard CI near $\rho=1.0$, i.e. near singularity of the Jacobian matrix of the moment function. The Euclidean norm of the simulated mean of this Jacobian, $D(\rho)$ in (42), and the second derivative matrix, $G(\rho)$ in (43), are also reported as $|\overline{\hat{D}}|$ and $|\overline{\hat{G}}|$ respectively.

First, we observe that Cov-2 and Cov-3 have approximately the same values meaning that the non-standard CI's based on simulation or on quantiles from the standard normal distribution are almost identical. Besides, for values of $\rho$ 'far' from the singularity point $(\rho=0.2,0.3,0.5,1.3$ and 1.5), the coverage rates of the standard CI (Cov-1) seem to converge to the nominal level $95 \%$ as $n$ becomes large whereas the non-standard CI substantially over-covers at those values for $n$ large with coverage rates larger than $99 \%$.

However, near $\rho=1.0$, the non-standard CI has coverage rates of about nominal $95 \%$ while the standard CI substantially under-covers at around $82 \%$ for $n=5,000$. Specifically, the standard CI performs poorly for $\rho$ ranging from 0.8 through 1.2 even in large samples. In small samples ( $n=50,100,200)$, except for $\rho=1.3$ and 1.5, the non-standard CI seems to outperform the standard one as it delivers coverage rates substantially closer to nominal. It is worth mentioning that the smaller the Jacobian norm $(|\overline{\hat{D}}|)$, the better the non-standard CI performs.

Figure 1 reports, for $n=5,000$, histograms of the simulated GMM estimators for $\rho=0.3,1.0,1.3$ and also QQ-plots of these distributions against the standard normal distribution. These reveal that the GMM estimator has a very different distribution for $\rho=1$, the point of first-order identification failure than at the other two points at which $\rho$ is first-order locally identified. The distribution for $\rho=1$ is also evidently non-normal.

To explore the behaviour of the estimator in a different neighbourhood to the point of first-order local identification failure, we fix $\rho=1$ and set $\sigma_{0 \eta}=\lambda, \sigma_{\eta}^{2}=|\lambda|$, with $\lambda=0, \pm 0.1,0.2,0.3,0.5$. These results are reported in Table 2. Qualitatively, the results are the same as in Table 1: the CI's based on Theorem 1 have approximately the nominal coverage level for $\lambda$ values close to 0 , the point of first-order local identification failure but the coverage is too high outside this neighbourhood. In contrast, the coverage of the CI based on the standard theory is well below the nominal $95 \%$ level in this neighbourhood: for example at $n=5,000$, the coverage is between $78 \%, 82 \%$ and $84 \%$ for $\lambda=-0.1,0,0.1$, respectively.

Table 3 reports analogous results for II to those for Table 1 for GMM. These results indicate that the CI's based on standard asymptotic theory are too low at and in the neighbourhood of the point of first-order local identification failure whereas the coverage for the CI's based on Theorem 2 are closer to the nominal level although only achieve the nominal level at the largest sample size. Comparing the GMM and II CI's based on our theory for parameter values in the neighbourhood of the point of first-order local identification failure, it can be seen that the coverage rates for GMM tend to be closer to the nominal level than those for II. As noted above, one reason for employing II is bias reduction. In Table 3 we report the simulated bias and RMSE of the two estimators. From Table 3 , it can be seen that for $\rho \leq 1$, our II estimator exhibits lower bias; but for $\rho>1$ GMM 
exhibits less bias.

The simulated distribution of the II estimator is displayed by Figure 2 which is the II analogue of Figure 1. We can see that at singularity $(\rho=1)$, II is also clearly non-normal whereas as $\rho=0.3$ or 1.3, related histograms and QQ-plots reveal a behaviour of II in line with normality.

We conclude this simulation experiments by investigating the robustness of the properties of the II estimator. In this experiment, we assume the researcher uses (13) as the auxiliary model but calibrates the value of $\theta_{2}$. So for the true data generation process: $\rho=1, \sigma_{\varepsilon}^{2}=\sigma_{0}^{2}=1$ and $\sigma_{0 \eta}=\sigma_{\eta}^{2}=0$; but the calibrated values of $\theta_{2}$ are: $\tilde{\sigma}_{\varepsilon}^{2}=\tilde{\sigma}_{0}^{2}=1, \tilde{\sigma}_{0 \eta}=\lambda$ and $\tilde{\sigma}_{\eta}^{2}=|\lambda|$, $\lambda=0, \pm 0.1,0.2$. Note that due to the calibration, only $\rho$ is estimated via II. The results are displayed in Table 4. The CI based on Theorem 2 outperforms the standard CI for all values of $\lambda$ and for all the sample sizes considered. We can also see that for $n=5,000$, the coverage rates from the non-standard CI are all close to nominal except for $\lambda=-0.1$ where the coverage rate is $80.1 \%$. Note that this still outperforms the standard CI by about 4 percentage points. Besides, for each sample size, we measure the stability of coverage across $\lambda$ 's by the mean absolute deviation (MAD) from the nominal level of $95 \%$. Using this metric, we can see that the non-standard CI has a better robustness property since its MAD varies from $13.5(n=200)$ to $3.36(n=5,000)$ and is always smaller than that of the standard CI which lies between 10.3 and 20.5. It is also worth mentioning that the bias reduction property expected for II is also robust to the deviations considered for the II samples since this estimator has a smaller bias than GMM across $\lambda$ 's.

\section{Concluding remarks}

In this paper, we provide new results on the limiting behaviour of GMM and II estimators when local identification conditions fail at first-order but hold at second order. For our analysis of II, we focus on the case where the auxiliary model consists of a set of population moment conditions indexed by a vector of auxiliary parameters and the target function for the II estimation is a GMM estimator of the auxiliary parameter vector. Within this framework, the standard first-order asymptotic theory is premised on the assumption that the binding function satisfies both global and first-order local identification conditions and the auxiliary parameters are globally and firstorder locally identified within the auxiliary model. We present the limiting distributions of the II estimator in the case where the local identification condition for either the binding function or the auxiliary only holds at second order. These limiting distributions are different in each case, and, like the limiting distribution of the GMM estimator, non-standard. However, we show that these limit distributions can be easily simulated making it possible to perform inference about the parameters in these settings. We also use our results to analyze the large sample behaviour under second-order local identification of a version of II that has recently been shown by Frazier and Renault (2016) to be optimal within the standard first-order asymptotic framework. We find that the large sample properties of this optimal estimator are affected by the failure of the first-order local identification involving either the binding function or the auxiliary model. However, interestingly, we find that use of the optimal estimator means inferences are robust to one possible source of failure of the first-order local identification condition involving the binding function.

While first-order local identification may only fail at a point in the parameter space, our simulation results indicate that our theory based on second-order identification can provide a better approximation to finite sample behaviour of GMM and II estimators than standard first order asymptotic theory in a neighbourhood of the point of first-order local identification failure. Our simulation study further reveals that the limiting distribution theory derived in our paper leads 
to reliable GMM/II-based inferences in moderate to large samples in the neighbourhood of the point of first-order identification failure. Comparing GMM and II, we find our limiting distribution theory provides a reasonable approximation to the behaviour of the GMM at smaller sample sizes than it does for the II estimator, but that II exhibits smaller bias at the point of first-order local identification failure.

The choice of limit theory then requires knowledge of the quality of the identification but this may be difficult to assess a priori. It would be interesting to explore diagnostics for cases when local identification fails at first order but not at second order. Such diagnostics for local identification have recently been receiving some attention in the context of DSGE models. Iskrev (2010) and $\mathrm{Qu}$ and Tkachenko (2012) develop methods for evaluating the first-order local identification based on numerical evaluation of the Jacobian over the parameter space. An attractive feature of such analyses is that they can reveal areas of the parameter space where first-order identification fails. By their nature, these methods focus on first-order identification. However, we conjecture that, given the complexity of the models and the need for approximations to their solutions, parameters of DSGE models may be second-order but not first-order locally identified in some cases of interest. The results presented in our paper provide a basis for performing inference about the parameters in this context. It would therefore be interesting to explore extensions of these diagnostics to look for evidence of second-order local identification.

Alternatively, it may be of interest to explore ways to generate confidence sets based on GMM and II estimators that are robust to first- or second- order identification. One possible approach may be the use of bootstrap methods, building from recent work on bootstrapping the GMM overidentification test by Dovonon and Gonçalves (2016). 


\section{References}

Andrews, D. W. K. (1991). 'Heteroscedasticity and autocorrelation consistent covariance matrix estimation', Econometrica, 59: 817-858.

Arellano, M., and Bond, S. R. (1991). 'Some tests of specification for panel data: Monte Carlo evidence and an application to employment equations', Review of Economic Studies, 58: 277 297.

Arellano, M., Hansen, L. P., and Sentana, E. (2012). 'Underidentification?', Journal of Econometrics, 138: 256-280.

Azzalini, A. (2005). 'The skew-normal distribution and related multivariate families', Scandanavian Journal of Statistics, 32: 159-188.

Barankin, E., and Gurland, J. (1951). 'On asymptotically normal efficient estimators: I', University of California Publications in Statistics, 1: 86-130.

Blundell, R., and Bond, S. R. (1998). 'Initial conditions and moment restrictions in dynamic panel data models', Journal of Econometrics, 87: 115-143.

Canova, F., and Sala, L. (2009). 'Back to square one: identification issues in DSGE models', Journal of Monetary Economics, 56: 431-449.

Christiano, L., Eichenbaum, M., and Evans, C. (2005). 'Nominal rigidities and the dynamic effects of a shock to monetary policy', Journal of Political Economy, 113: 1-45.

Coenen, G., Levin, A. T., and Christoffel, K. (2007). 'Identifying the influences of nominal and real rigidities in aggregate price-setting behavior', Journal of Monetray Economics, 54: 2439-2466.

Dhrymes, P. J. (1984). Mathematics for econometrics. Springer Verlag, New York, NY, U. S. A., second edn.

Diebold, F. X., and Nerlove, M. (1989). 'The dynamics of exchange rate volatility: a multivariate latent factor ARCH model', Journal of Applied Econometrics, 4: 1-22.

Dovonon, P., and Gonçalves, S. (2016). 'Bootstrapping the GMM overidentification test under firstorder underidentification', Discussion paper, Department of Economics, Concordia University, Montreal, Canada.

Dovonon, P., and Hall, A. R. (2015). 'GMM and Indirect Inference - an appraisal of their connections and new results on their properties under second order identification', Discussion paper, Department of Economics, University of Manchester, Discussion paper EDP-1505.

Dovonon, P., and Renault, E. (2009). 'GMM overidentification test with first order underidentification', Discussion paper, Department of Economics, Concordia University, Montreal, Canada. 2586 .

(2013). 'Testing for common conditionally heteroscedastic factors', Econometrica, 81: 2561- 
Doz, C., and Renault, E. (2004). 'Conditionally heteroskedastic factor models: Identification and instrumental variables estimation', Discussion paper, University Cergy-Pontoise, THEMA, France, 2004-13.

(2006). 'Factor volatility in mean models: a GMM approach', Econometric Reviews, 25: 275-309.

Duffie, D., and Singleton, K. J. (1993). 'Testing for common conditionally heteroscedastic factors', Econometrica, 61: 929-952.

Dufour, J.-M. (1997). 'Some impossibility theorems in econometrics with applications to structural and dynamic models', Econometrica, 65: 1365-1387.

Dufour, J.-M., Khalaf, L., and Kichian, M. (2013). 'Identification-robust analysis of DSGE and structural macroeconomic models', Journal of Monetary Economics, 60: 340-350.

Dupaigne, M., Fève, P., and Matheron, J. (2007). 'Avoiding pitfalls in using structural VARs to estimate economic models', Review of Economic Dynamics, 10: 238-255.

Ferguson, T. S. (1958). 'A method of generating best asymptotically normal estimates with application to the estimation of bacterial densities', Annals of Mathematical Statistics, 29: 1046-1062.

Fiorentini, G., Sentana, E., and Shephard, N. (2004). 'Likelihood-based estimation of generalised ARCH structures', Econometrica, 72: 1481-1517.

Frazier, D. T., and Renault, E. (2016). 'Indirect inference with(out) constraints', Discussion paper, Department of Economics, University of Econometrics and Business Statistics, Monash University, Melbourne, New South Wales, Australia.

Gallant, A. R., and Tauchen, G. (1996). 'Which moments to match?', Econometric Theory, 12: $657-681$

Garcia, R., Renault, E., and Veredas, D. (2011). 'Estimation of stable distributions by indirect inference', Journal of Econometrics, 161: 325-337.

Ghysels, E., and Guay, A. (2003). 'Structural change tests for simulated method of moments', Journal of Econometrics, 115: 91-123.

Gourieroux, C., Monfort, A., and Renault, E. (1993). 'Indirect inference', Journal of Applied Econometrics, 8: S85-S118.

Gourieroux, C., Phillips, P. C. B., and Yu, J. (2010). 'Indirect Inference for dynamic panel models', Journal of Econometrics, 157: 68-77.

Hall, A. R. (2015). 'Econometricians have their moments: GMM at 32', Economic Record, 91, S1: $1-24$.

Hamilton, J. D. (1994). Time series analysis. Princeton University Press, Princeton, NJ, U. S. A.

Hansen, L. P. (1982). 'Large sample properties of Generalized Method of Moments estimators', Econometrica, 50: 1029-1054. 
Hansen, L. P., and Singleton, K. S. (1982). 'Generalized instrumental variables estimation of nonlinear rational expectations models', Econometrica, 50: 1269-1286.

Heaton, J. (1995). 'An empirical investigation of asset pricing with temporally dependent preference specifications', Econometrica, 63: 681-717.

Iskrev, N. (2010). 'Local identification in DSGE models', Journal of Monetary Economics, 57: 189-202.

Jansen, I., Hens, N., Molenberghs, G., Aerts, M., Verbeke, G., and Kenward, M. G. (2006). 'The nature of sensitivity in monotone missing not at random models', Computational Statistics and Data Analysis, 50: 830-858.

Kleibergen, F. (2005). 'Testing parameters in GMM without assuming that they are ideintified', Econometrica, 73: 1103-1124.

Komunjer, I., and Ng, S. (2011). 'Dynamic identification of dynamic stochastic general equilibrium models', Econometrica, 79: 1995-2032.

Kruiniger, H. (2013). 'Quasi ML estimation of the panel AR(1) model with arbitrary initial conditions', Journal of Econometrics, 173: 175-188.

(2014). 'A further look at Modied ML estimation of the panel AR(1) model with xed eects and arbitrary initial conditions', Discussion paper, University of Durham, unpublished mimeo.

Le, V. P. M., Meenagh, D., Minford, P., and Wickens, M. (2011). 'How much nominal rigidity is there in the US economy? Testing a new Keynesian DSGE model using indirect inference', Journal of Economic Dynamics and Control, 35: 2078-2104.

Lee, J. H., and Liao, Z. (2016). 'On Standard Inference for GMM with Local Identication Failure of Known Forms', Discussion paper, unpublished mimeo, Department of Economics, University of Illinois, Urbana IL, USA.

Madsen, E. (2009). 'GMM-based inference in the $\mathrm{AR}(1)$ panel data model for parameter values where local idntification fails', Discussion paper, Centre for Applied Microeconometrics, Department of Economics, University of Copenhagen, Copenhagen, Denmark.

McFadden, D. (1989). 'A method of simulated moments for estimation of discrete response models without numerical integration', Econometrica, 57: 995-1026.

Mutschler, W. (2015). 'Identification of DSGE models - the effect of higher-order approximation and pruning', Journal of Economic Dynamics and Control, 56: 34-54.

Newey, W. K., and McFadden, D. L. (1994). 'Large sample estimation and hypothesis testing', in R. Engle and D. L. McFadden (eds.), Handbook of Econometrics, vol. 4, pp. 2113-2247. Elsevier Science Publishers, Amsterdam, The Netherlands.

Newey, W. K., and Smith, R. J. (2004). 'Higher order properties of GMM and generalized empirical likelihood estimators', Econometrica, 72: 219-256. 
Neyman, J. (1949). 'Contribution to the theory of the $\chi^{2}$ test', in Proceedings of the Berkeley Symposium on Mathematical Statistics and Probability, pp. 239-273. University of California Press, Berkeley, CA, USA.

Neyman, J., and Pearson, E. S. (1928). 'On the use and interpretation of certain test criteria for purposes of statistical inference: part II', Biometrika, 20A: 263-294.

Pearson, K. S. (1894). 'Contributions to the mathematical theory of evolution', Philosophical transactions of the Royal Society of London (A), 185: 71-110.

(1895). 'Contributions to the mathematical theory of evolution, II: skew variation', Philosophical transactions of the Royal Society of London (A), 186: 343-414.

$\mathrm{Qu}$, Z. (2014). 'Inference in dynamic stochastic general equilibrium models with possible weak identification', Quantitative Economics, 3: 95-132.

Qu, Z., and Tkachenko, D. (2012). 'Identification and frequency domain quasi-mximum likelihood estimation of linearized dynamic stochastic general equilibrium models', Quantitative Economics, 3: $95-132$.

Rotnitzky, A., Cox, D. R., Bottai, M., and Robins, J. (2000). 'Likelihood-based inference with singular information matrix', Bernouilli, 6: 243-284.

Ruge-Murcia, F. J. (2007). 'Methods to estimate dynamic stochastic general equilibrium models', Journal of Economic Dynamics and Control, 31: 2599-2636.

Sargan, J. D. (1983). 'Identification and lack of identification', Econometrica, 51: 1605-1633.

Sentana, E. (2015). 'Finite Underidentification', Discussion paper, CEMFI Working Paper 1508, Madrid, Spain.

Smith, A. A. (1993). 'Estimating nonlinear time series models using simulated vector autoregressions', Journal of Applied Econometrics, 8: S63-S84.

Staiger, D., and Stock, J. (1997). 'Instrumental variables regression with weak instruments', Econometrica, 65: 557-586.

Stingo, F. C., Stanghellini, E., and Capobianco, R. (2011). 'On the estimation of a binary response model in a selected population', Journal of Statistical Planning and Inference, 141: 3293-3303.

Stock, J., and Wright, J. (2000). 'GMM with weak identification', Econometrica, 68: 1055-1096.

The Royal Swedish Academy of Sciences (2013). Prizes in Economic Sciences 2013, Scientific Background. http://www.nobelprize.org/nobel_prizes/economic-sciences/laureates/2013/advancedeconomicsciences2013.pdf.

Wright, J. H. (2003). 'Detecting Lack of Identification in GMM', Econometric Theory, 19: 322-330. 


\section{A Examples from Section 3}

Example 1: panel data model

The moment condition in (13) can be derived using the assumptions in the text along with the following equation, implied by (12), that holds for all $i=1, \ldots, n$ and $t=1,2$ :

$$
y_{i t}=\rho^{t} y_{i 0}+\sum_{s=0}^{t-1} \rho^{s} \eta_{i}+\sum_{s=1}^{t} \rho^{t-s} \varepsilon_{i s} .
$$

It can be shown that the moment condition in (13) globally identify $\theta$ if the data generating process is such that the true parameter value $\theta^{*}$ satisfies $\sigma_{0 \eta}^{*} \neq\left(1-\rho^{*}\right) \sigma_{0}^{*^{2}}$. Nevertheless, (13) also ensures global identification of $\theta$ if $\rho^{*}=1$ and $\sigma_{0 \eta}^{*}=\sigma_{\eta}^{*}=0$; that is when the $\operatorname{AR}(1)$ panel dynamics has unit root and no fixed effects.

The Jacobian matrix of this moment function is:

$$
\left(\begin{array}{cc}
-\frac{\partial H(\rho)}{\partial \rho} \theta_{2} & -H(\rho))
\end{array}\right.
$$

As shown by Madsen (2009), see also Dovonon and Gonçalves (2015), if $\rho^{*}=1$ and $\sigma_{0 \eta}^{*}=\sigma_{\eta}^{*^{2}}=0$, this Jacobian matrix has rank $4<5$ at the true parameter value so that the moment condition model (13) is first-order locally under identified. In fact, it can be seen that $H(\rho)$ is of rank 4 for any $\rho$ and $\frac{\partial H(1)}{\partial \rho} \theta_{2}^{*}=H(1) \delta$ with $\delta=\left(0, \sigma_{\varepsilon}^{*^{2}}, \sigma_{0}^{*^{2}},-\sigma_{\varepsilon}^{*^{2}}\right)^{\prime}$.

The statements about the identification of $\rho$ based on (14) can be justified as follows. It can be shown that (14) globally identifies $\rho$ so long as (13) globally identifies $\theta$ (see above). The Jacobian matrix associated with these moment conditions is:

$$
D(\rho)=-\left(H_{1, \rho}^{(1)}-H_{1, \rho} H_{2, \rho}^{-1} H_{2, \rho}^{(1)}\right) \theta_{2}(\rho)
$$

where $H_{k, \rho}=H_{k}(\rho)$ and $H_{k, \rho}^{(j)}=\frac{\partial^{j} H_{k}(\rho)}{\partial \rho^{j}}$. Since $\frac{\partial H(1)}{\partial \rho} \theta_{2}^{*}=H(1) \delta$, we have: $D(1)=0$. Some straightforward calculations show that the second-order derivative of the moment function in (14) is:

$$
G(\rho)=-\left(H_{1, \rho}^{(2)}-H_{1, \rho} H_{2, \rho}^{-1} H_{2, \rho}^{(2)}\right) \theta_{2}(\rho)+2\left(H_{1, \rho}^{(1)}-H_{1, \rho} H_{2, \rho}^{-1} H_{2, \rho}^{(1)}\right) H_{2, \rho}^{-1} H_{2, \rho}^{(1)} \theta_{2}(\rho),
$$

and $G(1) \neq 0$ in general.

\section{Example 2: a conditional heteroscedastic factor model}

Auxiliary Model: There exists $\delta$ such that $\left(\begin{array}{ll}1 & -\delta\end{array}\right)\left(\begin{array}{c}\gamma_{1} \\ \gamma_{2}\end{array}\right)=0$. Hence, $y_{1 t}-\delta y_{2 t}=u_{1 t}-\delta u_{2 t}$. We therefore have:

$$
E\left[\left(y_{1 t}-\delta y_{2 t}\right)^{2} \mid \mathfrak{F}_{t-1}\right]=c\left(=\Omega_{1}+\delta^{2} \Omega_{2}\right) .
$$

Taking an instrument $z_{t-1}$ from $\mathfrak{F}_{t-1}$ such that $\operatorname{Cov}\left[z_{t-1}, y_{2 t}^{2}\right] \neq 0$ and $E\left[z_{t-1}\right] \neq 0$, (e.g lagged square returns), we have:

$$
m_{0}\left(z_{t-1}, y_{t}, \delta, c\right)=0
$$

with $m_{0}\left(z_{t-1}, y_{t}, \delta, c\right)=E\left[\left(\begin{array}{c}1 \\ z_{t-1}\end{array}\right)\left\{\left(y_{1 t}-\delta y_{2 t}\right)^{2}-c\right\}\right]$. 
We can show that this model identifies globally both $\delta$ and $c$. We also have:

$$
E\left[y_{1 t}^{2}\right]=\gamma_{1}^{2}+\Omega_{1} \equiv b_{1}, \quad E\left[y_{1 t}^{2}\right]=\gamma_{1}^{2}+\Omega_{2} \equiv b_{2}, \quad \text { and } \quad E\left[y_{1 t} y_{1, t-1}\right]=\gamma_{1} \gamma_{2} \equiv b_{3} .
$$

The auxiliary model is defined as:

$$
\begin{aligned}
m_{0}\left(z_{t-1}, y_{t}, \delta, c\right) & =0 \\
E\left[y_{1 t}^{2}\right] & =b_{1} \\
E\left[y_{2 t}^{2}\right] & =b_{2} \\
E\left[y_{1 t} y_{2 t}\right] & =b_{3} .
\end{aligned}
$$

The parameter vector $h=\left(b_{1}, b_{2}, b_{3}, \delta, c\right)^{\prime}$ of this model is globally identified. In addition, the parameter $\theta$ of the structural model can be determined from $h$. In fact, we can use the relations:

$$
b_{1}=\gamma_{1}^{2}+\Omega_{1}, \quad b_{2}=\gamma_{2}^{2}+\Omega_{2}, \quad b_{3}=\gamma_{1} \gamma_{2}, \quad c=\Omega_{1}+\delta^{2} \Omega_{2}, \quad \text { and } \quad c=b_{1}+\delta^{2} b_{2}-2 \delta b_{3}
$$

to obtain:

$$
\theta_{1} \equiv \gamma_{1}=\sqrt{\delta b_{3}}, \quad \theta_{2} \equiv \gamma_{2}=\sqrt{\frac{b_{3}}{\delta}}, \quad \theta_{3} \equiv \Omega_{1}=b_{1}-\delta b_{3}, \quad \theta_{4} \equiv \Omega_{2}=b_{2}-\frac{b_{3}}{\delta} .
$$

The auxiliary model is first-order locally underidentified: The Jacobian matrix of

$$
m_{0}\left(z_{t-1}, y_{t}, \delta, c\right)
$$

at the true parameter value is:

$$
-2 E\left[\left(\begin{array}{c}
1 \\
z_{t-1}
\end{array}\right) y_{2 t}\left(y_{1 t}-\delta y_{2 t}\right)\right]-\left(\begin{array}{c}
1 \\
E\left[z_{t}\right]
\end{array}\right) .
$$

At the true parameter value, $y_{1 t}-\delta y_{2 t}=u_{1 t}-\delta u_{2 t}$. Therefore, $E\left[y_{2 t}\left(y_{1 t}-\delta y_{2 t}\right) \mid \mathfrak{F}_{t-1}\right]=-\delta \Omega_{2}$. (Since $y_{2 t}\left(y_{1 t}-\delta y_{2 t}\right)=\gamma_{2} f_{t}\left(u_{1 t}-\delta u_{2 t}\right)+u_{2 t}\left(u_{1 t}-\delta u_{2 t}\right)$.) Thus, By the law of iterated expectations, this Jacobian matrix is:

$$
\left(2 \delta \Omega_{2}\left(\begin{array}{c}
1 \\
E\left[z_{t-1}\right]
\end{array}\right)-\left(\begin{array}{c}
1 \\
E\left[z_{t-1}\right]
\end{array}\right)\right)
$$

which is of rank 1 . In total, the Jacobian matrix of the auxiliary model is

$$
\left(\begin{array}{ccccc}
0 & 0 & 0 & 2 \delta \Omega_{2} & -1 \\
0 & 0 & 0 & 2 \delta \Omega_{2} E\left[z_{t-1}\right] & -E\left[z_{t-1}\right] \\
-1 & 0 & 0 & 0 & 0 \\
0 & -1 & 0 & 0 & 0 \\
0 & 0 & -1 & 0 & 0
\end{array}\right)
$$

which is of rank 4 instead of 5 .

The auxiliary model is second-order locally identified. To see this, we can check Condition (b) of Definition 1 by focusing solely on the first equality of $(44)$. Let $\phi=(\delta, c)^{\prime}$. The range space of $\frac{\partial m_{0}^{\prime}}{\partial \phi}\left(\phi_{0}\right)$ is determined by $u=a\left(2 \delta \Omega_{2},-1\right)^{\prime}: a \in \mathbb{R}$ and the null space of its transpose is determined by $v=b\left(E\left(z_{t-1}\right),-1\right)^{\prime}: b \in \mathbb{R}$. Also,

$$
\frac{\partial^{2} m_{0,1}}{\partial \delta^{2}} \equiv 2 E\left[y_{2 t}^{2}\right], \quad \frac{\partial^{2} m_{0,1}}{\partial \delta \partial c} \equiv 0, \quad \frac{\partial^{2} m_{0,1}}{\partial c^{2}} \equiv 0
$$


and

$$
\frac{\partial^{2} m_{0,2}}{\partial \delta^{2}} \equiv 2 E\left[z_{t-1} y_{2 t}^{2}\right], \quad \frac{\partial^{2} m_{0,2}}{\partial \delta \partial c} \equiv 0, \quad \frac{\partial^{2} m_{0,2}}{\partial c^{2}} \equiv 0
$$

Hence,

$$
\begin{aligned}
& \frac{\partial m_{0}}{\partial \phi^{\prime}}\left(\phi_{0}\right) u+\left(v^{\prime} \frac{\partial^{2} m_{0, k}}{\partial \phi \partial \phi^{\prime}} v\right)_{k=1,2} \\
= & \left(\begin{array}{c}
a\left(4 \delta^{2} \Omega_{2}^{2}+1\right)+2 b^{2}\left(E\left[z_{t-1}\right]\right)^{2} E\left[y_{2 t}^{2}\right] \\
a\left(4 \delta^{2} \Omega_{2}^{2}+1\right) E\left[z_{t-1}\right]+2 b^{2}\left(E\left[z_{t-1}\right]\right)^{2} E\left[y_{2 t}^{2} z_{t-1}\right]
\end{array}\right)
\end{aligned}
$$

which is equal to 0 if and only if $a=b=0$, i.e. $u=v=0$; so long as $\operatorname{Cov}\left[y_{2 t}^{2}, z_{t-1}\right] \neq 0$ and $E\left[z_{t-1}\right] \neq 0$.

\section{B Proofs}

Proof of Theorem 1 (a) We write $m_{T}(\hat{\phi})=m_{T}\left(\hat{\phi}_{1}, \hat{\phi}_{p_{\phi}}\right)$. A first-order mean value expansion of $\phi_{1} \mapsto$ $m_{T}\left(\phi_{1}, \hat{\phi}_{p_{\phi}}\right)$ around $\phi_{0,1}$ yields:

$$
m_{T}\left(\hat{\phi}_{1}, \hat{\phi}_{p_{\phi}}\right)=m_{T}\left(\phi_{0,1}, \hat{\phi}_{p_{\phi}}\right)+\frac{\partial m_{T}}{\partial \phi_{1}^{\prime}}\left(\bar{\phi}_{1}, \hat{\phi}_{p_{\phi}}\right)\left(\hat{\phi}_{1}-\phi_{0,1}\right),
$$

where $\bar{\phi}_{1} \in\left(\phi_{0,1}, \hat{\phi}_{1}\right)$ and may differ from row to row. Next, a second-order mean value expansion of $\phi_{p_{\phi}} \mapsto m_{T}\left(\phi_{0,1}, \phi_{p_{\phi}}\right)$ around $\phi_{0, p_{\phi}}$ that we plug back in the expression of $m_{T}(\hat{\phi})$ yields:

$$
\begin{aligned}
m_{T}(\hat{\phi})= & m_{T}\left(\phi_{0}\right)+\frac{\partial m_{T}}{\partial \phi_{1}^{\prime}}\left(\bar{\phi}_{1}, \hat{\phi}_{p_{\phi}}\right)\left(\hat{\phi}_{1}-\phi_{0,1}\right)+\frac{\partial m_{T}}{\partial \phi_{p_{\phi}}}\left(\phi_{0}\right)\left(\hat{\phi}_{p_{\phi}}-\phi_{0, p_{\phi}}\right) \\
& +\frac{1}{2} \frac{\partial^{2} m_{T}}{\partial \phi_{p_{\phi}}^{T}}\left(\phi_{0,1}, \bar{\phi}_{p_{\phi}}\right)\left(\hat{\phi}_{p_{\phi}}-\phi_{0, p_{\phi}}\right)^{2},
\end{aligned}
$$

where $\bar{\phi}_{p_{\phi}} \in\left(\phi_{0, p_{\phi}}, \hat{\phi}_{p_{\phi}}\right)$ and may differ from row to row.

Since $\frac{\partial m_{T}}{\partial \phi_{p_{\phi}}}\left(\phi_{0}\right)=O_{P}\left(T^{-1 / 2}\right)$ and $\hat{\phi}_{p_{\phi}}-\phi_{0, p_{\phi}}=o_{P}(1)$ (from Proposition 1), we have:

$$
\begin{aligned}
m_{T}(\hat{\phi})= & m_{T}\left(\phi_{0}\right)+\frac{\partial m_{T}}{\partial \phi_{1}^{\top}}\left(\bar{\phi}_{1}, \hat{\phi}_{p_{\phi}}\right)\left(\hat{\phi}_{1}-\phi_{0,1}\right) \\
& +\frac{1}{2} \frac{\partial^{2} m_{T}}{\partial \phi_{p_{\phi}}^{2}}\left(\phi_{0,1}, \bar{\phi}_{p_{\phi}}\right)\left(\hat{\phi}_{p_{\phi}}-\phi_{0, p_{\phi}}\right)^{2}+o_{P}\left(T^{-1 / 2}\right) .
\end{aligned}
$$

Let us define $\bar{D}=\frac{\partial m_{T}}{\partial \phi_{1}^{1}}\left(\bar{\phi}_{1}, \hat{\phi}_{p_{\phi}}\right)$ and $\bar{G}=\frac{\partial^{2} m_{T}}{\partial \phi_{p_{\phi}}^{2}}\left(\phi_{0,1}, \bar{\phi}_{p_{\phi}}\right)$. Pre-multiplying (45) by $\bar{D}^{\prime} W_{T}$, we get

$$
\begin{aligned}
\hat{\phi}_{1}-\phi_{0,1}= & \left(\bar{D}^{\prime} W_{T} \bar{D}\right)^{-1} \bar{D}^{\prime} W_{T}\left(m_{T}(\hat{\phi})-m_{T}\left(\phi_{0}\right)\right) \\
& -\frac{1}{2}\left(\bar{D}^{\prime} W_{T} \bar{D}\right)^{-1} \bar{D}^{\prime} W_{T} \bar{G}\left(\hat{\phi}_{p_{\phi}}-\phi_{0, p_{\phi}}\right)^{2}+o_{P}\left(T^{-1 / 2}\right) .
\end{aligned}
$$

The $o_{P}\left(T^{-1 / 2}\right)$ term stays with the same order because $\bar{D}$ and $W_{T}$ are both $O_{P}(1)$. Plugging this back into (45), we get:

$$
\begin{aligned}
m_{T}(\hat{\phi})= & m_{T}\left(\phi_{0}\right)+\bar{D}\left(\bar{D}^{\prime} W_{T} \bar{D}\right)^{-1} \bar{D}^{\prime} W_{T}\left(m_{T}(\hat{\phi})-m_{T}\left(\phi_{0}\right)\right) \\
& +\frac{1}{2} W_{T}^{-1 / 2} \bar{M}_{d} W_{T}^{1 / 2} \bar{G}\left(\hat{\phi}_{p_{\phi}}-\phi_{0, p_{\phi}}\right)^{2}+o_{P}\left(T^{-1 / 2}\right),
\end{aligned}
$$


with $\bar{M}_{d}=I_{q}-W_{T}^{1 / 2} \bar{D}\left(\bar{D}^{\prime} W_{T} \bar{D}\right)^{-1} \bar{D}^{\prime} W_{T}^{1 / 2}$.

Hence,

$$
\begin{aligned}
& m_{T}^{\prime}(\hat{\phi}) W_{T} m_{T}(\hat{\phi}) \\
& m_{T}^{\prime}\left(\phi_{0}\right) W_{T} m_{T}\left(\phi_{0}\right)+\frac{1}{4} \bar{G}^{\prime} W_{T}^{1 / 2} \bar{M}_{d} W_{T}^{1 / 2} \bar{G}\left(\hat{\phi}_{p_{\phi}}-\phi_{0, p_{\phi}}\right)^{4} \\
& +\left(\hat{\phi}_{p_{\phi}}-\phi_{0, p_{\phi}}\right)^{2} O_{P}\left(T^{-1 / 2}\right)+O_{P}\left(T^{-1}\right)
\end{aligned}
$$

The orders of magnitude in (47) follow from the fact that $\bar{M}_{d}$ converges in probability to $M_{d}$ and therefore is $O_{P}(1)$ and the fact that both $m_{T}\left(\phi_{0}\right)$ and $m_{T}(\hat{\phi})$ are $O_{P}\left(T^{-1 / 2}\right)$.

The latter comes from the fact that $m_{T}^{\prime}(\hat{\phi}) W_{T} m_{T}(\hat{\phi}) \leq m_{T}^{\prime}\left(\phi_{0}\right) W_{T} m_{T}\left(\phi_{0}\right)$ (by definition of GMM estimator). Since $W_{T}$ converges in probability to $W$ symmetric positive definite, we can claim that $m_{T}(\hat{\phi})$ is $O_{P}\left(T^{-1 / 2}\right)$ as is $m_{T}\left(\phi_{0}\right)$. Again, by the definition of the GMM estimator, the left hand side of (47) is less or equal to $m_{T}^{\prime}\left(\phi_{0}\right) W_{T} m_{T}\left(\phi_{0}\right)$ and this gives:

$$
\begin{aligned}
& \frac{1}{4} G^{\prime} W^{1 / 2} M_{d} W^{1 / 2} G T\left(\hat{\phi}_{p_{\phi}}-\phi_{0, p_{\phi}}\right)^{4}+o_{P}(1) T\left(\hat{\phi}_{p_{\phi}}-\phi_{0, p_{\phi}}\right)^{4} \\
\leq & O_{P}(1)+\sqrt{T}\left(\hat{\phi}_{p_{\phi}}-\phi_{0, p_{\phi}}\right)^{2} O_{P}(1)
\end{aligned}
$$

Thanks to Assumption 4(iii) and the fact that $W$ is nonsingular, $M_{d} W^{1 / 2} G \neq 0$. As a consequence, $G^{\prime} W^{1 / 2} M_{d} W^{1 / 2} G \neq 0$ which is sufficient to deduce from (48) that $T\left(\hat{\phi}_{p_{\phi}}-\phi_{0, p_{\phi}}\right)^{4}=O_{P}(1)$; or equivalently that $T^{1 / 4}\left(\hat{\phi}_{p_{\phi}}-\phi_{0, p_{\phi}}\right)=O_{P}(1)$. We obtain $\hat{\phi}_{1}-\phi_{0,1}=O_{P}\left(T^{-1 / 2}\right)$ from (46).

(b) From (a) and (45), we have

$$
m_{T}(\hat{\phi})=m_{T}\left(\phi_{0}\right)+D\left(\hat{\phi}_{1}-\phi_{0,1}\right)+\frac{1}{2} G\left(\hat{\phi}_{p_{\phi}}-\phi_{0, p_{\phi}}\right)^{2}+o_{P}\left(T^{-1 / 2}\right) .
$$

The first-order condition for interior solution is given by:

$$
\frac{\partial m_{T}}{\partial \phi^{\prime}}(\hat{\phi}) W_{T} m_{T}(\hat{\phi})=0 .
$$

In the direction of $\phi_{1}$, this amounts to

$$
\left(D^{\prime}+o_{P}(1)\right) W\left(\sqrt{T} m_{T}\left(\phi_{0}\right)+D \sqrt{T}\left(\hat{\phi}_{1}-\phi_{0,1}\right)+\frac{1}{2} G \sqrt{T}\left(\hat{\phi}_{p_{\phi}}-\phi_{0, p_{\phi}}\right)^{2}+o_{P}(1)\right)=0 .
$$

This gives:

$$
\sqrt{T}\left(\hat{\phi}_{1}-\phi_{0,1}\right)=-\left(D^{\prime} W D\right)^{-1} D^{\prime} W\left(\sqrt{T} m_{T}\left(\phi_{0}\right)+\frac{1}{2} G \sqrt{T}\left(\hat{\phi}_{p_{\phi}}-\phi_{0, p_{\phi}}\right)^{2}\right)+o_{P}(1) .
$$

In the direction of $\phi_{p_{\phi}}$, the first-order condition amounts to

$$
\begin{aligned}
& \left(G^{\prime} T^{1 / 4}\left(\hat{\phi}_{p_{\phi}}-\phi_{0, p_{\phi}}\right)+o_{P}(1)\right) \\
& \times \quad W\left(\sqrt{T} m_{T}\left(\phi_{0}\right)+D \sqrt{T}\left(\hat{\phi}_{1}-\phi_{0,1}\right)+\frac{1}{2} G \sqrt{T}\left(\hat{\phi}_{p_{\phi}}-\phi_{0, p_{\phi}}\right)^{2}+o_{P}(1)\right)=0 .
\end{aligned}
$$

The terms in the first parentheses are obtained by a first-order mean value expansion of $\frac{\partial m_{T}}{\partial \phi_{p_{\phi}}}(\hat{\phi})$ around $\phi_{0}$ and taking the limit. Plugging (49) into (50), we get:

$$
\begin{array}{ll} 
& T^{1 / 4}\left(\hat{\phi}_{p_{\phi}}-\phi_{0, p_{\phi}}\right) \\
\times \quad\left(G^{\prime} W^{1 / 2} M_{d} W^{1 / 2} \sqrt{T} m_{T}\left(\phi_{0}\right)+\frac{1}{2} G^{\prime} W^{1 / 2} M_{d} W^{1 / 2} G \sqrt{T}\left(\hat{\phi}_{p_{\phi}}-\phi_{0, p_{\phi}}\right)^{2}\right)=o_{P}(1) .
\end{array}
$$


Since $\sqrt{T} m_{T}\left(\phi_{0}\right)$ and $T^{1 / 4}\left(\hat{\phi}_{p_{\phi}}-\phi_{0, p_{\phi}}\right)$ are $O_{P}(1)$, the pair is jointly $O_{P}(1)$ and by the Prohorov's theorem, any subsequence of them has a further subsequence that jointly converges in distribution towards, say, $\left(\mathbb{Z}_{0}, \mathbb{V}_{0}\right)$. From $(51),\left(\mathbb{Z}_{0}, \mathbb{V}_{0}\right)$ satisfies:

$$
\mathbb{V}_{0}\left(\mathbb{Z}+\frac{1}{2} G^{\prime} W^{1 / 2} M_{d} W^{1 / 2} G \mathbb{V}_{0}^{2}\right)=0
$$

almost surely with $\mathbb{Z}=G^{\prime} W^{1 / 2} M_{d} W^{1 / 2} \mathbb{Z}_{0}$. Clearly, if $\mathbb{Z} \geq 0$, then, $\mathbb{V}_{0}=0$, almost surely. Conversely, following the proof of Dovonon and Renault (2013, Proposition 3.2), we can show that if $\mathbb{Z}<0$, then $\mathbb{V}_{0} \neq 0$, almost surely, and hence $\mathbb{V}_{0}^{2}=-2 \mathbb{Z} / G^{\prime} W^{1 / 2} M_{d} W^{1 / 2} G$.

In either case, $\mathbb{V}_{0}^{2}=-2 \frac{\mathbb{Z I}(\mathbb{Z}<0)}{G^{\prime} W^{1 / 2} M_{d} W^{1 / 2} G}(\equiv \mathbb{V})$ and is the limit distribution of the relevant subsequence of $\sqrt{T}\left(\hat{\phi}_{p_{\phi}}-\phi_{0, p_{\phi}}\right)^{2}$. Hence, that subsequence of $\left(\sqrt{T} m_{T}\left(\phi_{0}\right), \sqrt{T}\left(\hat{\phi}_{p_{\phi}}-\phi_{0, p_{\phi}}\right)^{2}\right)$ converges in distribution towards $\left(\mathbb{Z}_{0}, \mathbb{V}\right)$. The fact that this limit does not depend on a specific subsequence means that the whole sequence converges in distribution to that limit. We use (49) to conclude.

Next, we establish (c). We recall that the result in (b) gives the asymptotic distribution of $\sqrt{T}\left(\hat{\phi}_{p_{\phi}}-\right.$ $\left.\phi_{0, p_{\phi}}\right)^{2}$. To get the asymptotic distribution of $T^{1 / 4}\left(\hat{\phi}_{p_{\phi}}-\phi_{0, p_{\phi}}\right)$, it suffices to characterize its sign. Following the approach of Rotnitzky, Cox, Bottai, and Robins (2000) for MLE, we can do this by expanding $m_{T}^{\prime}(\hat{\phi}) W_{T} m_{T}(\hat{\phi})$ up to $o_{P}\left(T^{-5 / 4}\right)$. Being of order $O_{P}\left(T^{-1}\right)$, its $O_{P}\left(T^{-5 / 4}\right)$ terms actually provide the sign of $\left(\hat{\phi}_{p_{\phi}}-\phi_{0, p_{\phi}}\right)$; leading to the asymptotic distribution of $\left(\sqrt{T}\left(\hat{\phi}_{1}-\phi_{0,1}\right), T^{1 / 4}\left(\hat{\phi}_{p_{\phi}}-\phi_{0, p_{\phi}}\right)\right)$. By a mean value expansion of $m_{T}(\hat{\phi})$ up to the third order, we have:

$$
\begin{aligned}
& m_{T}(\hat{\phi}) \\
= & m_{T}\left(\phi_{0}\right)+\frac{\partial m_{T}}{\partial \phi_{1}^{\prime}}\left(\phi_{0}\right)\left(\hat{\phi}_{1}-\phi_{0,1}\right)+\frac{\partial m_{T}}{\partial \phi_{p}}\left(\phi_{0}\right)\left(\hat{\phi}_{p_{\phi}}-\phi_{0, p_{\phi}}\right)+\frac{1}{2} \frac{\partial^{2} m_{T}}{\partial \phi_{p_{\phi}}^{2}}\left(\phi_{0}\right)\left(\hat{\phi}_{p_{\phi}}-\phi_{0, p_{\phi}}\right)^{2} \\
& +\frac{\partial^{2} m_{T}}{\partial \phi_{p_{\phi}} \partial \phi_{1}^{\prime}}\left(\phi_{0}\right)\left(\hat{\phi}_{1}-\phi_{0,1}\right)\left(\hat{\phi}_{p_{\phi}}-\phi_{0, p_{\phi}}\right)+\frac{1}{6} \frac{\partial^{3} m_{T}}{\partial \phi_{p_{\phi}}^{3}}(\dot{\phi})\left(\hat{\phi}_{p_{\phi}}-\phi_{0, p_{\phi}}\right)^{3}+O_{P}\left(T^{-1}\right),
\end{aligned}
$$

where $\dot{\phi} \in\left(\phi_{0}, \hat{\phi}\right)$ and may differ from row to row. From Assumption 5(i), we obtain:

$$
\begin{aligned}
m_{T}(\hat{\phi})= & m_{T}\left(\phi_{0}\right)+D\left(\hat{\phi}_{1}-\phi_{0,1}\right)+\frac{\partial m_{T}}{\partial \phi_{p_{\phi}}}\left(\phi_{0}\right)\left(\hat{\phi}_{p_{\phi}}-\phi_{0, p_{\phi}}\right)+\frac{1}{2} G\left(\hat{\phi}_{p_{\phi}}-\phi_{0, p_{\phi}}\right)^{2} \\
& +G_{1 p_{\phi}}\left(\hat{\phi}_{1}-\phi_{0,1}\right)\left(\hat{\phi}_{p_{\phi}}-\phi_{0, p_{\phi}}\right)+\frac{1}{6} L\left(\hat{\phi}_{p_{\phi}}-\phi_{0, p_{\phi}}\right)^{3}+o_{P}\left(T^{-3 / 4}\right) .
\end{aligned}
$$

Hence, defining $Z_{0 T}=m_{T}\left(\phi_{0}\right)$ and $Z_{1 T}=\frac{\partial m_{T}}{\partial \phi_{p_{\phi}}}\left(\phi_{0}\right)$, it follows that:

$$
\begin{aligned}
m_{T}(\hat{\phi})= & Z_{0 T}+D\left(\hat{\phi}_{1}-\phi_{0,1}\right)+Z_{1 T}\left(\hat{\phi}_{p_{\phi}}-\phi_{0, p_{\phi}}\right)+\frac{1}{2} G\left(\hat{\phi}_{p_{\phi}}-\phi_{0, p_{\phi}}\right)^{2} \\
& +G_{1 p_{\phi}}\left(\hat{\phi}_{1} 1-\phi_{0,1}\right)\left(\hat{\phi}_{p_{\phi}}-\phi_{0, p_{\phi}}\right)+\frac{1}{6} L\left(\hat{\phi}_{p_{\phi}}-\phi_{0, p_{\phi}}\right)^{3}+o_{P}\left(T^{-3 / 4}\right) .
\end{aligned}
$$

The first-order condition for the $\hat{\phi}$ in the direction of $\phi_{1}$ is:

$$
0=\frac{\partial m_{T}^{\prime}}{\partial \phi_{1}}(\hat{\phi}) W_{T} m_{T}(\hat{\phi})=\left(D+G_{1 p_{\phi}}\left(\hat{\phi}_{p_{\phi}}-\phi_{0, p_{\phi}}\right)\right)^{\prime} W m_{T}(\hat{\phi})+o_{P}\left(T^{-3 / 4}\right)
$$

Substituting (52) into (53), we obtain an equation of the form:

$$
D^{\prime} W Z_{0 T}+D^{\prime} W D\left(\hat{\phi}_{1}-\phi_{0,1}\right)+\text { other terms }=o_{P}\left(T^{-3 / 4}\right) .
$$

This equation can be solved to yield:

$$
\hat{\phi}_{1}-\phi_{0,1}=-\left(D^{\prime} W D\right)^{-1}\left(D^{\prime} W Z_{0 T}+\text { other terms }\right)+o_{P}\left(T^{-3 / 4}\right) .
$$


Note that "other terms" contains $\hat{\phi}_{1}-\phi_{0,1}$ involved in quadratic functions of $\hat{\phi}-\phi_{0}$. Replacing in "other terms" $\hat{\phi}_{1}-\phi_{0,1}$ by this expression and keeping only the leading terms (up to $O_{P}\left(T^{-3 / 4}\right)$ ), we obtain the following expression for $\hat{\phi}_{1}-\phi_{0,1}$ :

$$
\begin{aligned}
\hat{\phi}_{1}-\phi_{0,1}= & H\left(Z_{0 T}+\left(Z_{1 T}+G_{1 p_{\phi}} H Z_{0 T}\right)\left(\hat{\phi}_{p_{\phi}}-\phi_{0, p_{\phi}}\right)+\frac{1}{2} G\left(\hat{\phi}_{p_{\phi}}-\phi_{0, p_{\phi}}\right)^{2}\right. \\
& \left.+\left(\frac{1}{2} G_{1 p_{\phi}} H G+\frac{1}{6} L\right)\left(\hat{\phi}_{p_{\phi}}-\phi_{0, p_{\phi}}\right)^{3}\right) \\
& +H_{1}\left(\left(Z_{0 T}+D H Z_{0 T}\right)\left(\hat{\phi}_{p_{\phi}}-\phi_{0, p_{\phi}}\right)+\frac{1}{2}(D H G+G)\left(\hat{\phi}_{p_{\phi}}-\phi_{0, p_{\phi}}\right)^{3}\right) \\
& +o_{P}\left(T^{-3 / 4}\right) \\
= & H\left(Z_{0 T}+\frac{1}{2} G\left(\hat{\phi}_{p_{\phi}}-\phi_{0, p_{\phi}}\right)^{2}\right) \\
& +\left(H Z_{1 T}+H G_{1 p_{\phi}} H Z_{0 T}+H_{1} Z_{0 T}+H_{1} D H Z_{0 T}\right)\left(\hat{\phi}_{p_{\phi}}-\phi_{0, p_{\phi}}\right) \\
& +\frac{1}{2}\left(H\left(G_{1 p_{\phi}} H G+\frac{L}{3}\right)+H_{1}(D H G+G)\right)\left(\hat{\phi}_{p_{\phi}}-\phi_{0, p_{\phi}}\right)^{3}+o_{P}\left(T^{-3 / 4}\right) .
\end{aligned}
$$

with $H=-\left(D^{\prime} W D\right)^{-1} D^{\prime} W$ and $H_{1}=-\left(D^{\prime} W D\right)^{-1} G_{1 p_{\phi}}^{\prime} W$. Hence, for a natural definition of $A_{1}, B_{1}$ and $C_{1},\left(\hat{\phi}^{1}-\phi_{0}^{1}\right)$ has the form:

$$
\left(\hat{\phi}_{1}-\phi_{0,1}\right)=A_{1}+B_{1}\left(\hat{\phi}_{p_{\phi}}-\phi_{0, p_{\phi}}\right)+C_{1}\left(\hat{\phi}_{p_{\phi}}-\phi_{0, p_{\phi}}\right)^{3}+o_{P}\left(T^{-3 / 4}\right)
$$

Using (52), we have:

$$
\begin{aligned}
& m_{T}^{\prime}(\hat{\phi}) W_{T} m_{T}(\hat{\phi})=m_{T}^{\prime}(\hat{\phi}) W m_{T}(\hat{\phi})+o_{P}\left(T^{-5 / 4}\right) \\
= & Z_{0 T}^{\prime} W Z_{0 T}+\left(\hat{\phi}_{1}-\phi_{0,1}\right)^{\prime} D^{\prime} W D\left(\hat{\phi}_{1}-\phi_{0,1}\right)+\frac{1}{4} G^{\prime} W G\left(\hat{\phi}_{p_{\phi}}-\phi_{0, p_{\phi}}\right)^{4} \\
& +2 Z_{0 T}^{\prime} W D\left(\hat{\phi}_{1}-\phi_{0,1}\right)+2 Z_{0 T}^{\prime} W Z_{1 T}\left(\hat{\phi}_{p_{\phi}}-\phi_{0, p_{\phi}}\right)+Z_{0 T}^{\prime} W G\left(\hat{\phi}_{p_{\phi}}-\phi_{0, p_{\phi}}\right)^{2} \\
& +2 Z_{0 T}^{\prime} W G_{1 p_{\phi}}\left(\hat{\phi}_{1}-\phi_{0,1}\right)\left(\hat{\phi}_{p_{\phi}}-\phi_{0, p_{\phi}}\right)+\frac{1}{3} Z_{0 T}^{\prime} W L\left(\hat{\phi}_{p_{\phi}}-\phi_{0, p_{\phi}}\right)^{3} \\
& +2\left(\hat{\phi}_{1}-\phi_{0,1}\right)^{\prime} D^{\prime} W Z_{1 T}\left(\hat{\phi}_{p_{\phi}}-\phi_{0, p_{\phi}}\right)+\left(\hat{\phi}_{1}-\phi_{0,1}\right)^{\prime} D^{\prime} W G\left(\hat{\phi}_{p_{\phi}}-\phi_{0, p_{\phi}}\right)^{2} \\
& +2\left(\hat{\phi}_{1}-\phi_{0,1}\right)^{\prime} D^{\prime} W G_{1 p_{\phi}}\left(\hat{\phi}_{1}-\phi_{0,1}\right)\left(\hat{\phi}_{p_{\phi}}-\phi_{0, p_{\phi}}\right)+\frac{1}{3}\left(\hat{\phi}_{1}-\phi_{0,1}\right)^{\prime} D^{\prime} W L\left(\hat{\phi}_{p_{\phi}}-\phi_{0, p_{\phi}}\right)^{3} \\
& +Z_{1 T}^{\prime} W G\left(\hat{\phi}_{p_{\phi}}-\phi_{0, p_{\phi}}\right)^{3}+G^{\prime} W G_{1 p_{\phi}}\left(\hat{\phi}_{1}-\phi_{0,1}\right)\left(\hat{\phi}_{p_{\phi}}-\phi_{0, p_{\phi}}\right)^{3} \\
& +\frac{1}{6} G^{\prime} W L\left(\hat{\phi}_{p_{\phi}}-\phi_{0, p_{\phi}}\right)^{5}+o_{P}\left(T^{-5 / 4}\right) .
\end{aligned}
$$

Replacing $\hat{\phi}_{1}-\phi_{0,1}$ by its expression from (56) into (57), the leading $O_{P}\left(T^{-1}\right)$ term of $m_{T}^{\prime}(\hat{\phi}) W_{T} m_{T}(\hat{\phi})$ is obtained as $K_{T}\left(\hat{\phi}_{p_{\phi}}\right)$ with

$$
\begin{aligned}
& K_{T}\left(\phi_{p_{\phi}}\right) \\
= & Z_{0 T}^{\prime} W Z_{0 T}+\left(Z_{0 T}+\frac{1}{2} G\left(\phi_{p_{\phi}}-\phi_{0 p}\right)^{2}\right)^{\prime} H^{\prime} D^{\prime} W D H\left(Z_{0 T}+\frac{1}{2} G\left(\phi_{p_{\phi}}-\phi_{0, p_{\phi}}\right)^{2}\right) \\
& +\frac{1}{4} G^{\prime} W G\left(\phi_{p_{\phi}}-\phi_{0, p_{\phi}}\right)^{4}+2 Z_{0 T}^{\prime} W D H\left(Z_{0 T}+\frac{1}{2} G\left(\phi_{p_{\phi}}-\phi_{0, p_{\phi}}\right)^{2}\right) \\
& +Z_{0 T}^{\prime} W G\left(\phi_{p_{\phi}}-\phi_{0, p_{\phi}}\right)^{2}+\left(Z_{0 T}+\frac{1}{2} G\left(\phi_{p_{\phi}}-\phi_{0, p_{\phi}}\right)^{2}\right)^{\prime} H^{\prime} D^{\prime} W G\left(\phi_{p_{\phi}}-\phi_{0, p_{\phi}}\right)^{2} .
\end{aligned}
$$


Hence,

$$
\begin{aligned}
K_{T}\left(\phi_{p_{\phi}}\right)= & Z_{0 T}^{\prime} W^{1 / 2} M_{d} W^{1 / 2} Z_{0 T}+Z_{0 T}^{\prime} W^{1 / 2} M_{d} W^{1 / 2} G\left(\phi_{p_{\phi}}-\phi_{0, p_{\phi}}\right)^{2} \\
& +\frac{1}{4} G^{\prime} W^{1 / 2} M_{d} W^{1 / 2} G\left(\phi_{p_{\phi}}-\phi_{0, p_{\phi}}\right)^{4} .
\end{aligned}
$$

The next leading term in the expansion of $m_{T}^{\prime}(\hat{\phi}) W_{T} m_{T}(\hat{\phi})$ is of order $O_{P}\left(T^{-5 / 4}\right)$ and given by:

$$
\begin{aligned}
& R_{T}=\left(\hat{\phi}_{p_{\phi}}-\phi_{0, p_{\phi}}\right) \times \\
& \left\{2 A_{1}^{\prime} D^{\prime} W D B_{1}+2 Z_{0 T}^{\prime} W D B_{1}+2 Z_{0 T}^{\prime} W Z_{1 T}\right. \\
& +2 Z_{0 T}^{\prime} W G_{1 p_{\phi}} A_{1}+2 A_{1}^{\prime} D^{\prime} W Z_{1 T}+2 A_{1}^{\prime} D^{\prime} W G_{1 p_{\phi}} A_{1} \\
& +\left(\hat{\phi}_{p_{\phi}}-\phi_{0, p_{\phi}}\right)^{2}\left(2 A_{1}^{\prime} D^{\prime} W D C_{1}+2 Z_{0 T}^{\prime} W D C_{1}+\frac{1}{3} Z_{0 T}^{\prime} W L+B_{1}^{\prime} D^{\prime} W G\right. \\
& \left.+\frac{1}{3} A_{1}^{\prime} D^{\prime} W L+Z_{1 T}^{\prime} W G+G^{\prime} W G_{1 p_{\phi}} A_{1}\right) \\
& \left.+\left(\hat{\phi}_{p_{\phi}}-\phi_{0, p_{\phi}}\right)^{4}\left(C_{1}^{\prime} D^{\prime} W G+\frac{1}{6} G^{\prime} W L\right)\right\} \\
& R_{T}=\left(\hat{\phi}_{p_{\phi}}-\phi_{0, p_{\phi}}\right) \times \\
& \left\{2 Z_{0 T}^{\prime} H^{\prime} D^{\prime} W D B_{1}+2 Z_{0 T}^{\prime} W D B_{1}+2 Z_{0 T}^{\prime} W Z_{1 T}+2 Z_{0 T}^{\prime} W G_{1 p_{\phi}} H Z_{0 T}\right. \\
& +2 Z_{0 T}^{\prime} H^{\prime} D^{\prime} W Z_{1 T}+2 Z_{0 T}^{\prime} H^{\prime} D^{\prime} W G_{1 p_{\phi}} H Z_{0 T} \\
& +\left(\hat{\phi}_{p_{\phi}}-\phi_{0, p_{\phi}}\right)^{2} \quad\left(2 Z_{0 T}^{\prime} H^{\prime} D^{\prime} W D C_{1}+2 Z_{0 T}^{\prime} W D C_{1}+\frac{1}{3} Z_{0 T}^{\prime} W L+B_{1}^{\prime} D^{\prime} W G\right. \\
& +\frac{1}{3} Z_{0 T}^{\prime} H^{\prime} D^{\prime} W L+Z_{1 T}^{\prime} W G+G^{\prime} W G_{1 p_{\phi}} H Z_{0 T}+G^{\prime} H^{\prime} D^{\prime} W D B_{1} \\
& \left.+G^{\prime} H^{\prime} D^{\prime} W Z_{1 T}+Z_{0 T}^{\prime} W G_{1 p_{\phi}} H G+Z_{0 T}^{\prime} H^{\prime} D^{\prime} W G_{1 p_{\phi}} H G+G^{\prime} H^{\prime} D^{\prime} W G_{1 p_{\phi}} H Z_{0 T}\right) \\
& +\left(\hat{\phi}_{p_{\phi}}-\phi_{0, p_{\phi}}\right)^{4} \quad\left(C_{1}^{\prime} D^{\prime} W G+\frac{1}{6} G^{\prime} W L+\frac{1}{2} G^{\prime} H^{\prime} D^{\prime} W G_{1 p_{\phi}} H G+G^{\prime} H^{\prime} D^{\prime} W D C_{1}\right. \\
& \left.\left.+\frac{1}{6} G^{\prime} H^{\prime} D^{\prime} W L+\frac{1}{2} G^{\prime} W G_{1 p_{\phi}} H G\right)\right\} \equiv\left(\hat{\phi}_{p_{\phi}}-\phi_{0, p_{\phi}}\right) \times 2 R_{1 T} .
\end{aligned}
$$

Re-arranging the terms and using the fact that $M_{d} W^{1 / 2} D=0$, we have:

$$
\begin{aligned}
2 R_{1 T}= & 2 Z_{0 T}^{\prime} W^{1 / 2} M_{d} W^{1 / 2} Z_{1 T}+2 Z_{0 T}^{\prime} W^{1 / 2} M_{d} W^{1 / 2} G_{1 p_{\phi}} H Z_{0 T} \\
& +\left(\hat{\phi}_{p_{\phi}}-\phi_{0, p_{\phi}}\right)^{2}\left(\frac{1}{3} Z_{0 T}^{\prime} W^{1 / 2} M_{d} W^{1 / 2} L+Z_{1 T}^{\prime} W^{1 / 2} M_{d} W^{1 / 2} G\right. \\
& \left.+G^{\prime} W^{1 / 2} M_{d} W^{1 / 2} G_{1 p_{\phi}} H Z_{0 T}+Z_{0 T}^{\prime} W^{1 / 2} M_{d} W^{1 / 2} G_{1 p_{\phi}} H G\right) \\
& +\left(\hat{\phi}_{p_{\phi}}-\phi_{0, p_{\phi}}\right)^{4}\left(\frac{1}{6} G^{\prime} W^{1 / 2} M_{d} W^{1 / 2} L+\frac{1}{2} G^{\prime} W^{1 / 2} M_{d} W^{1 / 2} G_{1 p_{\phi}} H G\right) .
\end{aligned}
$$

We can check that the GMM estimator $\hat{\phi}_{p_{\phi}}$ as given by the first-order condition (51) is minimizer of 
$K_{T}\left(\phi_{p_{\phi}}\right)$. When $T^{1 / 4}\left(\hat{\phi}_{p_{\phi}}-\phi_{0, p_{\phi}}\right)$ is not $o_{P}(1)$, this first-order condition determines

$$
\left(\hat{\phi}_{p_{\phi}}-\phi_{0, p_{\phi}}\right)^{2}=-2 \frac{G^{\prime} W^{1 / 2} M_{d} W^{1 / 2} Z_{0 T}}{G^{\prime} W^{1 / 2} M_{d} W^{1 / 2} G}+o_{P}\left(T^{-1 / 2}\right)
$$

but not the sign of $\left(\hat{\phi}_{p_{\phi}}-\phi_{0, p_{\phi}}\right)$. Following the analysis of Rotnitzky, Cox, Bottai, and Robins (2000) for the maximum likelihood estimator, the sign of $\hat{\phi}_{p_{\phi}}-\phi_{0, p_{\phi}}$ can be determined by the remainder $R_{T}$ of the expansion of $m_{T}^{\prime}(\hat{\phi}) W_{T} m_{T}(\hat{\phi})$. At the minimum, we expect $R_{T}$ to be negative; i.e. $\left(\hat{\phi}_{p_{\phi}}-\phi_{0, p_{\phi}}\right)$ and $R_{1 T}$ have opposite sign.

Hence,

$$
T^{1 / 4}\left(\hat{\phi}_{p_{\phi}}-\phi_{0, p_{\phi}}\right)=(-1)^{B_{T}} T^{1 / 4}\left|\hat{\phi}_{p_{\phi}}-\phi_{0, p_{\phi}}\right|
$$

with $B_{T}=\mathbb{I}\left(T R_{1 T} \geq 0\right)$.

Plugging the expression of $\left(\hat{\phi}_{p_{\phi}}-\phi_{0, p_{\phi}}\right)^{2}$ into (59) and scaling by $T$, we can see, using the continuous mapping theorem, that $T R_{1 T}$ converges in distribution towards $\mathbb{R}_{1}$ :

$$
\begin{aligned}
\mathbb{R}_{1}= & \mathbb{Z}_{0}^{\prime} W^{1 / 2} M_{d g} W^{1 / 2}\left(\mathbb{Z}_{1}+G_{1 p_{\phi}} H \mathbb{Z}_{0}\right) \\
& +\left(\mathbb{Z}_{0}^{\prime} W^{1 / 2}\left(M_{d}-M_{d g}\right) W^{1 / 2} \mathbb{Z}_{0} G^{\prime}-G^{\prime} W^{1 / 2} M_{d} W^{1 / 2} \mathbb{Z}_{0} \mathbb{Z}_{0}^{\prime}\right) \\
& \times W^{1 / 2} M_{d} W^{1 / 2}\left(\frac{1}{3} L+G_{1 p_{\phi}} H G\right) / \sigma_{G},
\end{aligned}
$$

with $\sigma_{G}=G^{\prime} W^{1 / 2} M_{d} W^{1 / 2} G$ and

$M_{d g}=M_{d}-M_{d} W^{1 / 2} G\left(G^{\prime} W^{1 / 2} M_{d} W^{1 / 2} G\right)^{-1} G^{\prime} W^{1 / 2} M_{d}$, the matrix of the orthogonal projection on the orthogonal of $\left(\begin{array}{ll}W^{1 / 2} D & W^{1 / 2} G\end{array}\right)$.

We actually have that: $\left(\sqrt{T} Z_{0 T}, \sqrt{T} Z_{1 T}, T R_{1 T}\right)$ converges in distribution towards $\left(\mathbb{Z}_{0}, \mathbb{Z}_{1}, \mathbb{R}_{1}\right)$. Applying Lemma 1 , we have $\left(\sqrt{T} Z_{0 T}, \sqrt{T} Z_{1 T},(-1)^{B_{T}}\right) \stackrel{d}{\rightarrow}\left(\mathbb{Z}_{0}, \mathbb{Z}_{1},(-1)^{\mathbb{B}}\right)$, where $\mathbb{B}=\mathbb{I}\left(\mathbb{R}_{1} \geq 0\right)$.

Since $\left(\sqrt{T}\left(\hat{\phi}_{1}-\phi_{0,1}\right), T^{1 / 4}\left|\hat{\phi}_{p_{\phi}}-\phi_{0, p_{\phi}}\right|,(-1)^{B_{T}}\right)=O_{P}(1)$, any subsequence of the left hand side has a further subsequence that converges in distribution. Using (b), such subsequence satisfies:

$$
\left(\sqrt{T}\left(\hat{\phi}_{1}-\phi_{0,1}\right), T^{1 / 4}\left|\hat{\phi}_{p_{\phi}}-\phi_{0, p_{\phi}}\right|, \quad(-1)^{B_{T}}\right) \stackrel{d}{\rightarrow}\left(H \mathbb{Z}_{0}+H G \mathbb{V} / 2, \sqrt{\mathbb{V}},(-1)^{\mathbb{B}}\right) .
$$

(We keep $T$ to index the subsequence for simplicity.)

Since the limit distribution does not depend on the subsequence, the whole sequence converges towards that limit. By the continuous mapping theorem, we deduce that:

$$
\left(\sqrt{T}\left(\hat{\phi}_{1}-\phi_{0,1}\right), \quad T^{1 / 4}\left(\hat{\phi}_{p_{\phi}}-\phi_{0, p_{\phi}}\right)\right) \stackrel{d}{\rightarrow}\left(H \mathbb{Z}_{0}+H G \mathbb{V} / 2,(-1)^{\mathbb{B}} \sqrt{\mathbb{V}}\right) .
$$

Lemma 1. Let $\left(X_{T}\right)_{T}$ and $\left(Y_{T}\right)_{T}$ be two sequences of random variables and $B_{T}=\mathbb{I}\left(X_{T} \geq 0\right)$. If $\left(X_{T}, Y_{T}\right) \stackrel{d}{\rightarrow}(X, Y)$ and $P(X=0)=0$, then

$$
\left((-1)^{B_{T}}, Y_{T}\right) \stackrel{d}{\rightarrow}\left((-1)^{B}, Y\right),
$$

with $B=\mathbb{I}(X \geq 0)$. 
Proof of Lemma 1: Using the Cramer-Wold device, it suffices to show that: for all $\left(\lambda_{1}, \lambda_{2}\right) \in \mathbb{R} \times \mathbb{R}$,

$$
\lambda_{1}(-1)^{B_{T}}+\lambda_{2} Y_{T} \stackrel{d}{\rightarrow} \lambda_{1}(-1)^{B}+\lambda_{2} Y .
$$

Let $x \in \mathbb{R}$ be a continuity point of $F(x)=P\left(\lambda_{1}(-1)^{B}+\lambda_{2} Y \leq x\right)$. We show that:

$$
P\left(\lambda_{1}(-1)^{B_{T}}+\lambda_{2} Y_{T} \leq x\right) \rightarrow F(x), \quad \text { as } \quad T \rightarrow \infty .
$$

We have:

$$
P\left(\lambda_{1}(-1)^{B_{T}}+\lambda_{2} Y_{T} \leq x\right)=P\left(\lambda_{2} Y_{T} \leq x-\lambda_{1}, X_{T}<0\right)+P\left(\lambda_{2} Y_{T} \leq x+\lambda_{1}, X_{T} \geq 0\right) .
$$

To complete the proof, it suffices to show that, as $T \rightarrow \infty$,

$$
\begin{gathered}
P\left(\lambda_{2} Y_{T} \leq x-\lambda_{1}, X_{T}<0\right) \rightarrow P\left(\lambda_{2} Y \leq x-\lambda_{1}, X<0\right) \quad \text { and } \\
P\left(\lambda_{2} Y_{T} \leq x+\lambda_{1}, X_{T} \geq 0\right) \rightarrow P\left(\lambda_{2} Y \leq x+\lambda_{1}, X \geq 0\right)
\end{gathered}
$$

since $F(x)=P\left(\lambda_{2} Y \leq x-\lambda_{1}, X<0\right)+P\left(\lambda_{2} Y \leq x+\lambda_{1}, X \geq 0\right)$.

We now establish the first condition in (61). The second one is obtained along the same lines. Note that $P\left(\lambda_{2} Y_{T} \leq x-\lambda_{1}, X_{T}<0\right)=P\left(\left(\lambda_{2} Y_{T}, X_{T}\right) \in A\right)$ with boundary of $A$ given by: $\partial A=$ $\left(\left(-\infty, x-\lambda_{1}\right] \times\{0\}\right) \bigcup\left(\left\{x-\lambda_{1}\right\} \times(-\infty, 0]\right)$. Since $\left(X_{T}, Y_{T}\right)$ converge jointly in distribution towards $(X, Y)$, it suffices to show that

$$
P\left(\left(\lambda_{2} Y, X\right) \in \partial A\right)=0 .
$$

We have:

$$
P\left(\left(\lambda_{2} Y, X\right) \in\left(-\infty, x-\lambda_{1}\right] \times\{0\}\right) \leq P(X=0)=0 .
$$

Besides,

$$
P\left(\left(\lambda_{2} Y, X\right) \in\left\{x-\lambda_{1}\right\} \times(-\infty, 0]\right)=P\left(\lambda_{2} Y=x-\lambda_{1}, X \leq 0\right) .
$$

By continuity of $F$ at $x, P\left(\lambda_{1}(-1)^{B}+\lambda_{2} Y=x\right)=0$, i.e.

$$
P\left(\lambda_{2} Y=x+\lambda_{1}, X \geq 0\right)+P\left(\lambda_{2} Y=x-\lambda_{1}, X<0\right)=0 .
$$

Thus, $P\left(\lambda_{2} Y=x-\lambda_{1}, X<0\right)=0$. Since $P(X=0)=0$, we can claim that

$$
P\left(\lambda_{2} Y=x+\lambda_{1}, X \leq 0\right)=0 .
$$

This completes the proof.

Proof of Equation (19): First, we observe that the asymptotic distribution $\mathbb{V}$ of $T^{1 / 2}\left(\hat{\phi}-\phi_{0}\right)^{2}$ is continuous at any $c>0$ (with $P(\mathbb{V}=0)=1 / 2$ ). Let us search for $c_{1-\alpha}$ such that $P\left(\mathbb{V} \leq c_{1-\alpha}\right)=1-\alpha$. Since $1-\alpha>1 / 2$, we have $c_{1-\alpha}>0$ and $P\left(T^{1 / 4}\left|\hat{\phi}-\phi_{0}\right| \leq \sqrt{c_{1-\alpha}}\right) \rightarrow 1-\alpha$, as $T \rightarrow \infty$. Hence, $\sqrt{c_{1-\alpha}}$ defines an asymptotically correct confidence interval for $\phi_{0}$. To obtain $c_{1-\alpha}$, we recall that:

$$
\begin{aligned}
& P\left(\mathbb{V} \leq c_{1-\alpha}\right)=P\left(-\frac{2 \mathbb{Z} \mathbb{(}(<0)}{\sigma_{G}} \leq c_{1-\alpha}\right) \\
= & P\left(-\frac{2 \mathbb{Z I}(\mathbb{Z}<0)}{\sigma_{G}} \leq c_{1-\alpha}, \mathbb{Z} \geq 0\right)+P\left(-\frac{2 \mathbb{Z I}(\mathbb{Z}<0)}{\sigma_{G}} \leq c_{1-\alpha}, \mathbb{Z}<0\right)=P\left(\mathbb{Z} \leq \frac{\sigma_{G}}{2} c_{1-\alpha}\right) .
\end{aligned}
$$

The last equality uses the fact that $\mathbb{Z}$ has a symmetric distribution about 0 as a zero mean Gaussian variable. Since $Z \sim N\left(0, G^{\prime} W \Omega W G\right), c_{1-\alpha}$ solves:

$$
\frac{\sigma_{G}}{2 \sqrt{G^{\prime} W \Omega W G}} c_{1-\alpha}=z_{\alpha}
$$


giving $c_{1-\alpha}=\frac{2 \sqrt{G^{\prime} W \Omega W G}}{\sigma_{G}} z_{\alpha}$.

When a consistent estimator $\hat{c}_{1-\alpha}$ of $c_{1-\alpha}$ is used as in the statement of (19), we can rely on the following two facts to conclude: (1) $\mathbb{V}$ has a continuous distribution at all $c>0$ and (2) if $X_{n}$ is a sequence of random variables converging in distribution to $X$ with cumulative distribution function $F_{X}$ that is continuous on an interval $[a, b]$, then the sequence of cumulative distributions $F_{X_{n}}$ of $X_{n}$ converges uniformly over $[a, b]$ to $F_{X}$.

Proof of Equation (23): Since $\hat{\phi}-\phi_{0}=R\left(\hat{\eta}-\eta_{0}\right)$, we have

$$
T^{1 / 4}\left(\hat{\phi}-\phi_{0}\right)=R_{\bullet p_{\phi}} T^{1 / 4}\left(\hat{\eta}_{p_{\phi}}-\eta_{0, p_{\phi}}\right) .
$$

We also have

$$
\epsilon_{T}=-B_{T} \hat{R}^{-1}(\hat{R}-R) R^{-1}\left(\hat{\phi}-\phi_{0}\right)=-B_{T} \hat{R}^{-1}(\hat{R}-R)\left(\hat{\eta}_{p_{\phi}}-\eta_{0, p_{\phi}}\right) .
$$

But $\hat{R} \equiv R(\hat{\phi})$ and $R \equiv R\left(\phi_{0}\right)$. By mean value expansions, for $j=1, \ldots, p_{\phi}$,

$$
\hat{R}_{\bullet j}-R_{\bullet j}=\frac{\partial R_{\bullet} j}{\partial \phi^{\prime}}\left(\dot{\phi}_{j}\right)\left(\hat{\phi}-\phi_{0}\right),
$$

where $\dot{\phi}_{j} \in\left(\phi_{0}, \hat{\phi}\right)$ and may differ from row to row and $R_{\bullet j}$ denotes the column vector corresponding to the $j$ th column of the matrix $R$. We also use $R_{h}$ to denote the row vector corresponding to the $h$ th row of $R$.

For $h=1, \ldots, p_{\phi}-1$,

$$
\begin{aligned}
\epsilon_{T, h} & =-\sqrt{T}\left(\hat{R}^{-1}\right)_{h \bullet} \sum_{j=1}^{p_{\phi}}\left(\frac{\partial R_{\bullet j}}{\partial \phi^{\prime}}\left(\dot{\phi}_{j}\right)\left(\hat{\phi}-\phi_{0}\right)\right)\left(\hat{\eta}_{j}-\eta_{0, j}\right) \\
& =-\left(\hat{R}^{-1}\right)_{h \bullet} \sum_{j=1}^{p_{\phi}}\left(\frac{\partial R_{\bullet} j}{\partial \phi^{\prime}}\left(\dot{\phi}_{j}\right) T^{1 / 4}\left(\hat{\phi}-\phi_{0}\right)\right) T^{1 / 4}\left(\hat{\eta}_{j}-\eta_{0, j}\right) \\
& =-\left(R^{-1}\right)_{h \bullet} \frac{\partial R \bullet p_{\phi}}{\partial \phi^{\prime}}\left(\phi_{0}\right) R \bullet_{p_{\phi}}\left(T^{1 / 4}\left(\hat{\eta}_{p_{\phi}}-\eta_{0, p_{\phi}}\right)\right)^{2}+o_{P}(1),
\end{aligned}
$$

where the last equality uses $(62)$ and the fact that $\hat{R}$ and $\frac{\partial R_{\bullet} j}{\partial \phi^{\prime}}\left(\dot{\phi}_{j}\right)$ converge in probability towards $R$ and $\frac{\partial R_{\bullet} j}{\partial \phi^{\prime}}\left(\phi_{0}\right)$, respectively and the fact that $T^{1 / 4}\left(\hat{\eta}_{j}-\eta_{0, j}\right)=o_{P}(1)$ for $j=1, \ldots, p_{\phi}-1$.

Besides, we have

$$
\epsilon_{T, p_{\phi}}=-\left(\hat{R}^{-1}\right)_{p_{\phi} \bullet} \sum_{j=1}^{p_{\phi}}\left(\frac{\partial R_{\bullet j}}{\partial \phi^{\prime}}\left(\dot{\phi}_{j}\right) T^{1 / 4}\left(\hat{\phi}-\phi_{0}\right)\right)\left(\hat{\eta}_{j}-\eta_{0, j}\right)=o_{P}(1) .
$$

Putting together these last two equalities, we get:

$$
\epsilon_{T}=-A\left(T^{1 / 4}\left(\hat{\eta}_{p_{\phi}}-\eta_{0, p_{\phi}}\right)\right)^{2}+o_{P}(1)
$$

as expected.

Proof of Equation (27): A second-order mean value expansion yields:

$$
f\left(\phi_{0}\right)=f(\hat{\phi})+\frac{\partial f}{\partial \phi^{\prime}}(\hat{\phi})\left(\phi_{0}-\hat{\phi}\right)+\frac{1}{2}\left(\left(\phi_{0}-\hat{\phi}\right)^{\prime} \frac{\partial^{2} f_{k}}{\partial \phi \partial \phi^{\prime}}(\dot{\phi})\left(\phi_{0}-\hat{\phi}\right)\right)_{1 \leq k \leq K},
$$

with $\dot{\phi} \in\left(\phi_{0}, \hat{\phi}\right)$ and may vary from row to row. This can be re-written:

$$
f(\hat{\phi})-f\left(\phi_{0}\right)=\frac{\partial f}{\partial \phi^{\prime}}(\hat{\phi})\left(\hat{\phi}-\phi_{0}\right)-\frac{1}{2}\left(\left(\hat{\phi}-\phi_{0}\right)^{\prime} \frac{\partial^{2} f_{k}}{\partial \phi \partial \phi^{\prime}}(\dot{\phi})\left(\hat{\phi}-\phi_{0}\right)\right)_{1 \leq k \leq K} .
$$


From (26), we can claim that:

$$
f(\hat{\phi})-f\left(\phi_{0}\right) \stackrel{a}{\sim} \frac{\partial f}{\partial \phi^{\prime}}(\hat{\phi}) \hat{R} B_{T}^{-1} \tilde{\mathbb{X}}-\frac{1}{2}\left(\tilde{\mathbb{X}}^{\prime} B_{T}^{-1} \hat{R}^{\prime} \frac{\partial^{2} f_{k}}{\partial \phi \partial \phi^{\prime}}(\dot{\phi}) \hat{R} B_{T}^{-1} \tilde{\mathbb{X}}\right)_{1 \leq k \leq K} .
$$

Note that the first term on the RHS is of order $O_{P}\left(T^{-1 / 4}\right)$ or $O_{P}\left(T^{-1 / 2}\right)$ and cannot vanish trivially at a rate faster than $O_{P}\left(T^{-1 / 2}\right)$ if $\frac{\partial f}{\partial \phi^{\prime}}\left(\phi_{0}\right) \neq 0$ because $R$ and $\operatorname{Var}(\tilde{\mathbb{X}})$ are non singular. The only element from the expansion of the second term that does not vanish at a rate faster than $O_{P}\left(T^{-1 / 2}\right)$ is, with $\hat{R} \bullet p_{\phi}$ denoting the $p_{\phi}^{t h}$ column of $\hat{R}$,

$$
-\frac{1}{2} \frac{1}{\sqrt{T}}\left(\hat{R}_{\bullet p_{\phi}}^{\prime} \frac{\partial^{2} f_{k}}{\partial \phi \partial \phi^{\prime}}(\dot{\phi}) \hat{R} \bullet p_{\phi} \tilde{\mathbb{X}}_{p_{\phi}}^{2}\right)_{1 \leq k \leq K}
$$

Thus, we have

$$
f(\hat{\phi})-f\left(\phi_{0}\right) \stackrel{a}{\sim} \frac{\partial f}{\partial \phi^{\prime}}(\hat{\phi}) \hat{R} B_{T}^{-1} \tilde{\mathbb{X}}-\frac{1}{2} \frac{1}{\sqrt{T}}\left(\hat{R}_{\bullet p_{\phi}}^{\prime} \frac{\partial^{2} f_{k}}{\partial \phi \partial \phi^{\prime}}(\dot{\phi}) \hat{R}_{\bullet p_{\phi}} \tilde{\mathbb{X}}_{p_{\phi}}^{2}\right)_{1 \leq k \leq K} .
$$

Under standard conditions, we have:

$$
\frac{\partial^{2} f_{k}}{\partial \phi \partial \phi^{\prime}}(\dot{\phi})-\frac{\partial^{2} f_{k}}{\partial \phi \partial \phi^{\prime}}(\hat{\phi})=o_{P}(1)
$$

and using this result in (63) yields (27).

Proof of Theorem 2: We have:

$$
B_{I T} \hat{S}\left(\hat{\theta}_{I I}-\theta_{0}\right)=\left(\begin{array}{c}
\sqrt{T} \hat{S}_{1}\left(\hat{\theta}_{I I}-\theta_{0}\right) \\
T^{1 / 4} \hat{S}_{p \bullet}\left(\hat{\theta}_{11}-\theta_{0}\right)
\end{array}\right)
$$

where $\hat{S}_{p}$ is the $p^{\text {th }}$ row of $\hat{S}$. From (34), we have

$$
\begin{gathered}
\sqrt{T} \hat{S}_{1}\left(\hat{\theta}_{I I}-\theta_{0}\right)=\hat{S}_{1} \hat{F}_{T}\left(B_{T} m_{I T}\left(\theta_{0}\right)-\frac{1}{2} z_{T}\right), \\
\text { with } z_{T}=B_{T}\left(\left(\hat{\theta}_{I I}-\theta_{0}\right)^{\prime} \Delta_{I T, k}\left(\dot{\theta}_{T}\right)\left(\hat{\theta}_{I I}-\theta_{0}\right)\right)_{1 \leq k \leq \ell} \text {. For } k=1, \ldots, \ell-1, \\
z_{T, k}=\sqrt{T}\left(\hat{\theta}_{I I}-\theta_{0}\right)^{\prime} \Delta_{I T, k}\left(\dot{\theta}_{T}\right)\left(\hat{\theta}_{I I}-\theta_{0}\right)=T^{1 / 4}\left(\hat{\theta}_{I I}-\theta_{0}\right)^{\prime} \Delta_{I T, k}\left(\dot{\theta}_{T}\right) T^{1 / 4}\left(\hat{\theta}_{I I}-\theta_{0}\right)
\end{gathered}
$$

and

$$
z_{T, \ell}=T^{1 / 4}\left(\hat{\theta}_{I I}-\theta_{0}\right)^{\prime} \Delta_{I T, \ell}\left(\dot{\theta}_{T}\right)\left(\hat{\theta}_{I I}-\theta_{0}\right) .
$$

From (32), we have $T^{1 / 4}\left(\hat{\theta}_{I I}-\theta_{0}\right)=F_{\bullet} T^{1 / 4} m_{I T, \ell}\left(\theta_{0}\right)+o_{P}(1)$. In addition, the fact that $\Delta_{I T, k}\left(\dot{\theta}_{T}\right)$ converges in probability towards $\Delta_{I, k}\left(\theta_{0}\right)$ for all $k=1, \ldots, \ell$, allows us to claim that: for $1 \leq k \leq \ell-1$,

$$
z_{T, k}=F_{\bullet \ell}^{\prime} \Delta_{I, k}\left(\theta_{0}\right) F_{\bullet \ell}\left(T^{1 / 4} m_{I T, \ell}\left(\theta_{0}\right)\right)^{2}+o_{P}(1)
$$

and

$$
z_{T, \ell}=O_{P}(1) O_{P}(1) o_{P}(1)=o_{P}(1) .
$$

Thus,

$$
\begin{aligned}
& \sqrt{T} \hat{S}_{1}\left(\hat{\theta}_{I I}-\theta_{0}\right) \\
= & \hat{S}_{1} \hat{F}_{T}\left(B_{T} m_{I T}\left(\theta_{0}\right)-\frac{1}{2}\left(\begin{array}{c}
\left(F_{\bullet \ell}^{\prime} \Delta_{I, k}\left(\theta_{0}\right) F_{\bullet \ell}\right)_{1 \leq k \leq \ell-1} \\
0
\end{array}\right)\left(T^{1 / 4} m_{I T, \ell}\left(\theta_{0}\right)\right)^{2}\right)+o_{P}(1) .
\end{aligned}
$$


Since the last column of $\hat{S}_{1} \hat{F}_{T}$ is nil, we can write:

$$
\begin{aligned}
& \sqrt{T} \hat{S}_{1}\left(\hat{\theta}_{I I}-\theta_{0}\right) \\
= & \hat{S}_{1} \hat{F}_{T}\left(B_{T} m_{I T}\left(\theta_{0}\right)-\frac{1}{2}\left(F_{\bullet \ell}^{\prime} \Delta_{I, k}\left(\theta_{0}\right) F_{\bullet \ell}\right)_{1 \leq k \leq \ell}\left(T^{1 / 4} m_{I T, \ell}\left(\theta_{0}\right)\right)^{2}\right)+o_{P}(1) .
\end{aligned}
$$

Using again (32), we have

$$
T^{1 / 4} \hat{S}_{p \bullet}\left(\hat{\theta}_{I I}-\theta_{0}\right)=\hat{S}_{p \bullet} F_{\bullet} T^{1 / 4} m_{I T, \ell}\left(\theta_{0}\right)+o_{P}(1) .
$$

By the continuous mapping theorem, $\hat{S}_{1} \hat{F}_{T}$ converges in probability towards $S_{1} F$ with nil last column and $\hat{S}_{p} \bullet$ converges in probability towards $S_{p}$. Since $B_{T} m_{I T}\left(\theta_{0}\right)$ converges in distribution towards $\mathbb{Y}$, we can deduce from (64) and (65) that:

$$
\left(\begin{array}{c}
\sqrt{T} \hat{S}_{1}\left(\hat{\theta}_{I I}-\theta_{0}\right) \\
T^{1 / 4} \hat{S}_{p \bullet}\left(\hat{\theta}_{11}-\theta_{0}\right)
\end{array}\right) \stackrel{d}{\rightarrow}\left(\begin{array}{c}
S_{1} F\left(\mathbb{Y}-\frac{\left(\mathbb{Y}_{\ell}\right)^{2}}{2}\left(F_{\bullet \ell}^{\prime} \Delta_{I, k}\left(\theta_{0}\right) F_{\bullet \ell}\right)_{1 \leq k \leq \ell}\right) \\
S_{p \bullet} F_{\bullet \ell} \mathbb{Y}_{\ell}
\end{array}\right)
$$




\section{Tables and graphs}

Table 1: GMM estimation of panel data model

\begin{tabular}{|c|c|c|c|c|c|c|c|c|}
\hline \multirow[b]{2}{*}{$\rho$} & \multirow[b]{2}{*}{$\hat{\rho}$} & \multirow[b]{2}{*}{ RMSE } & \multicolumn{3}{|c|}{ Coverage probabilities } & \multirow[b]{2}{*}{$|\overline{\hat{D}}|$} & \multirow[b]{2}{*}{$|\overline{\hat{G}}|$} & \multirow[b]{2}{*}{$\frac{|\overline{\hat{G}}|}{|\hat{\hat{D}}|}$} \\
\hline & & & Cov-1 & Cov-2 & Cov-3 & & & \\
\hline & \multicolumn{8}{|c|}{$n=50$} \\
\hline 0.20 & 0.928 & 6.203 & 67.88 & 83.98 & 83.99 & 0.643 & 89.291 & 138.89 \\
\hline 0.30 & 0.756 & 2.566 & 73.45 & 87.77 & 87.74 & 0.561 & 34.497 & 61.49 \\
\hline 0.50 & 0.730 & 1.160 & 79.34 & 92.31 & 92.30 & 0.428 & 30.346 & 70.86 \\
\hline 0.75 & 0.909 & 0.698 & 78.29 & 91.31 & 91.25 & 0.299 & 4.937 & 16.51 \\
\hline 0.80 & 0.966 & 1.042 & 78.21 & 90.44 & 90.37 & 0.269 & 2.813 & 10.46 \\
\hline 0.90 & 1.044 & 0.482 & 78.34 & 88.84 & 88.83 & 0.198 & 3.010 & 15.20 \\
\hline 0.95 & 1.079 & 0.540 & 78.46 & 87.69 & 87.66 & 0.137 & 2.832 & 20.67 \\
\hline 0.97 & 1.097 & 0.369 & 78.59 & 87.19 & 87.16 & 0.117 & 2.908 & 24.85 \\
\hline 0.98 & 1.104 & 0.359 & 78.78 & 86.87 & 86.86 & 0.105 & 2.911 & 27.72 \\
\hline 1.00 & 1.119 & 0.363 & 78.78 & 86.16 & 86.16 & 0.089 & 2.997 & 33.67 \\
\hline 1.10 & 1.197 & 0.355 & 79.35 & 83.12 & 83.07 & 0.118 & 3.322 & 28.15 \\
\hline 1.20 & 1.277 & 0.356 & 79.28 & 79.66 & 79.50 & 0.241 & 3.558 & 14.76 \\
\hline 1.30 & 1.361 & 0.349 & 79.21 & 77.65 & 77.55 & 0.366 & 3.851 & 10.52 \\
\hline \multirow[t]{2}{*}{1.50} & 1.546 & 0.313 & 81.57 & 78.08 & 78.03 & 0.544 & 4.385 & 8.06 \\
\hline & \multicolumn{8}{|c|}{$n=100$} \\
\hline 0.20 & 0.503 & 2.157 & 72.82 & 87.82 & 87.80 & 0.663 & 21.984 & 33.13 \\
\hline 0.30 & 0.502 & 4.404 & 79.12 & 91.97 & 91.97 & 0.617 & 96.243 & 155.90 \\
\hline 0.50 & 0.618 & 0.504 & 82.48 & 95.27 & 95.24 & 0.416 & 22.801 & 54.78 \\
\hline 0.75 & 0.884 & 0.336 & 80.52 & 93.77 & 93.76 & 0.293 & 2.788 & 9.52 \\
\hline 0.80 & 0.931 & 0.325 & 80.30 & 93.31 & 93.33 & 0.267 & 2.248 & 8.42 \\
\hline 0.90 & 1.015 & 0.293 & 80.44 & 91.78 & 91.78 & 0.180 & 2.333 & 12.96 \\
\hline 0.95 & 1.047 & 0.291 & 80.56 & 90.30 & 90.30 & 0.115 & 2.539 & 22.08 \\
\hline 0.97 & 1.059 & 0.289 & 80.66 & 89.70 & 89.67 & 0.088 & 2.592 & 29.45 \\
\hline 0.98 & 1.066 & 0.289 & 80.72 & 89.44 & 89.39 & 0.078 & 2.626 & 33.67 \\
\hline 1.00 & 1.078 & 0.289 & 80.63 & 88.54 & 88.49 & 0.062 & 2.738 & 44.16 \\
\hline 1.10 & 1.160 & 0.296 & 81.17 & 84.86 & 84.73 & 0.131 & 3.066 & 23.40 \\
\hline 1.20 & 1.242 & 0.297 & 80.87 & 81.22 & 81.16 & 0.265 & 3.402 & 12.84 \\
\hline 1.30 & 1.330 & 0.288 & 81.37 & 80.15 & 80.07 & 0.382 & 3.702 & 9.69 \\
\hline \multirow[t]{2}{*}{1.50} & 1.531 & 0.245 & 84.99 & 83.83 & 83.79 & 0.515 & 4.230 & 8.21 \\
\hline & \multicolumn{8}{|c|}{$n=200$} \\
\hline 0.20 & 0.334 & 1.149 & 80.68 & 93.16 & 93.16 & 0.669 & 5.359 & 8.01 \\
\hline 0.30 & 0.381 & 0.462 & 86.09 & 96.62 & 96.59 & 0.567 & 95.114 & 167.85 \\
\hline 0.50 & 0.574 & 0.226 & 85.87 & 96.99 & 97.00 & 0.438 & 4.809 & 10.97 \\
\hline 0.75 & 0.844 & 0.248 & 81.51 & 94.70 & 94.71 & 0.275 & 2.044 & 7.43 \\
\hline 0.80 & 0.897 & 0.250 & 81.60 & 94.51 & 94.44 & 0.248 & 2.025 & 8.17 \\
\hline 0.90 & 0.989 & 0.246 & 81.08 & 93.39 & 93.40 & 0.168 & 2.171 & 12.92 \\
\hline 0.95 & 1.022 & 0.244 & 80.93 & 92.19 & 92.14 & 0.101 & 2.351 & 23.28 \\
\hline 0.97 & 1.034 & 0.242 & 81.11 & 91.36 & 91.34 & 0.072 & 2.428 & 33.72 \\
\hline 0.98 & 1.039 & 0.241 & 81.25 & 90.99 & 90.99 & 0.058 & 2.479 & 42.74 \\
\hline 1.00 & 1.051 & 0.241 & 81.45 & 90.02 & 89.97 & 0.044 & 2.574 & 58.50 \\
\hline 1.10 & 1.125 & 0.250 & 81.49 & 84.85 & 84.76 & 0.169 & 2.998 & 17.74 \\
\hline 1.20 & 1.211 & 0.250 & 81.17 & 81.71 & 81.65 & 0.300 & 3.336 & 11.12 \\
\hline 1.30 & 1.316 & 0.235 & 82.78 & 83.10 & 83.05 & 0.361 & 3.554 & 9.84 \\
\hline 1.50 & 1.527 & 0.184 & 88.21 & 90.25 & 90.20 & 0.471 & 4.103 & 8.71 \\
\hline
\end{tabular}

Continued over 
Table 1 (continued): GMM estimation of panel data model

\begin{tabular}{|c|c|c|c|c|c|c|c|c|}
\hline \multirow[b]{2}{*}{$\rho$} & \multirow[b]{2}{*}{$\hat{\rho}$} & \multirow[b]{2}{*}{ RMSE } & \multicolumn{3}{|c|}{ Coverage probabilities } & \multirow[b]{2}{*}{$|\overline{\hat{D}}|$} & \multirow[b]{2}{*}{$|\overline{\hat{G}}|$} & \multirow[b]{2}{*}{$\frac{|\overline{\hat{G}}|}{|\hat{D}|}$} \\
\hline & & & Cov-1 & Cov-2 & Cov-3 & & & \\
\hline & \multicolumn{8}{|c|}{$n=1000$} \\
\hline 0.20 & 0.226 & 0.069 & 91.07 & 99.63 & 99.63 & 0.724 & 2.015 & 2.78 \\
\hline 0.30 & 0.320 & 0.070 & 92.98 & 99.94 & 99.94 & 0.657 & 1.191 & 1.81 \\
\hline 0.50 & 0.521 & 0.096 & 91.68 & 99.82 & 99.82 & 0.484 & 1.156 & 2.39 \\
\hline 0.75 & 0.784 & 0.154 & 83.61 & 96.78 & 96.72 & 0.253 & 1.718 & 6.79 \\
\hline 0.80 & 0.839 & 0.163 & 82.15 & 96.24 & 96.17 & 0.215 & 1.815 & 8.44 \\
\hline 0.90 & 0.961 & 0.166 & 81.96 & 97.11 & 97.08 & 0.168 & 1.928 & 11.48 \\
\hline 0.95 & 1.005 & 0.162 & 81.91 & 96.18 & 96.11 & 0.118 & 2.076 & 17.59 \\
\hline 0.97 & 1.014 & 0.160 & 82.22 & 95.35 & 95.28 & 0.079 & 2.177 & 27.56 \\
\hline 0.98 & 1.019 & 0.160 & 82.13 & 94.96 & 94.93 & 0.060 & 2.227 & 37.12 \\
\hline 1.00 & 1.025 & 0.159 & 82.52 & 93.77 & 93.74 & 0.019 & 2.351 & 123.74 \\
\hline 1.10 & 1.092 & 0.168 & 83.07 & 87.35 & 87.23 & 0.174 & 2.818 & 16.20 \\
\hline 1.20 & 1.198 & 0.158 & 83.28 & 87.18 & 87.12 & 0.251 & 3.079 & 12.27 \\
\hline 1.30 & 1.312 & 0.129 & 88.66 & 93.32 & 93.23 & 0.293 & 3.308 & 11.29 \\
\hline \multirow[t]{2}{*}{1.50} & 1.512 & 0.088 & 93.03 & 98.47 & 98.46 & 0.470 & 4.028 & 8.57 \\
\hline & \multicolumn{8}{|c|}{$n=5000$} \\
\hline 0.20 & 0.204 & 0.026 & 94.27 & 100.00 & 100.00 & 0.787 & 0.616 & 0.78 \\
\hline 0.30 & 0.302 & 0.029 & 94.52 & 100.00 & 100.00 & 0.692 & 0.623 & 0.90 \\
\hline 0.50 & 0.503 & 0.042 & 93.67 & 100.00 & 100.00 & 0.496 & 0.950 & 1.92 \\
\hline 0.75 & 0.760 & 0.083 & 87.93 & 99.29 & 99.29 & 0.250 & 1.565 & 6.26 \\
\hline 0.80 & 0.812 & 0.096 & 84.66 & 98.35 & 98.35 & 0.202 & 1.710 & 8.47 \\
\hline 0.90 & 0.927 & 0.114 & 81.06 & 97.43 & 97.41 & 0.127 & 1.943 & 15.30 \\
\hline 0.95 & 0.992 & 0.113 & 81.72 & 97.74 & 97.71 & 0.113 & 2.013 & 17.81 \\
\hline 0.97 & 1.009 & 0.111 & 81.59 & 97.02 & 97.00 & 0.089 & 2.083 & 23.40 \\
\hline 0.98 & 1.013 & 0.110 & 81.51 & 96.53 & 96.49 & 0.069 & 2.134 & 30.93 \\
\hline 1.00 & 1.016 & 0.108 & 82.17 & 94.87 & 94.84 & 0.014 & 2.268 & 162.00 \\
\hline 1.10 & 1.084 & 0.115 & 81.18 & 87.80 & 87.77 & 0.164 & 2.726 & 16.62 \\
\hline 1.20 & 1.207 & 0.089 & 87.69 & 95.47 & 95.43 & 0.194 & 2.922 & 15.06 \\
\hline 1.30 & 1.308 & 0.065 & 92.14 & 99.23 & 99.20 & 0.285 & 3.255 & 11.42 \\
\hline 1.50 & 1.504 & 0.040 & 94.20 & 99.98 & 99.98 & 0.488 & 4.031 & 8.26 \\
\hline
\end{tabular}

Notes: Simulated mean and root-mean-squared-error of the GMM estimator of $\rho$; coverage probability of confidence intervals (in \%) based on: the standard asymptotic theory assuming first-order local identification (Cov-1); the asymptotic distribution in Theorem 1(b), using asymptotic critical values (Cov-2) and simulated critical values $(\mathrm{Cov}-3) ;|\overline{\hat{D}}|$ is the norm of the simulated mean of the Jacobian; $|\overline{\hat{G}}|$ is the norm of the second-order derivative of the moment function. The true underlying data set has a dynamic panel structure $($ Example 1$)$ with $\sigma_{0}^{2}=\sigma_{\varepsilon}^{2}=1$, $\sigma_{\eta}^{2}=\sigma_{0 \eta}=0$ and $\rho$ as in the table. (10,000 runs) 

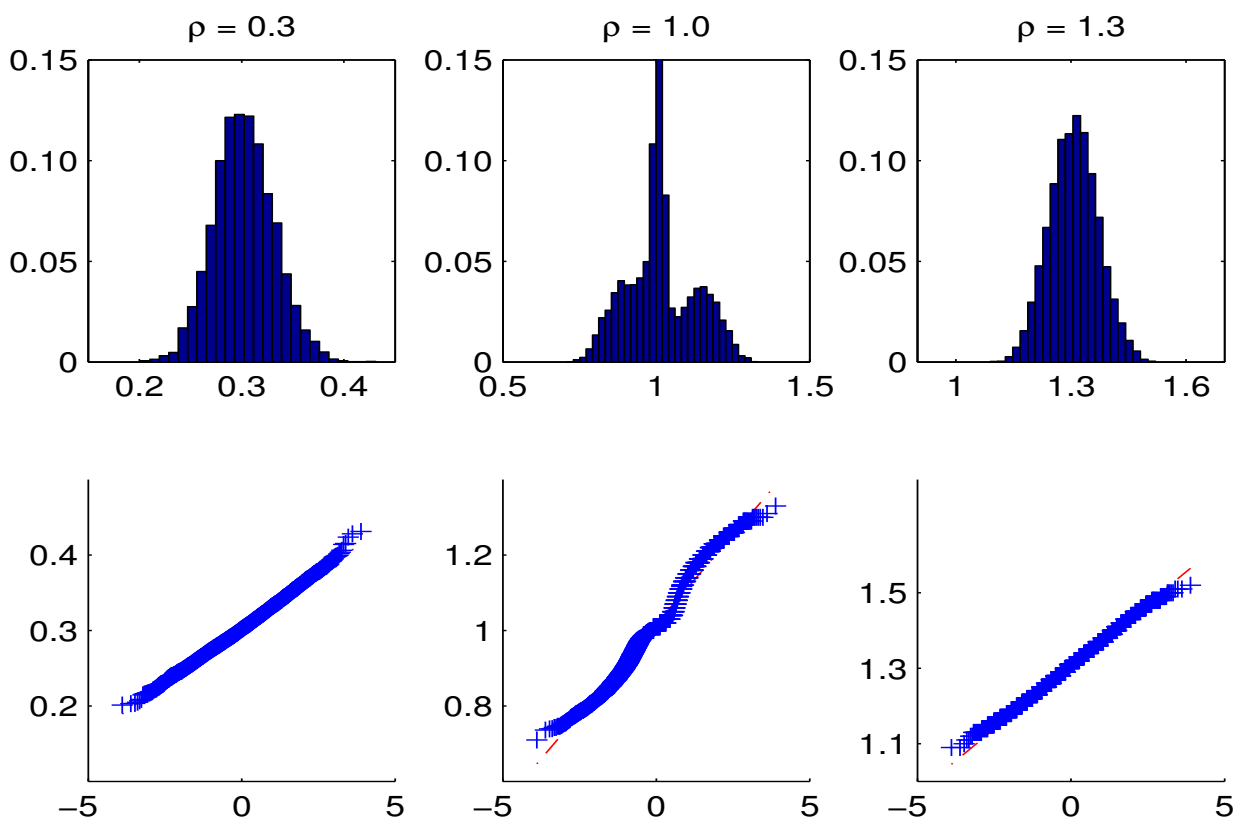

Figure 1: Histogram of simulated GMM estimator of $\rho ; \rho=0.3,1.0$ and 1.3, respectively and their QQ-plot versus the standard normal distribution. The true underlying data set has a dynamic panel structure (Example 1) with $\sigma_{0}^{2}=\sigma_{\varepsilon}^{2}=1, \sigma_{\eta}^{2}=\sigma_{0 \eta}=0$ and $\rho$. Simulated sample size $n=5,000$. (10,000 runs) 
Table 2: GMM estimation of panel data model

\begin{tabular}{|c|c|c|c|c|c|c|c|c|}
\hline \multirow[b]{2}{*}{$\lambda$} & \multirow[b]{2}{*}{$\hat{\rho}$} & \multirow[b]{2}{*}{ RMSE } & \multicolumn{3}{|c|}{ Coverage probabilities } & \multirow[b]{2}{*}{$|\overline{\hat{D}}|$} & \multirow[b]{2}{*}{$|\overline{\hat{G}}|$} & \multirow[b]{2}{*}{$\frac{|\overline{\hat{G}}|}{|\hat{D}|}$} \\
\hline & & & Cov-1 & Cov-2 & Cov-3 & & & \\
\hline & \multicolumn{8}{|c|}{$n=50$} \\
\hline-0.50 & 1.131 & 0.439 & 78.96 & 89.25 & 89.23 & 0.694 & 3.019 & 4.35 \\
\hline-0.30 & 1.154 & 0.447 & 76.48 & 85.32 & 85.29 & 0.410 & 3.037 & 7.41 \\
\hline-0.20 & 1.152 & 0.397 & 76.55 & 84.91 & 84.87 & 0.279 & 2.994 & 10.73 \\
\hline-0.10 & 1.138 & 0.387 & 77.72 & 85.25 & 85.20 & 0.164 & 2.960 & 18.05 \\
\hline 0.00 & 1.119 & 0.363 & 78.78 & 86.16 & 86.16 & 0.089 & 2.997 & 33.67 \\
\hline 0.10 & 1.109 & 0.373 & 79.25 & 80.08 & 80.01 & 0.386 & 4.352 & 11.27 \\
\hline 0.20 & 1.095 & 0.384 & 79.20 & 74.45 & 74.35 & 0.787 & 5.688 & 7.23 \\
\hline 0.30 & 1.084 & 0.382 & 78.75 & 70.64 & 70.57 & 1.172 & 6.955 & 5.93 \\
\hline \multirow[t]{2}{*}{0.50} & 1.067 & 0.350 & 79.39 & 69.56 & 69.54 & 1.826 & 8.937 & 4.89 \\
\hline & \multicolumn{8}{|c|}{$n=100$} \\
\hline-0.50 & 1.105 & 0.406 & 82.62 & 93.77 & 93.71 & 0.652 & 2.663 & 4.08 \\
\hline-0.30 & 1.139 & 0.346 & 77.56 & 88.78 & 88.77 & 0.344 & 2.594 & 7.54 \\
\hline-0.20 & 1.139 & 0.338 & 76.91 & 87.15 & 87.01 & 0.220 & 2.599 & 11.81 \\
\hline-0.10 & 1.115 & 0.314 & 78.23 & 87.51 & 87.41 & 0.128 & 2.633 & 20.57 \\
\hline 0.00 & 1.078 & 0.289 & 80.63 & 88.54 & 88.49 & 0.062 & 2.738 & 44.16 \\
\hline 0.10 & 1.064 & 0.311 & 80.92 & 80.98 & 80.92 & 0.407 & 4.036 & 9.92 \\
\hline 0.20 & 1.050 & 0.317 & 81.23 & 75.90 & 75.84 & 0.804 & 5.297 & 6.59 \\
\hline 0.30 & 1.052 & 0.313 & 80.92 & 73.66 & 73.56 & 1.113 & 6.278 & 5.64 \\
\hline \multirow[t]{2}{*}{0.50} & 1.052 & 0.275 & 82.41 & 75.82 & 75.66 & 1.657 & 7.919 & 4.78 \\
\hline & \multicolumn{8}{|c|}{$n=200$} \\
\hline-0.50 & 1.069 & 0.227 & 86.54 & 97.30 & 97.25 & 0.657 & 2.545 & 3.87 \\
\hline-0.30 & 1.114 & 0.284 & 79.79 & 91.96 & 91.86 & 0.324 & 2.407 & 7.43 \\
\hline-0.20 & 1.122 & 0.293 & 77.01 & 89.05 & 89.00 & 0.190 & 2.399 & 12.63 \\
\hline-0.10 & 1.101 & 0.272 & 77.49 & 88.97 & 88.93 & 0.101 & 2.437 & 24.13 \\
\hline 0.00 & 1.051 & 0.241 & 81.45 & 90.02 & 89.97 & 0.044 & 2.574 & 58.50 \\
\hline 0.10 & 1.027 & 0.258 & 82.13 & 81.93 & 81.86 & 0.436 & 3.880 & 8.90 \\
\hline 0.20 & 1.031 & 0.264 & 82.19 & 78.19 & 78.21 & 0.755 & 4.901 & 6.49 \\
\hline 0.30 & 1.042 & 0.255 & 81.73 & 77.51 & 77.49 & 1.007 & 5.715 & 5.68 \\
\hline \multirow[t]{2}{*}{0.50} & 1.044 & 0.209 & 85.35 & 82.39 & 82.39 & 1.525 & 7.268 & 4.77 \\
\hline & \multicolumn{8}{|c|}{$n=1000$} \\
\hline-0.50 & 1.014 & 0.087 & 94.20 & 99.92 & 99.94 & 0.694 & 2.496 & 3.60 \\
\hline-0.30 & 1.047 & 0.152 & 88.35 & 97.22 & 97.24 & 0.365 & 2.322 & 6.36 \\
\hline-0.20 & 1.073 & 0.188 & 82.07 & 94.02 & 93.96 & 0.192 & 2.235 & 11.64 \\
\hline-0.10 & 1.077 & 0.196 & 77.61 & 91.66 & 91.61 & 0.070 & 2.220 & 31.71 \\
\hline 0.00 & 1.025 & 0.159 & 82.52 & 93.77 & 93.74 & 0.019 & 2.351 & 123.74 \\
\hline 0.10 & 1.004 & 0.169 & 85.25 & 87.35 & 87.23 & 0.384 & 3.481 & 9.07 \\
\hline 0.20 & 1.031 & 0.168 & 85.00 & 86.69 & 86.62 & 0.597 & 4.220 & 7.07 \\
\hline 0.30 & 1.031 & 0.145 & 86.89 & 89.10 & 88.97 & 0.878 & 5.063 & 5.77 \\
\hline \multirow[t]{2}{*}{0.50} & 1.016 & 0.090 & 93.12 & 96.85 & 96.79 & 1.517 & 6.894 & 4.54 \\
\hline & \multicolumn{8}{|c|}{$n=5000$} \\
\hline-0.50 & 1.002 & 0.035 & 94.86 & 100.00 & 100.00 & 0.707 & 2.504 & 3.54 \\
\hline-0.30 & 1.008 & 0.057 & 94.77 & 99.90 & 99.89 & 0.415 & 2.375 & 5.72 \\
\hline-0.20 & 1.024 & 0.096 & 89.69 & 97.18 & 97.14 & 0.248 & 2.287 & 9.22 \\
\hline-0.10 & 1.052 & 0.137 & 78.20 & 91.44 & 91.50 & 0.072 & 2.185 & 30.35 \\
\hline 0.00 & 1.016 & 0.108 & 82.17 & 94.87 & 94.84 & 0.014 & 2.268 & 162.00 \\
\hline 0.10 & 1.016 & 0.116 & 84.14 & 90.46 & 90.27 & 0.300 & 3.216 & 10.72 \\
\hline 0.20 & 1.018 & 0.094 & 88.13 & 92.52 & 92.50 & 0.588 & 4.083 & 6.94 \\
\hline 0.30 & 1.008 & 0.060 & 93.40 & 98.37 & 98.36 & 0.927 & 5.045 & 5.44 \\
\hline 0.50 & 1.003 & 0.037 & 93.76 & 99.89 & 99.89 & 1.569 & 6.936 & 4.42 \\
\hline
\end{tabular}

Notes: Definitions as Table 1 except that the true underlying data set has a dynamic panel structure (Example 1) with $\rho=1, \sigma_{0}^{2}=\sigma_{\varepsilon}^{2}=1, \sigma_{0 \eta}=\lambda, \sigma_{\eta}^{2}=|\lambda|$, with $\lambda$ as in the table. 
Table 3: GMM and II estimation of panel data model

\begin{tabular}{|c|c|c|c|c|c|c|c|c|c|}
\hline \multirow[b]{2}{*}{$\rho$} & \multicolumn{2}{|c|}{ Bias } & \multicolumn{2}{|c|}{ RMSE } & \multicolumn{2}{|c|}{ Cov. probability } & \multirow[b]{2}{*}{$|\overline{\hat{D}}|$} & \multirow[b]{2}{*}{$|\overline{\hat{G}}|$} & \multirow[b]{2}{*}{$\frac{|\overline{\hat{G}}|}{|\hat{D}|}$} \\
\hline & GMM & II & GMM & II & Cov-1ii & Cov-2ii & & & \\
\hline & & & & & $n=50$ & & & & \\
\hline 0.30 & 0.486 & 0.349 & 2.402 & 2.383 & 82.70 & 87.50 & 0.511 & 1.656 & 3.240 \\
\hline 0.80 & 0.140 & -0.035 & 0.548 & 0.511 & 83.50 & 89.96 & 0.249 & 3.442 & 13.806 \\
\hline 0.90 & 0.131 & -0.054 & 0.483 & 0.471 & 75.78 & 78.12 & 0.469 & 4.561 & 9.729 \\
\hline 1.00 & 0.101 & -0.088 & 0.366 & 0.388 & 67.12 & 67.94 & 0.802 & 5.909 & 7.370 \\
\hline 1.20 & 0.073 & -0.105 & 0.355 & 0.426 & 67.18 & 66.26 & 1.451 & 8.179 & 5.638 \\
\hline \multirow[t]{2}{*}{1.30} & 0.063 & -0.109 & 0.348 & 0.432 & 66.06 & 63.28 & 1.678 & 8.719 & 5.196 \\
\hline & & & & & $n=100$ & & & & \\
\hline 0.30 & 0.118 & 0.080 & 0.611 & 0.575 & 90.40 & 97.30 & 0.653 & 1.238 & 1.896 \\
\hline 0.80 & 0.127 & 0.038 & 0.299 & 0.265 & 79.00 & 86.34 & 0.190 & 3.305 & 17.427 \\
\hline 0.90 & 0.112 & 0.017 & 0.294 & 0.262 & 80.66 & 84.38 & 0.181 & 3.369 & 18.614 \\
\hline 1.00 & 0.081 & -0.019 & 0.289 & 0.268 & 82.14 & 84.50 & 0.315 & 3.745 & 11.902 \\
\hline 1.20 & 0.039 & -0.066 & 0.298 & 0.299 & 77.92 & 78.20 & 0.703 & 4.655 & 6.622 \\
\hline \multirow[t]{2}{*}{1.30} & 0.040 & -0.062 & 0.288 & 0.294 & 79.10 & 74.42 & 0.792 & 4.787 & 6.042 \\
\hline & & & & & $n=200$ & & & & \\
\hline 0.30 & 0.079 & 0.034 & 0.403 & 0.390 & 61.30 & 71.40 & 0.558 & 3.257 & 5.839 \\
\hline 0.80 & 0.101 & 0.032 & 0.252 & 0.217 & 84.50 & 93.68 & 0.174 & 2.382 & 13.693 \\
\hline 0.90 & 0.094 & 0.019 & 0.248 & 0.216 & 84.74 & 90.18 & 0.094 & 2.510 & 26.664 \\
\hline 1.00 & 0.055 & -0.022 & 0.243 & 0.227 & 81.92 & 83.62 & 0.198 & 3.003 & 15.144 \\
\hline 1.20 & 0.006 & -0.074 & 0.251 & 0.261 & 71.60 & 70.20 & 0.597 & 3.922 & 6.575 \\
\hline \multirow[t]{2}{*}{1.30} & 0.010 & -0.069 & 0.236 & 0.252 & 75.04 & 72.54 & 0.683 & 4.103 & 6.006 \\
\hline & & & & & $n=1000$ & & & & \\
\hline 0.30 & 0.019 & -0.004 & 0.069 & 0.068 & 94.30 & 99.50 & 0.634 & 1.556 & 2.453 \\
\hline 0.80 & 0.036 & -0.004 & 0.162 & 0.149 & 85.48 & 92.04 & 0.158 & 2.025 & 12.796 \\
\hline 0.90 & 0.058 & 0.018 & 0.165 & 0.159 & 79.48 & 91.70 & 0.097 & 2.122 & 21.909 \\
\hline 1.00 & 0.021 & -0.017 & 0.158 & 0.170 & 75.66 & 85.00 & 0.107 & 2.587 & 24.218 \\
\hline 1.20 & 0.001 & -0.017 & 0.158 & 0.179 & 75.36 & 78.32 & 0.315 & 3.222 & 10.233 \\
\hline \multirow[t]{2}{*}{1.30} & 0.015 & 0.008 & 0.130 & 0.138 & 86.66 & 89.72 & 0.311 & 3.347 & 10.771 \\
\hline & & & & & $n=5000$ & & & & \\
\hline 0.30 & 0.002 & 0.002 & 0.030 & 0.030 & 94.30 & 100.00 & 0.691 & 0.645 & 0.933 \\
\hline 0.80 & 0.012 & 0.005 & 0.095 & 0.088 & 87.40 & 97.82 & 0.193 & 1.730 & 8.987 \\
\hline 0.90 & 0.026 & 0.008 & 0.112 & 0.096 & 90.12 & 96.38 & 0.098 & 1.999 & 20.426 \\
\hline 1.00 & 0.016 & -0.009 & 0.107 & 0.100 & 86.68 & 94.06 & 0.043 & 2.360 & 55.217 \\
\hline 1.20 & 0.008 & -0.016 & 0.088 & 0.098 & 82.60 & 86.48 & 0.263 & 3.033 & 11.536 \\
\hline 1.30 & 0.008 & -0.009 & 0.064 & 0.067 & 91.56 & 97.66 & 0.337 & 3.325 & 9.860 \\
\hline
\end{tabular}

Notes: Simulated mean and root-mean-squared-error of the GMM and II estimators of $\rho$; coverage probability of IIbased confidence intervals for $\rho$ using: (i) the standard II asymptotic theory assuming first-order local identification (Cov-1ii); and the result of Theorem 2 (Cov-2ii). We set $s=50$ for the estimated II binding function. For other definitions see the notes to Table 1. (5,000 runs) 

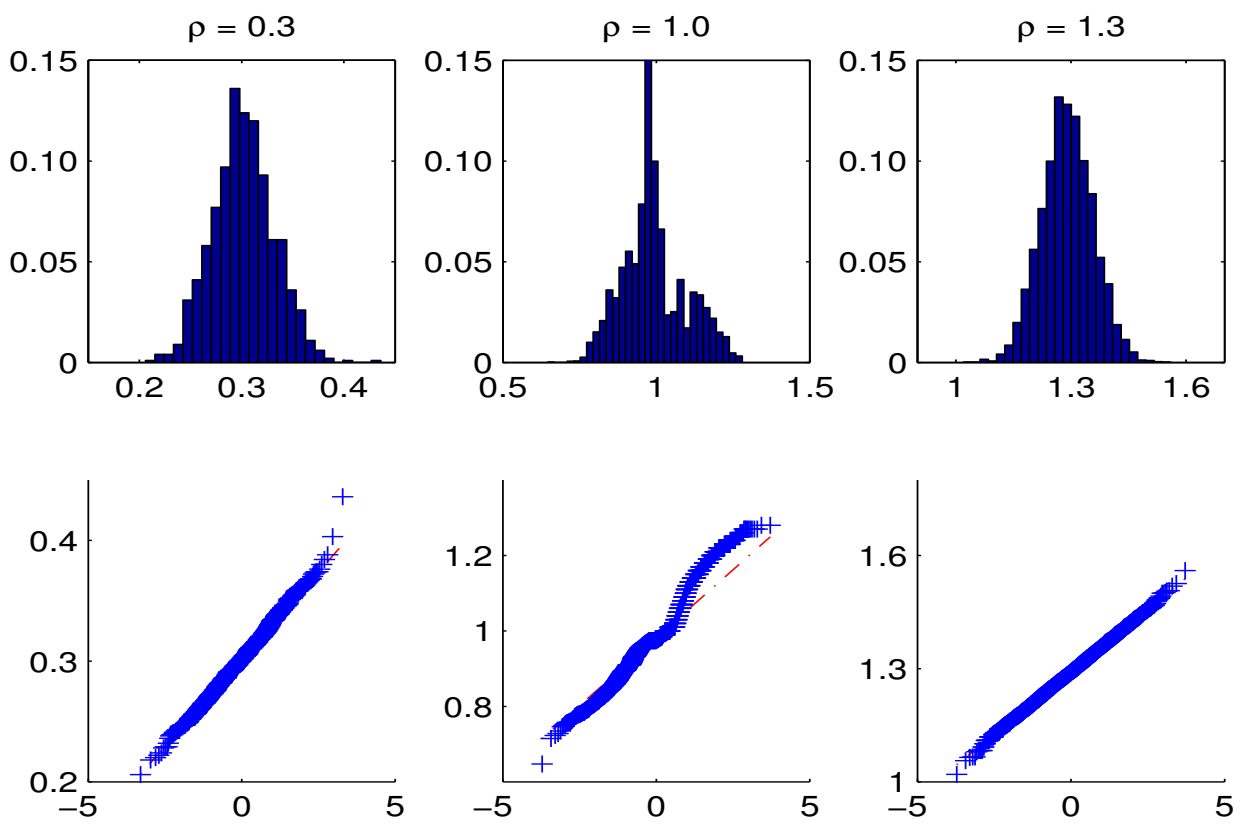

Figure 2: Histogram of simulated II estimator of $\rho ; \rho=0.3,1.0$ and 1.3, respectively and their QQplot versus the standard normal distribution. The true underlying data set has a dynamic panel structure (Example 1) with $\sigma_{0}^{2}=\sigma_{\varepsilon}^{2}=1, \sigma_{\eta}^{2}=\sigma_{0 \eta}=0$ and $\rho$. Simulated sample size $n=5,000$. (5,000 runs) 
Table 4: Robustness of II simulating model

\begin{tabular}{|c|c|c|c|c|c|c|c|c|c|}
\hline \multirow[b]{2}{*}{$\lambda$} & \multicolumn{2}{|c|}{$\overline{\text { Bias }}$} & \multicolumn{2}{|c|}{ RMSE } & \multicolumn{2}{|c|}{ Cov. probability } & \multirow[b]{2}{*}{$|\overline{\hat{D}}|$} & \multirow[b]{2}{*}{$|\overline{\hat{G}}|$} & \multirow[b]{2}{*}{$\frac{|\overline{\hat{G}}|}{|\hat{\hat{D}}|}$} \\
\hline & GMM & II & GMM & II & Cov-1ii & Cov-2ii & & & \\
\hline & \multicolumn{9}{|c|}{$n=200$} \\
\hline-0.20 & 0.051 & -0.009 & 0.244 & 0.235 & 80.10 & 83.50 & 0.171 & 2.977 & 17.436 \\
\hline-0.10 & 0.051 & -0.041 & 0.244 & 0.237 & 78.60 & 80.90 & 0.256 & 3.202 & 12.483 \\
\hline 0.00 & 0.051 & -0.025 & 0.244 & 0.229 & 80.60 & 83.10 & 0.201 & 3.018 & 15.048 \\
\hline 0.10 & 0.051 & -0.052 & 0.244 & 0.256 & 74.10 & 79.10 & 0.325 & 3.479 & 10.702 \\
\hline 0.20 & 0.051 & -0.060 & 0.244 & 0.269 & 74.60 & 81.30 & 0.401 & 3.895 & 9.724 \\
\hline \multirow[t]{2}{*}{ MAD } & & & & & 16.65 & 13.35 & & & \\
\hline & \multicolumn{9}{|c|}{$n=1000$} \\
\hline-0.20 & 0.022 & -0.022 & 0.159 & 0.176 & 73.60 & 79.80 & 0.122 & 2.625 & 21.460 \\
\hline-0.10 & 0.022 & -0.015 & 0.159 & 0.181 & 73.40 & 82.70 & 0.112 & 2.618 & 23.370 \\
\hline 0.00 & 0.022 & -0.015 & 0.159 & 0.171 & 76.10 & 85.20 & 0.104 & 2.582 & 24.801 \\
\hline 0.10 & 0.022 & 0.008 & 0.159 & 0.174 & 74.70 & 82.60 & 0.067 & 2.545 & 38.220 \\
\hline 0.20 & 0.022 & 0.008 & 0.159 & 0.160 & 87.60 & 95.90 & 0.049 & 2.473 & 50.663 \\
\hline \multirow[t]{2}{*}{ MAD } & & & & & 20.55 & 12.43 & & & \\
\hline & \multicolumn{9}{|c|}{$n=5000$} \\
\hline-0.20 & 0.019 & 0.006 & 0.107 & 0.099 & 87.00 & 95.10 & 0.011 & 2.299 & 215.015 \\
\hline-0.10 & 0.019 & 0.004 & 0.107 & 0.110 & 76.50 & 80.10 & 0.018 & 2.323 & 128.947 \\
\hline 0.00 & 0.019 & -0.006 & 0.107 & 0.100 & 87.40 & 94.90 & 0.036 & 2.351 & 65.579 \\
\hline 0.10 & 0.019 & 0.004 & 0.107 & 0.097 & 87.70 & 93.70 & 0.013 & 2.304 & 183.863 \\
\hline 0.20 & 0.019 & 0.000 & 0.107 & 0.106 & 84.90 & 94.60 & 0.025 & 2.334 & 95.251 \\
\hline MAD & & & & & 10.30 & 3.36 & & & \\
\hline
\end{tabular}

Notes: The true underlying data set has a dynamic panel structure (Example 2) with $\rho=1, \sigma_{0}^{2}=\sigma_{\varepsilon}^{2}=1$, $\sigma_{0 \eta}=\sigma_{\eta}^{2}=0$. II estimation of $\rho$ uses (12) for simulated samples and (13) as auxiliary model with $\theta_{2}$ calibrated as follows: $\tilde{\sigma}_{0}^{2}=\tilde{\sigma}_{\varepsilon}^{2}=1, \tilde{\sigma}_{0 \eta}=\lambda$ and $\tilde{\sigma}_{\eta}^{2}=|\lambda|$, with $\lambda$ as in the table. All figures in the table relate to the estimators of $\rho$. 'MAD' is the mean absolute deviation of the coverage probabilities from the nominal (95\%). For other definitions see the notes to Table 3. (1,000 runs) 